Supplementary Information for

\title{
Design of Nitroso-Modified Naphthylene-based Fluorophores as Photoactivatable Bioorthogonal Turn-on Probes
}

Yulin Tian, ${ }^{\text {a }}$ Hong Yang, ${ }^{a}$ Xiang Li, ${ }^{a}$ Yongcheng Wang, ${ }^{a}$ Yu Teng, ${ }^{a}$ and Dali Yin ${ }^{\text {a }}$

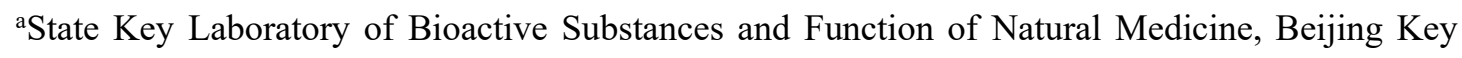
Laboratory of Active Substances Discovery and Drugability Evaluation, Institute of Materia Medica, Peking Union Medical College and Chinese Academy of Medical Sciences, 1 Xian Nong Tan Street, Beijing, 100050, China. E-mail: tianyulin@imm.ac.cn 


\section{Contents}

\section{Supplemental Figures and Tables}

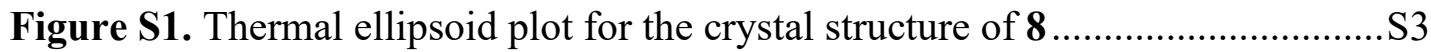

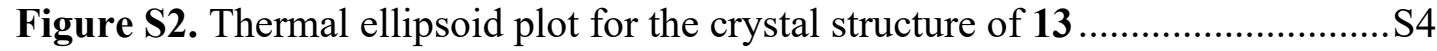

Figure S3. Thermal ellipsoid plot for the crystal structure of $14 \ldots \ldots \ldots \ldots \ldots \ldots \ldots \ldots \ldots . . .55$

Figure S4. Absorption and fluorescence emission spectra of 1, 4, 7, and 10 ..........S6

Figure S5. Absorption and fluorescence emission spectra of 2, 5, 8, and 11 ..........S6

Figure S6. Absorption and fluorescence emission spectra of 3, 6, 9, and 12 ......... S6

Figure S7. Reaction kinetic analysis for the reaction between 2 and dienes ......... S7

Figure S8. Fluorescence emission spectra of 4, 7, 10, and 14 with or without

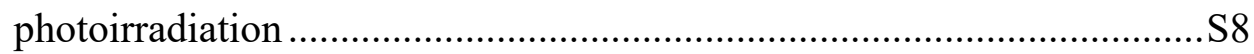

Figure S9. Photo-induced fluorescence enhancement of 5/8/11/6/9/12 ..................S9

Figure S10. HOMO-LUMO orbitals and energy diagram of 13-S .......................S10

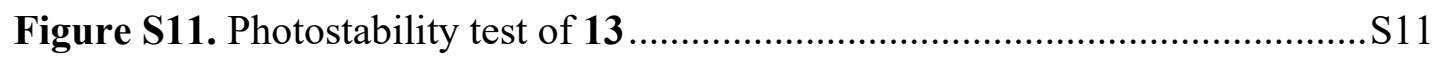

Figure S12. Fluorescence emission spectra of $\mathbf{1 3}$ in different solvent...................S1 1

Figure S13. Photo-induced fluorescence enhancement of $\mathbf{8}$ in different solvent....S12

Figure S14. Effect of Vitamin C on the formation of 13 and 15 ........................S13

Figure S15. The rate of thermally induced rearrangement from 8 to 13 ..............S13

Figure S16. In situ fluorescence measurement for the nitroso-diene reaction in cell lysates or in the presence of nucleophilic amino acids. ........................S14

Figure S17. Key ${ }^{1} \mathrm{H}-{ }^{1} \mathrm{H}$ COSY and HMBC correlations of 15 ............................S14

Figure S18. Absorption spectra of 14, 15, 16, and 17.....................................S15

Figure S19. Proposed reaction pathway for the formation of 14 and 15 ..............S15

Figure S20. MS/MS spectrum for the peptides from functionalized BSA ....... S16-17

Table S1. Photophysical properties of naphthylene probes ..................................S18

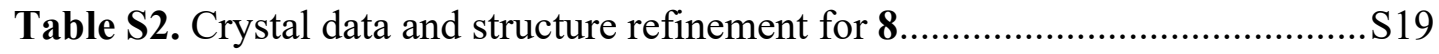

Table S3. Crystal data and structure refinement for 13....................................S20

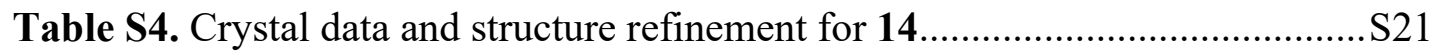

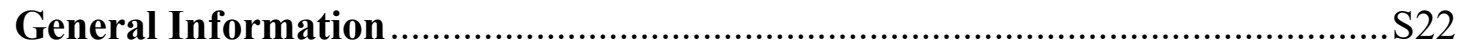

Experimental Procedures and Characterization Data ............................... S22-39

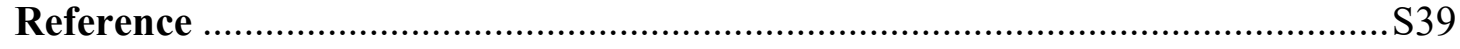

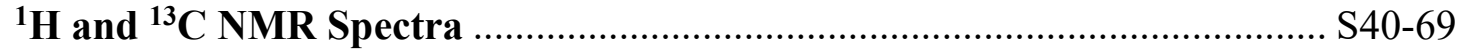




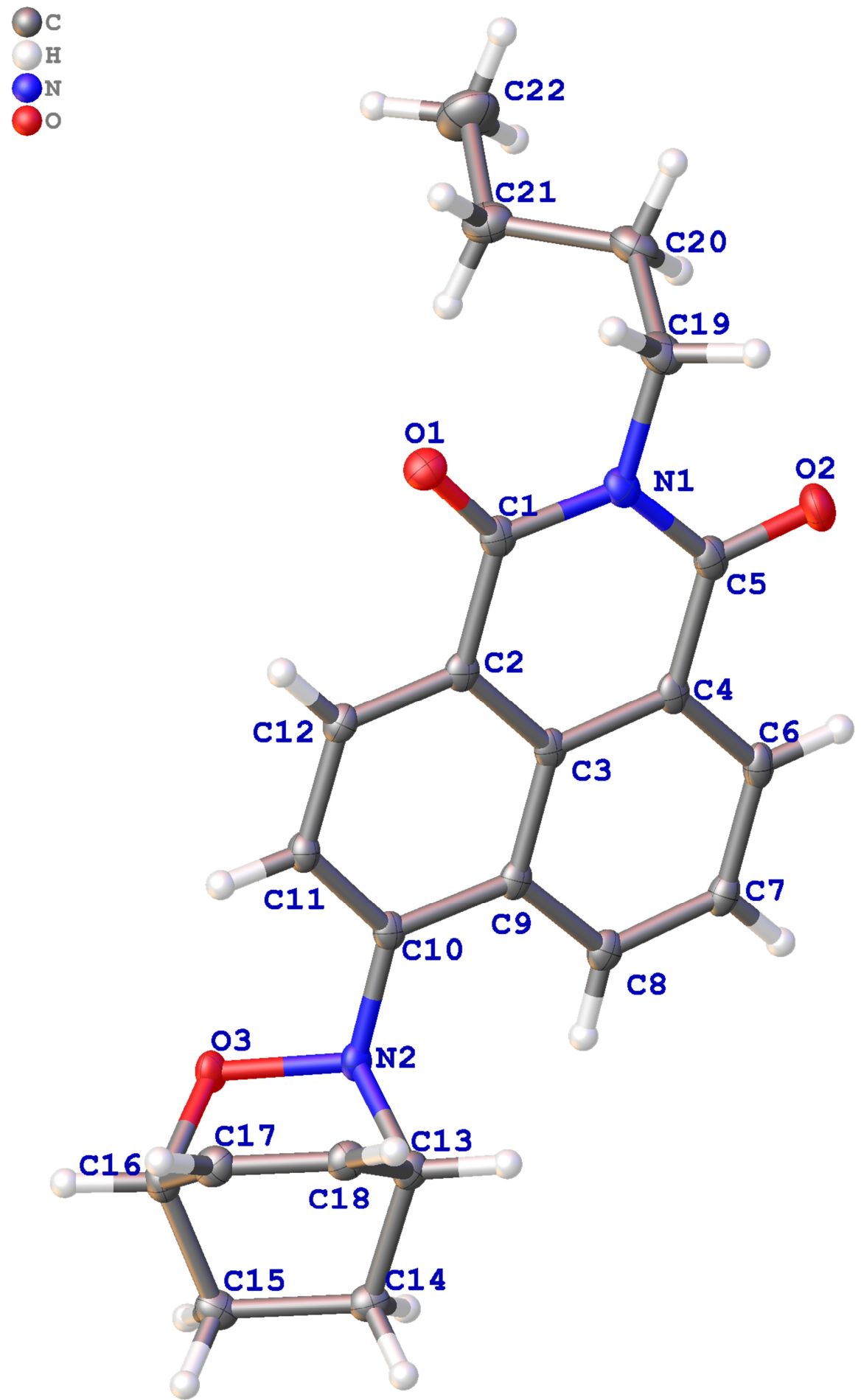

Figure S1. Thermal ellipsoid plot of the crystal structure of $\mathbf{8}(50 \%$ ellipsoid probability). 


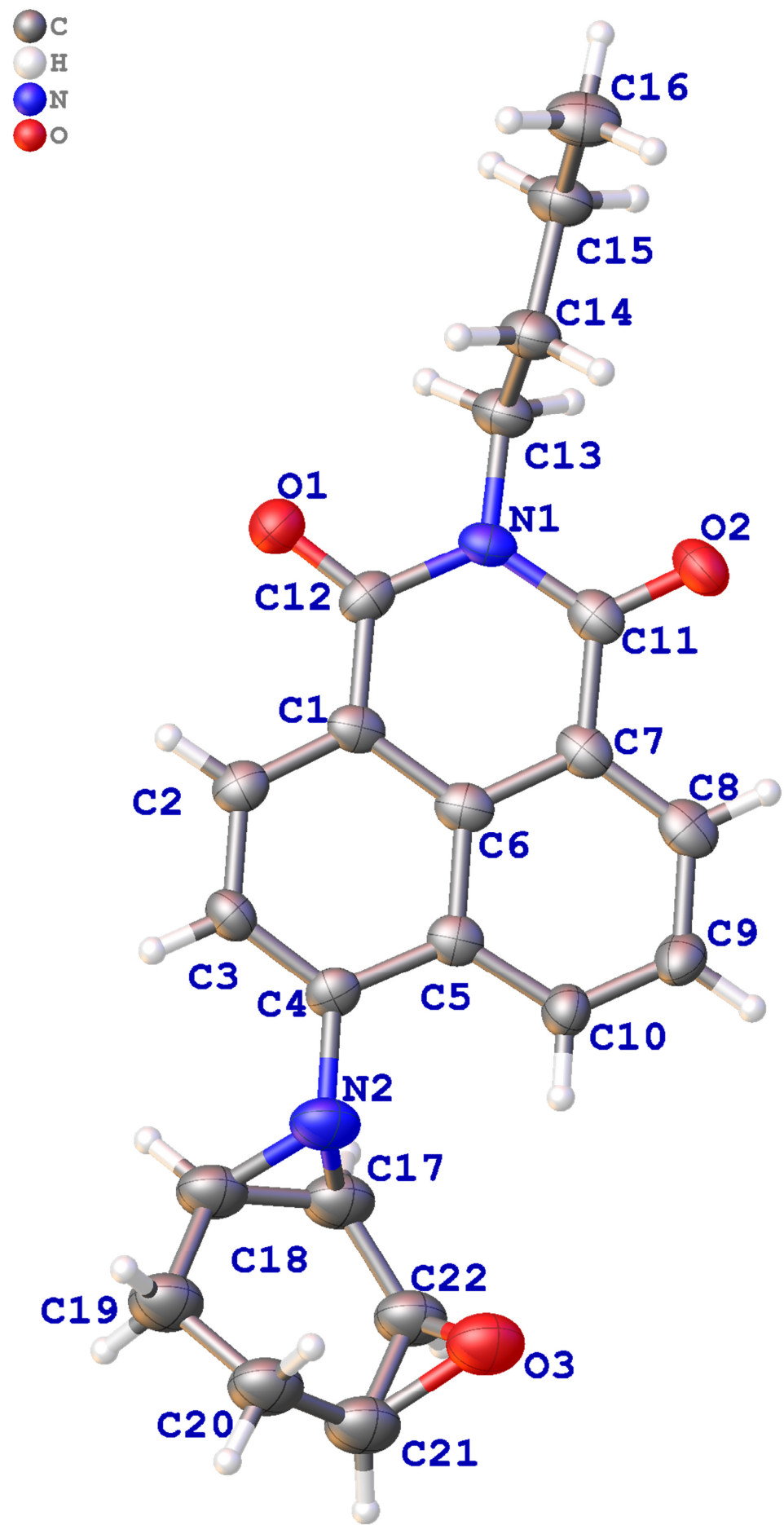

Figure S2. Thermal ellipsoid plot of the crystal structure of $\mathbf{1 3}$ (50\% ellipsoid probability). 


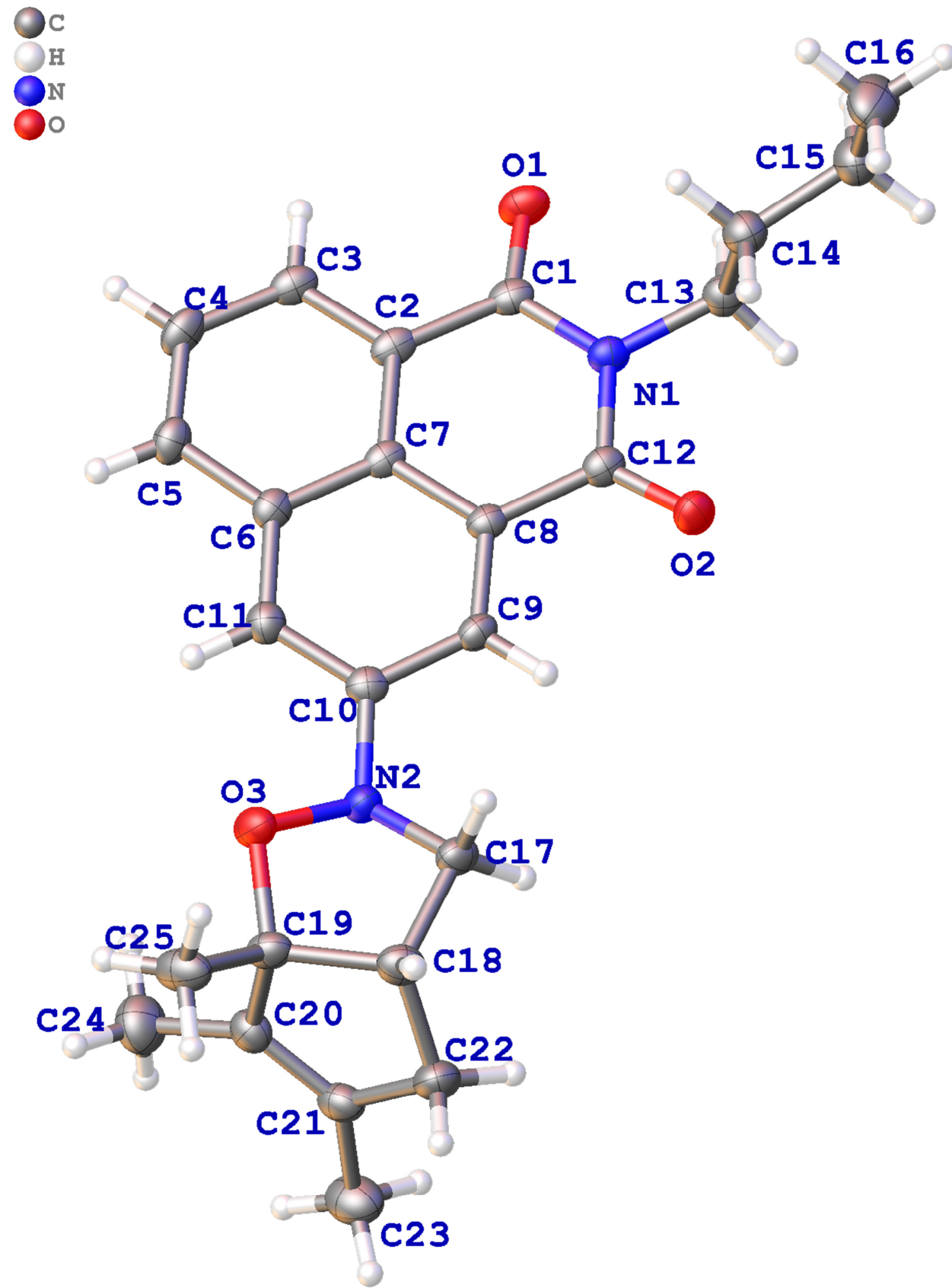

Figure S3. Thermal ellipsoid plot of the crystal structure of 14 (50\% ellipsoid probability). 
(a)

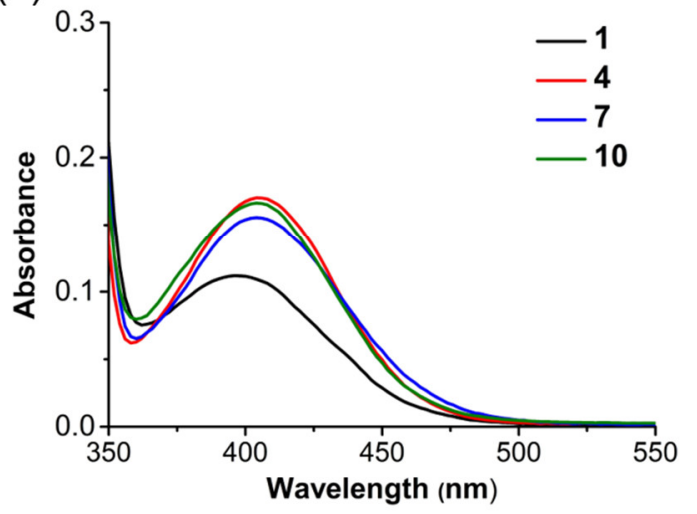

(b)

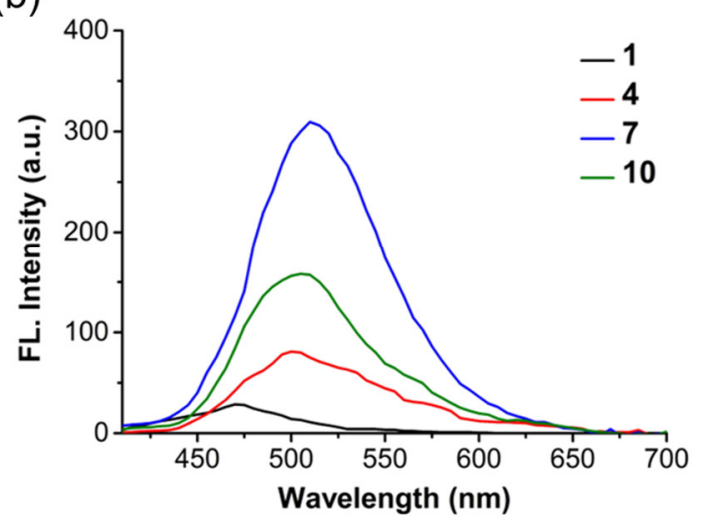

Figure S4. (a) Absorption spectra of $100 \mu \mathrm{M}$ 3-substituted-1,8-naphthalimide probes (1, 4, 7, and 10) in $\mathrm{CH}_{3} \mathrm{CN}$. (b) Fluorescence emission spectra of $10 \mu \mathrm{M}$ 3-substituted-1,8-naphthalimide probes $(\mathbf{1}, \mathbf{4}, \mathbf{7}$, and $\mathbf{1 0})$ in $\mathrm{CH}_{3} \mathrm{CN}$.

(a)

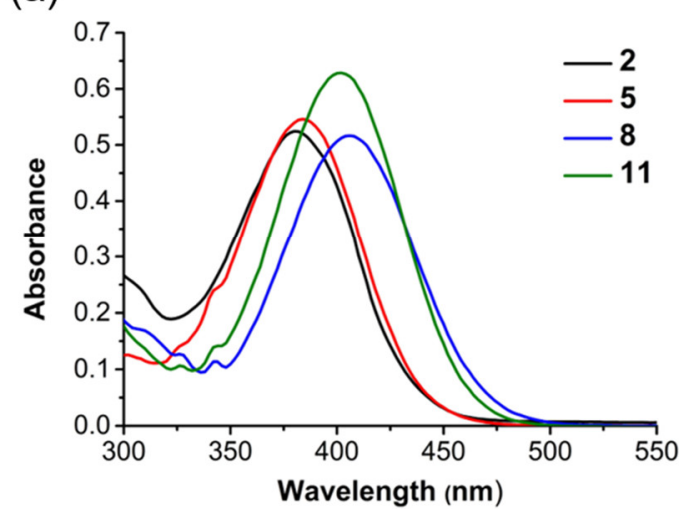

(b)

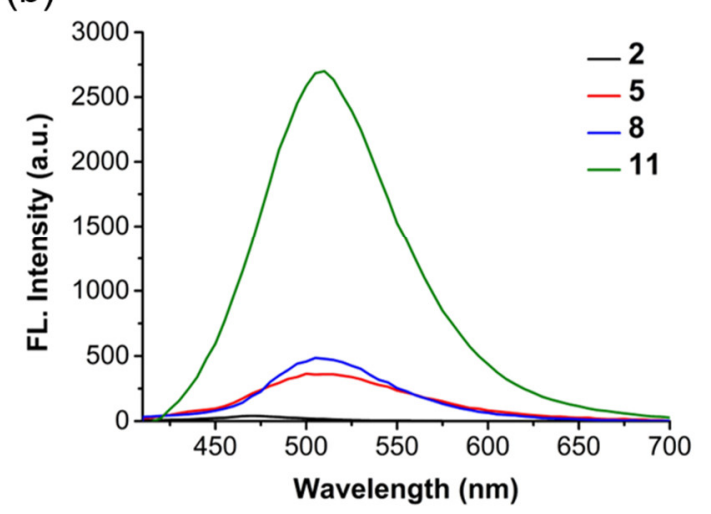

Figure S5. (a) Absorption spectra of $100 \mu \mathrm{M}$ 4-substituted-1,8-naphthalimide probes (2, 5, 8, and 11) in $\mathrm{CH}_{3} \mathrm{CN}$. (b) Fluorescence emission spectra of $10 \mu \mathrm{M}$ 4-substituted-1,8-naphthalimide probes $(\mathbf{2}, \mathbf{5}, \mathbf{8}$, and $\mathbf{1 1})$ in $\mathrm{CH}_{3} \mathrm{CN}$.

(a)

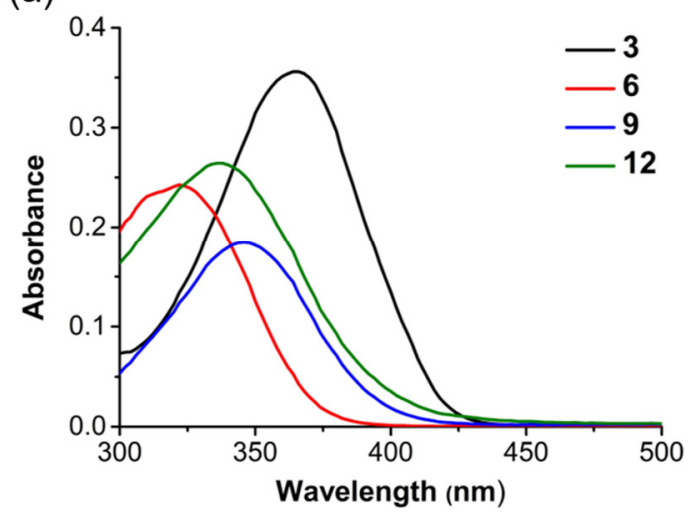

(b)

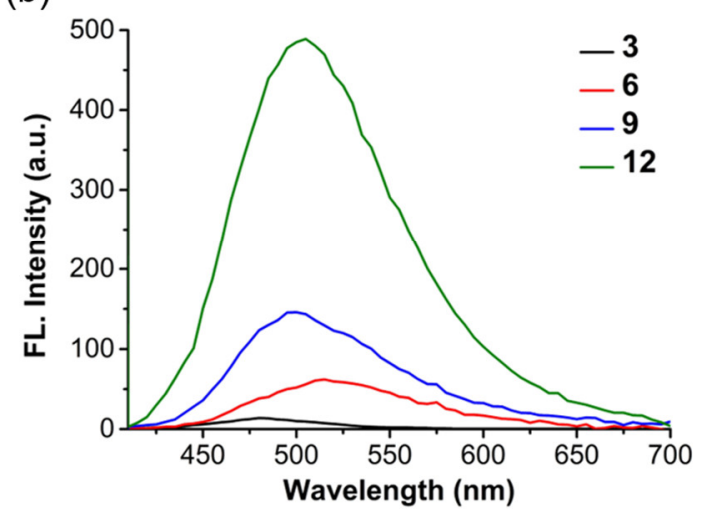

Figure S6. (a) Absorption spectra of $100 \mu \mathrm{M}$ 5-substituted-naphthalene-1sulfonamide probes $(\mathbf{3}, \mathbf{6}, \mathbf{9}$, and $\mathbf{1 2})$ in $\mathrm{CH}_{3} \mathrm{CN}$. (b) Fluorescence emission spectra of $10 \mu \mathrm{M} 5$-substituted-naphthalene-1-sulfonamide probes $(\mathbf{3}, \mathbf{6}, \mathbf{9}$, and $\mathbf{1 2})$ in $\mathrm{CH}_{3} \mathrm{CN}$. 
(a)

$$
2+\text { Cyh }
$$

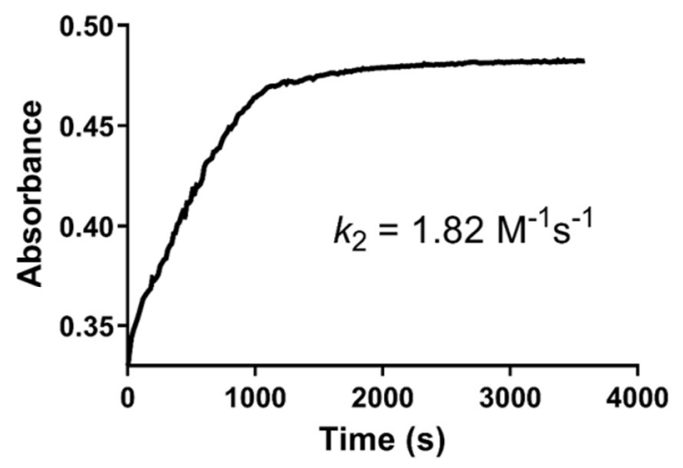

(c)

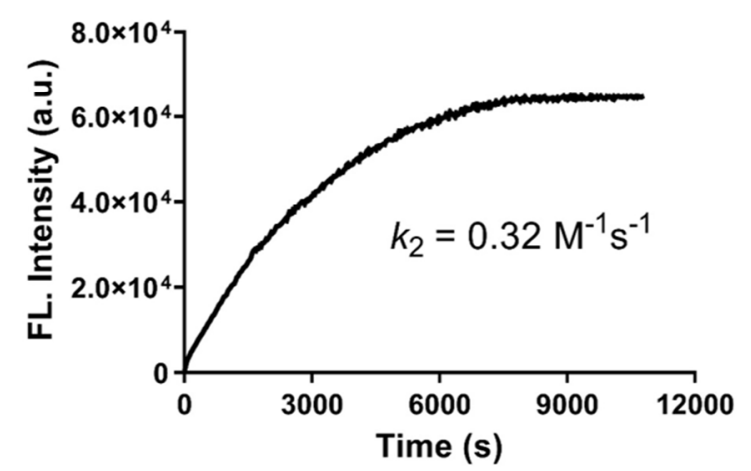

(b) $2+\mathrm{Spd}$

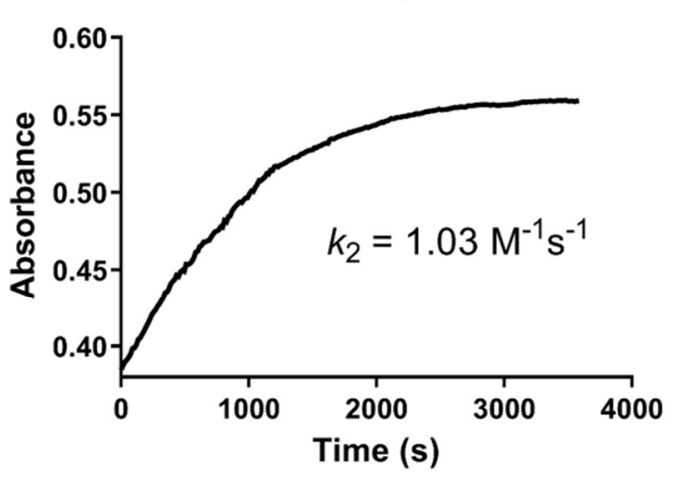

Figure S7. (a) Reaction kinetic analysis for the reaction between $2(100 \mu \mathrm{M})$ and Cyh $(1 \mathrm{mM})$ in $\mathrm{DMSO} / \mathrm{H}_{2} \mathrm{O}(4: 1, \mathrm{v} / \mathrm{v})$ at r.t. (b) Reaction kinetic analysis for the reaction between $2(100 \mu \mathrm{M})$ and $\mathrm{Spd}(1 \mathrm{mM})$ in $\mathrm{DMSO} / \mathrm{H}_{2} \mathrm{O}(4: 1, \mathrm{v} / \mathrm{v})$ at r.t. (c) Reaction kinetic analysis for the reaction between $2(100 \mu \mathrm{M})$ and $\mathrm{Mcp}(1 \mathrm{mM})$ in $\mathrm{DMSO} / \mathrm{H}_{2} \mathrm{O}$ $(4: 1, \mathrm{v} / \mathrm{v})$ at r.t. 
(a)

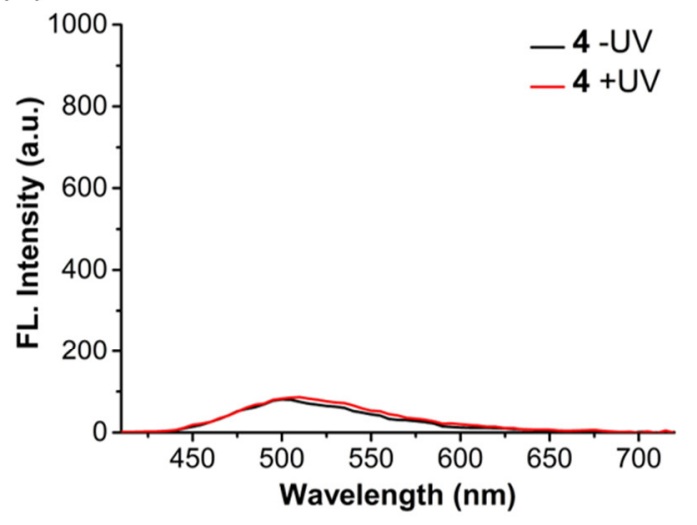

(c)

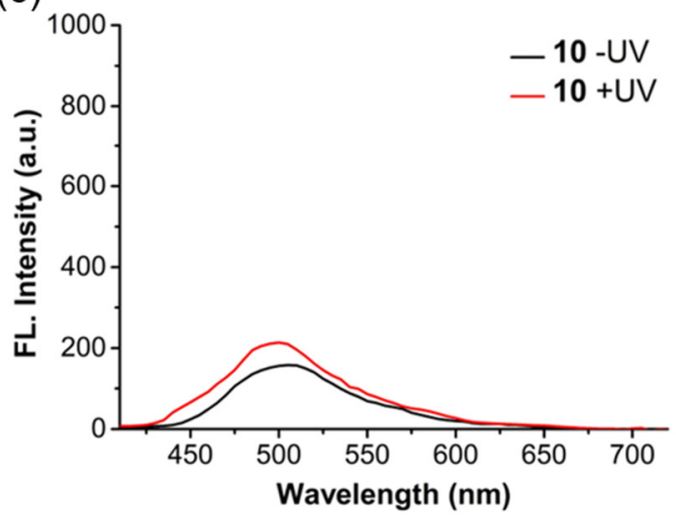

(b)

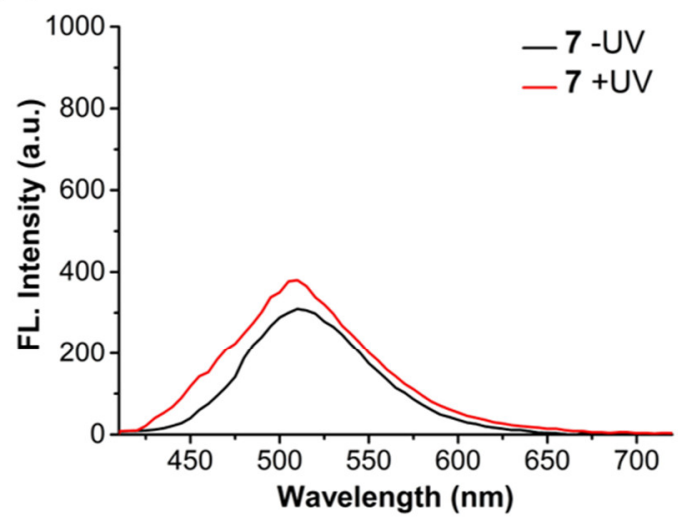

(d)

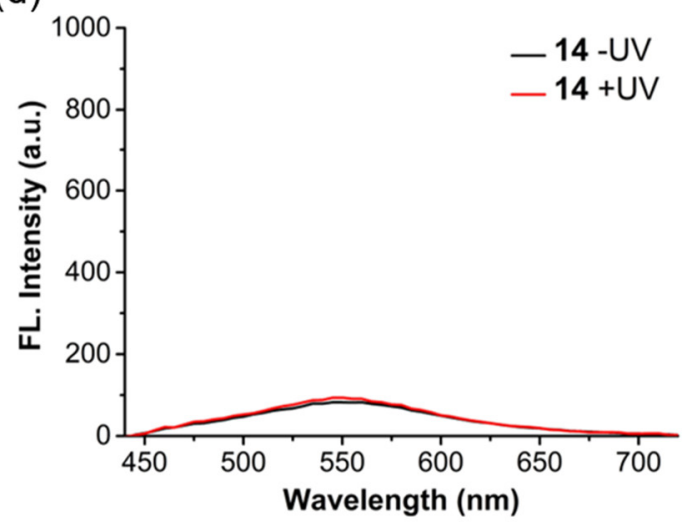

Figure S8. (a) Fluorescence emission spectra of $10 \mu \mathrm{M} 4$ (a), 7 (b), 10 (c), 14 (d) in $\mathrm{CH}_{3} \mathrm{CN}$ without photoirradiation (black) or with photoirradiation using $365 \mathrm{~nm} \mathrm{UV}$ for $60 \mathrm{~min}(\mathrm{red})\left(\lambda_{\mathrm{exc}}=365 \mathrm{~nm}\right)$. 

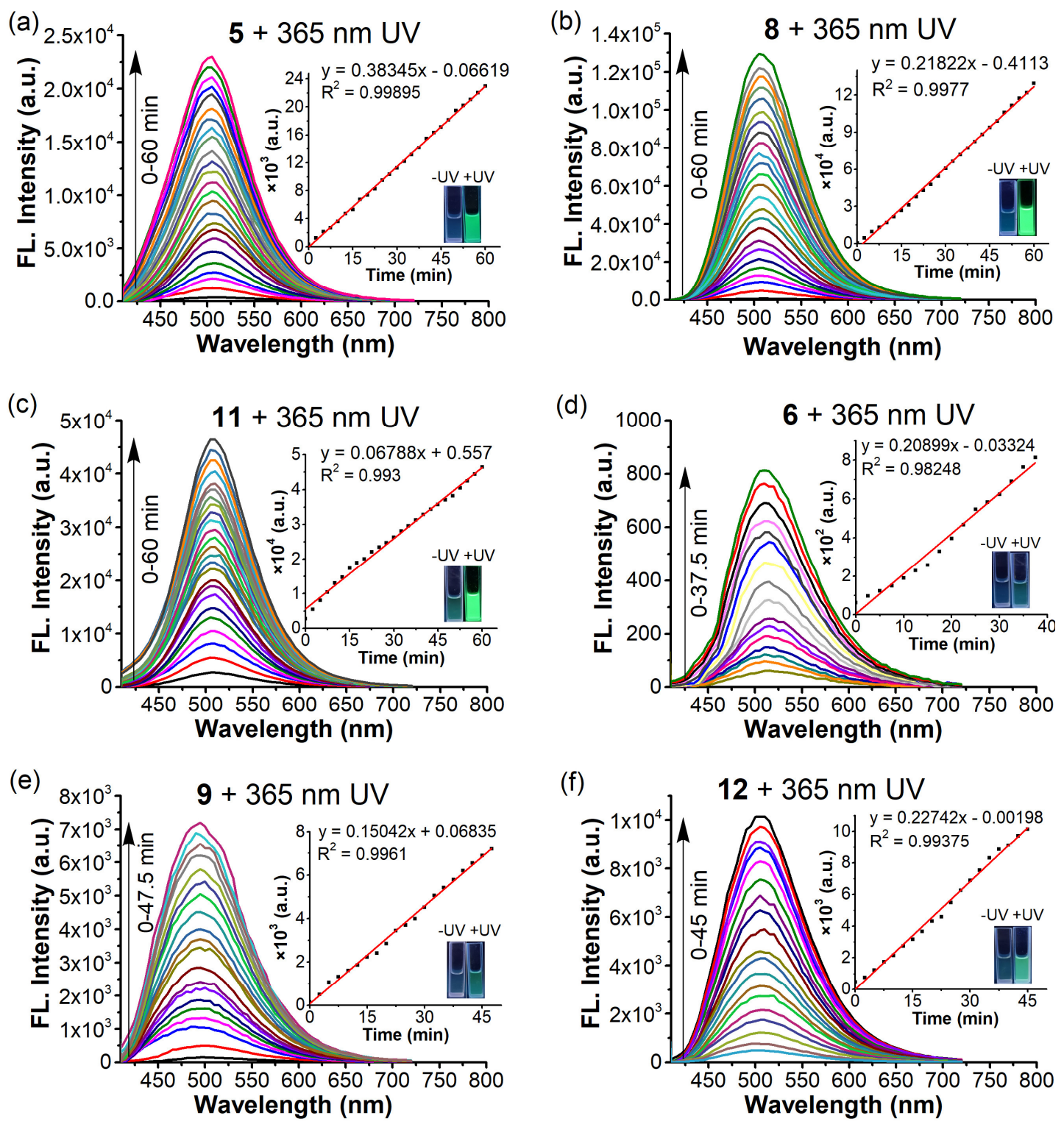

Figure S9. (a) Fluorescence emission spectra change of $10 \mu \mathrm{M} 5$ in $\mathrm{CH}_{3} \mathrm{CN}$ upon photoirradiation with $365 \mathrm{~nm} \mathrm{UV}$ for 0-60 $\min \left(\lambda_{\mathrm{exc}}=365 \mathrm{~nm}\right)$. (b) Fluorescence emission spectra change of $10 \mu \mathrm{M} 8$ in $\mathrm{CH}_{3} \mathrm{CN}$ upon photoirradiation with $365 \mathrm{~nm}$ UV for $0-60 \min \left(\lambda_{\mathrm{exc}}=365 \mathrm{~nm}\right)$. (c) Fluorescence emission spectra change of $10 \mu \mathrm{M}$ 11 in $\mathrm{CH}_{3} \mathrm{CN}$ upon photoirradiation with $365 \mathrm{~nm} \mathrm{UV}$ for $0-60 \min \left(\lambda_{\mathrm{exc}}=365 \mathrm{~nm}\right)$. (d) Fluorescence emission spectra change of $10 \mu \mathrm{M} 6$ in $\mathrm{CH}_{3} \mathrm{CN}$ upon photoirradiation with $365 \mathrm{~nm}$ UV for 0-37.5 $\min \left(\lambda_{\mathrm{exc}}=365 \mathrm{~nm}\right)$. (e) Fluorescence emission spectra change of $10 \mu \mathrm{M} 9$ in $\mathrm{CH}_{3} \mathrm{CN}$ upon photoirradiation with $365 \mathrm{~nm} \mathrm{UV}$ for 0-47.5 min $\left(\lambda_{\text {exc }}=365 \mathrm{~nm}\right)$. (f) Fluorescence emission spectra change of $10 \mu \mathrm{M} 12$ in $\mathrm{CH}_{3} \mathrm{CN}$ upon photoirradiation with $365 \mathrm{~nm}$ UV for $0-45 \min \left(\lambda_{\text {exc }}=365 \mathrm{~nm}\right)$. 
(a)
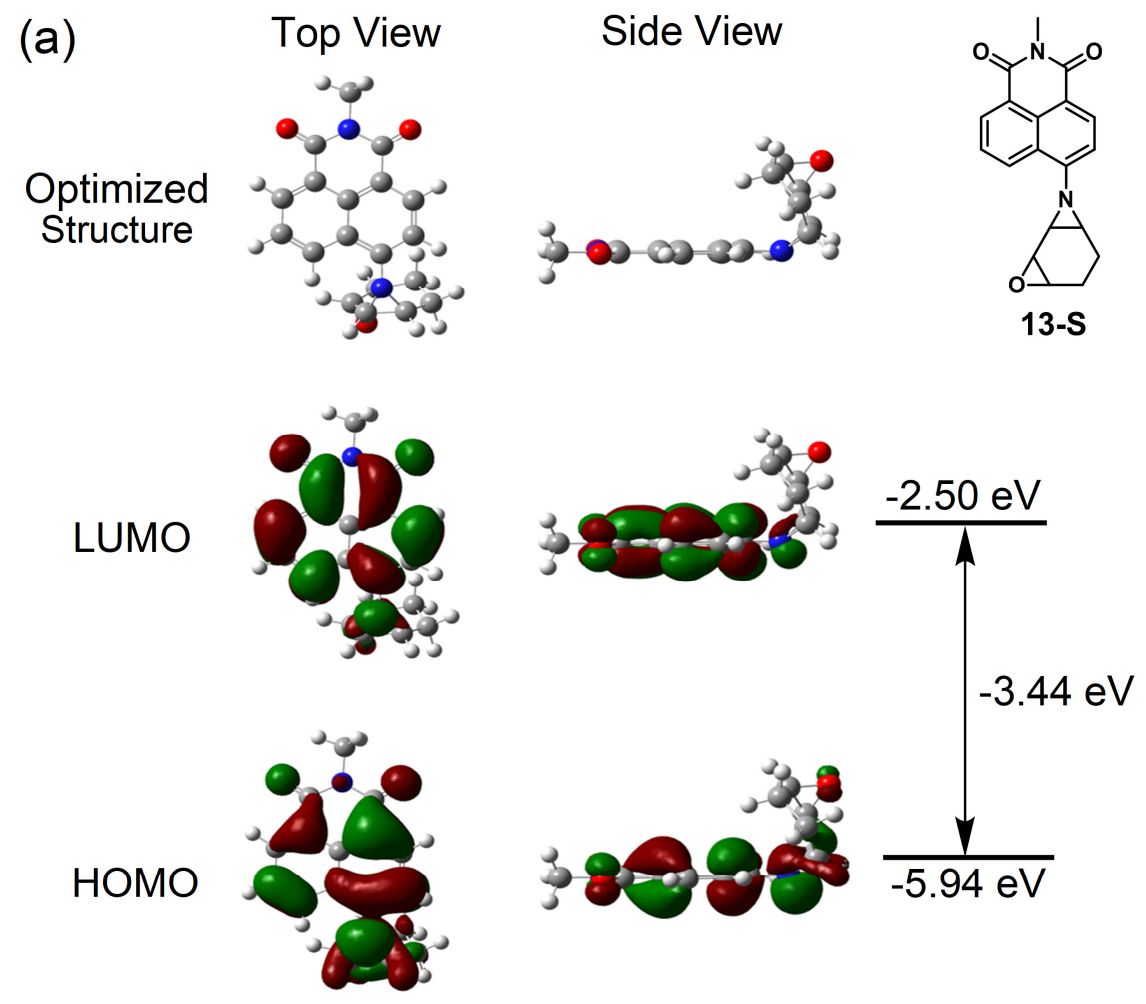

(b)

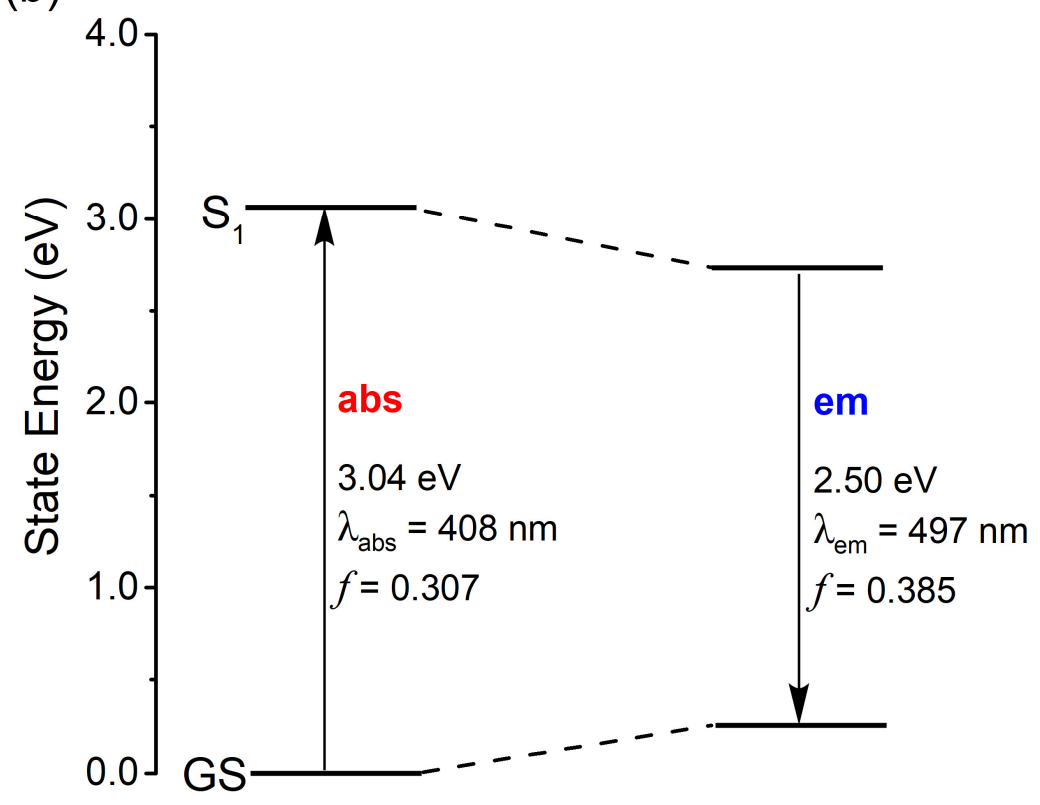

Figure S10. (a) Optimized geometry for 13-S, and contour plots of the HOMO and LUMO orbitals. (b) Energy diagram of 13-S with transitions oscillator strength values $(f)$. 


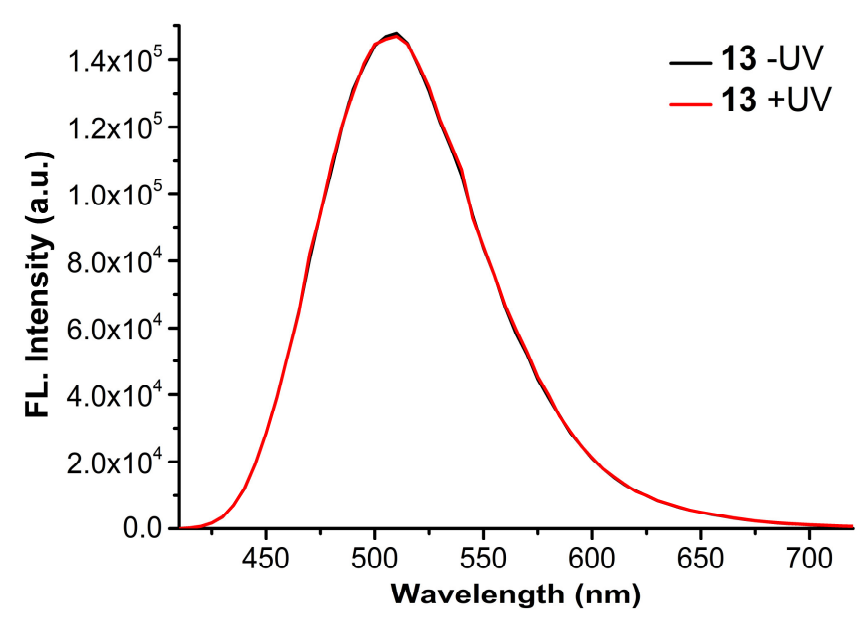

Figure S11. Photostability test of 13. Fluorescence emission spectra of $10 \mu \mathrm{M} 13$ in $\mathrm{CH}_{3} \mathrm{CN}$ without photoirradiation (black) or with photoirradiation using $365 \mathrm{~nm} \mathrm{UV}$ for $60 \mathrm{~min}(\mathrm{red})\left(\lambda_{\mathrm{exc}}=365 \mathrm{~nm}\right)$.

(a)

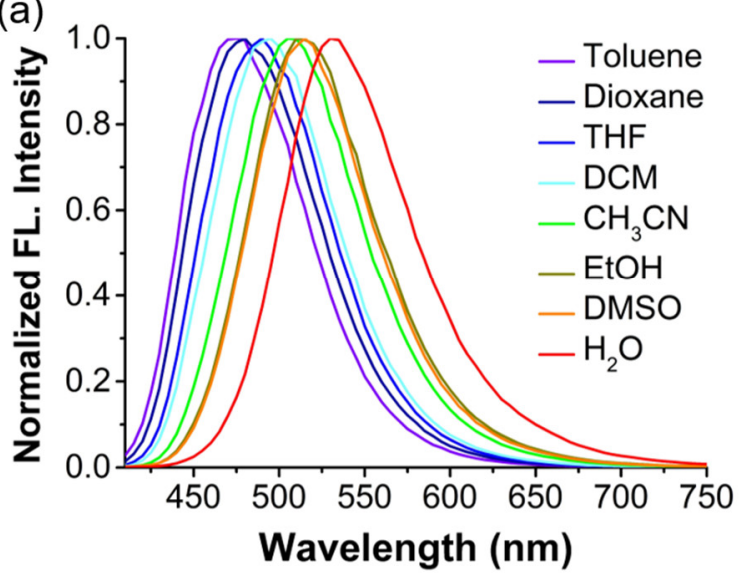

(b)

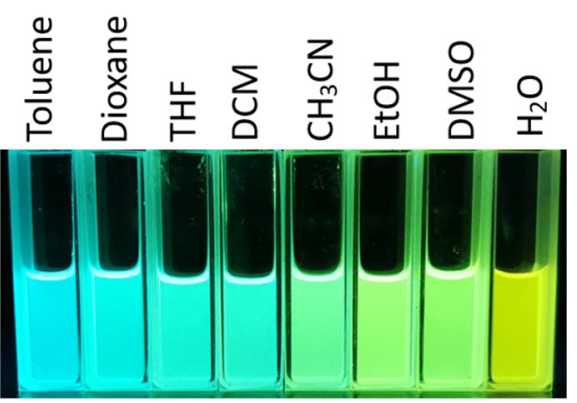

Figure S12. (a) Normalized Fluorescence emission spectra of 13 in different solvent (toluene/dioxane/THF/DCM/CH $\left.\mathrm{CH}_{3} \mathrm{CN} / \mathrm{EtOH} / \mathrm{DMSO} / \mathrm{H}_{2} \mathrm{O}\right)\left(\lambda_{\text {exc }}=365 \mathrm{~nm}\right.$ ). (b) Fluorescence images of $10 \mu \mathrm{M} 13$ solution in different solvent. The $365 \mathrm{~nm}$ light was irradiated from the top. 

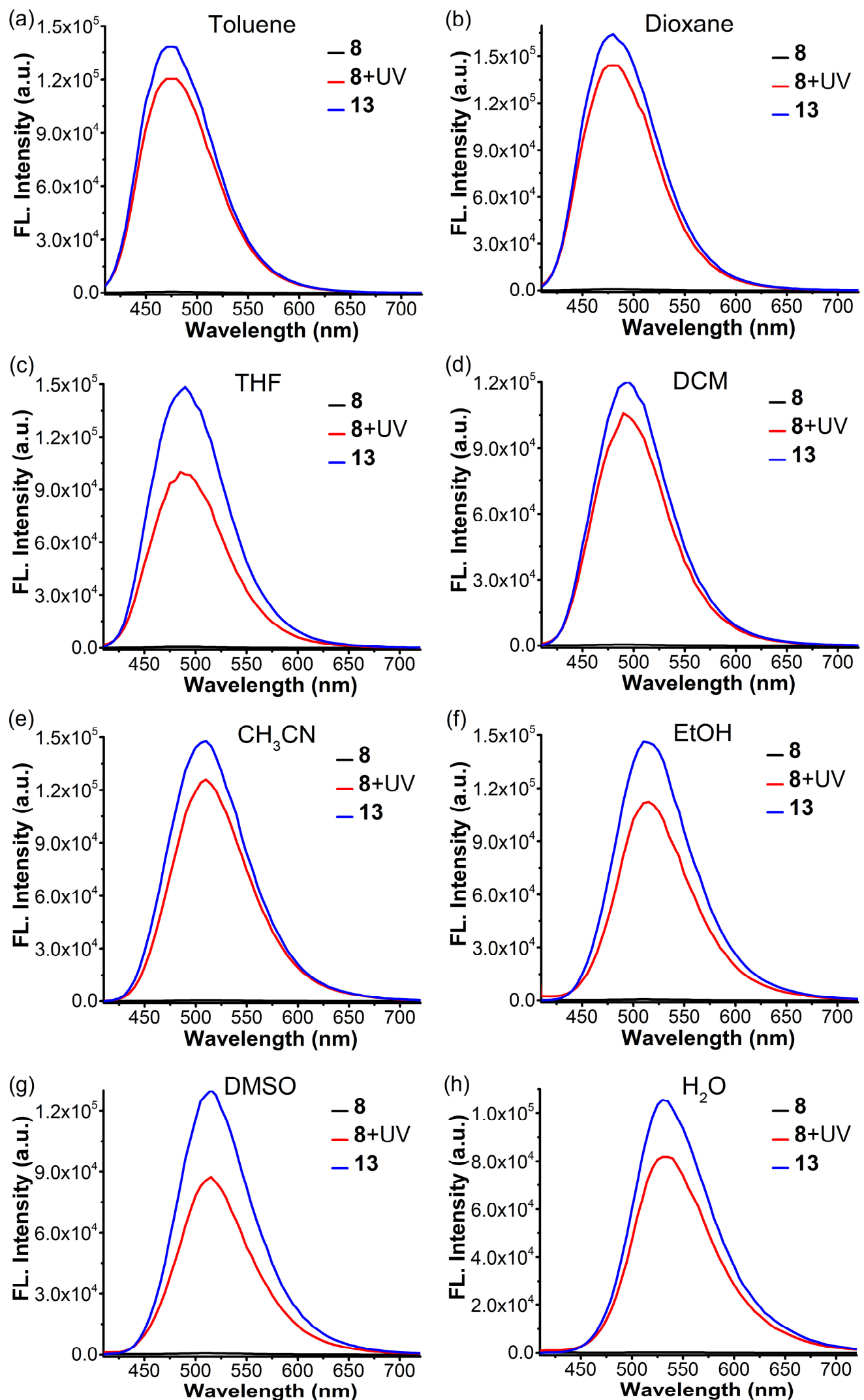

Figure S13. Fluorescence emission spectra of $10 \mu \mathrm{M} 8$ (black line), $10 \mu \mathrm{M} 13$ (blue line), and $10 \mu \mathrm{M} 8$ upon photoirradiation with $365 \mathrm{~nm} \mathrm{UV}$ for $60 \mathrm{~min}$ (red line) in different solvent (a) toluene; (b) dioxane; (c) THF; (d) DCM; (e) $\mathrm{CH}_{3} \mathrm{CN}$; (f) EtOH; (g) DMSO; (h) $\mathrm{H}_{2} \mathrm{O}\left(\lambda_{\text {exc }}=365 \mathrm{~nm}\right)$. 

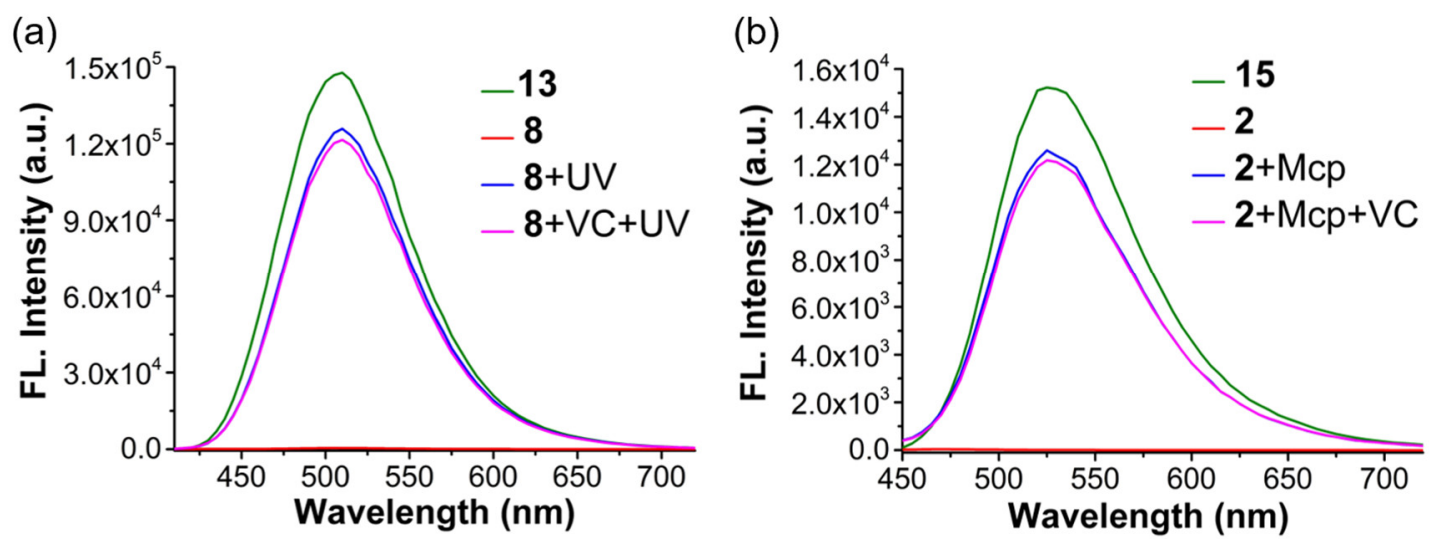

Figure S14. (a) Fluorescence emission spectra of $10 \mu \mathrm{M} 8$ (red line), $10 \mu \mathrm{M} 13$ (green line), $10 \mu \mathrm{M} 8$ upon photoirradiation with $365 \mathrm{~nm} \mathrm{UV}$ for $60 \mathrm{~min}$ in the presence (magenta line) or absence (blue line) of $100 \mu \mathrm{M}$ Vitamin $\mathrm{C}(\mathrm{VC})$ in $\mathrm{CH}_{3} \mathrm{CN}\left(\lambda_{\text {exc }}=\right.$ $365 \mathrm{~nm}$ ). (b) Fluorescence emission spectra of $10 \mu \mathrm{M} 2$ (red line), $10 \mu \mathrm{M} 15$ (green line), a mixture of $10 \mu \mathrm{M} 2$ and $50 \mu \mathrm{M}$ Mcp in the presence (magenta line) or absence (blue line) of $100 \mu \mathrm{M}$ Vitamin $\mathrm{C}(\mathrm{VC})$ in $\mathrm{CH}_{3} \mathrm{CN}\left(\lambda_{\mathrm{exc}}=365 \mathrm{~nm}\right)$.
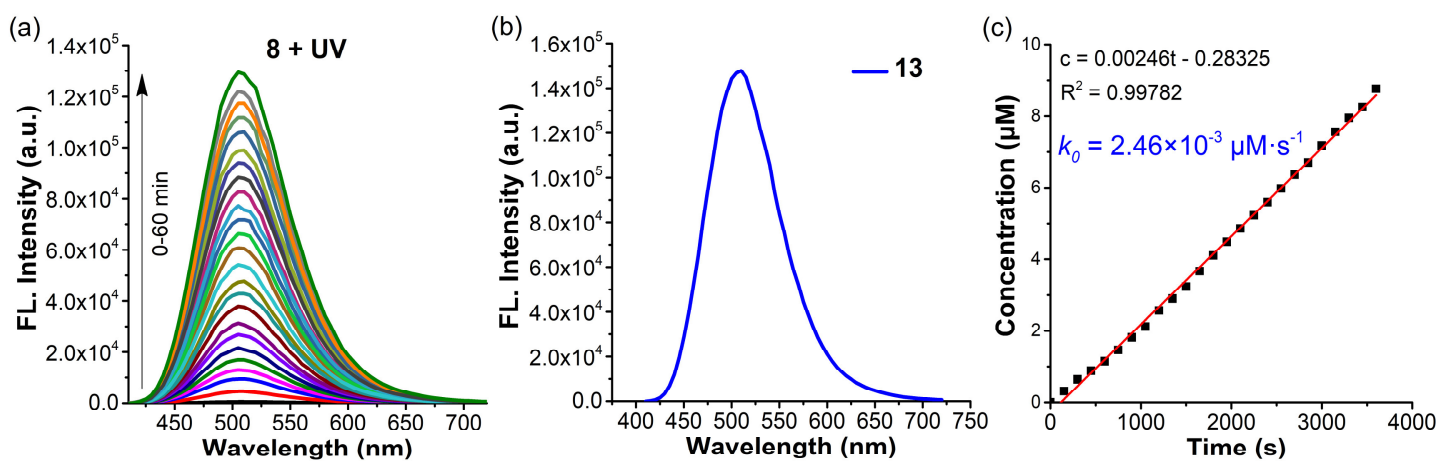

Figure S15. The rate of thermally induced rearrangement from 8 to 13. (a) Photo-induced fluorescence emission spectra change of $10 \mu \mathrm{M} 8$ in $\mathrm{CH}_{3} \mathrm{CN}$ upon photoirradiation with $365 \mathrm{~nm} \mathrm{UV}$ for 0-60 min (measured every $2.5 \mathrm{~min}$ ). (b) Emission spectra of $10 \mu \mathrm{M} 13$ in $\mathrm{CH}_{3} \mathrm{CN}\left(\lambda_{\mathrm{exc}}=365 \mathrm{~nm}\right)$. (c) Correlation of concentration of generated 13 from photoirradiating 8 with the photoirradiation time. The concentration of generated $\mathbf{1 3}$ at each time point $\left(C_{t}\right)$ was quantified using $10 \mu \mathrm{M}$ 13 as standard based on the equation below:

$$
C_{t}=C_{13} \times \frac{I_{t}}{I_{13}}
$$

where $I_{t}$ represents the fluorescence intensity of the photoirradiated solution of $10 \mu \mathrm{M}$ 8 at $505 \mathrm{~nm}\left(\lambda_{\text {exc }}=365 \mathrm{~nm}\right)$ at each time point; $I_{13}$ represents the fluorescence intensity of $10 \mu \mathrm{M} 13$ at $505 \mathrm{~nm}\left(\lambda_{\mathrm{exc}}=365 \mathrm{~nm}\right) ; C_{13}$ represents the concentration of 13, which is $10 \mu \mathrm{M}$. The zero-order reaction rate $\left(k_{0}\right)$ for the rearrangement reaction was obtained from the slope of the plot of the concentration of generated $\mathbf{1 3}$ versus photoirradiation time $\left(k_{0}=2.46 \times 10^{-3} \mu \mathrm{M} \cdot \mathrm{s}^{-1}\right)$ 

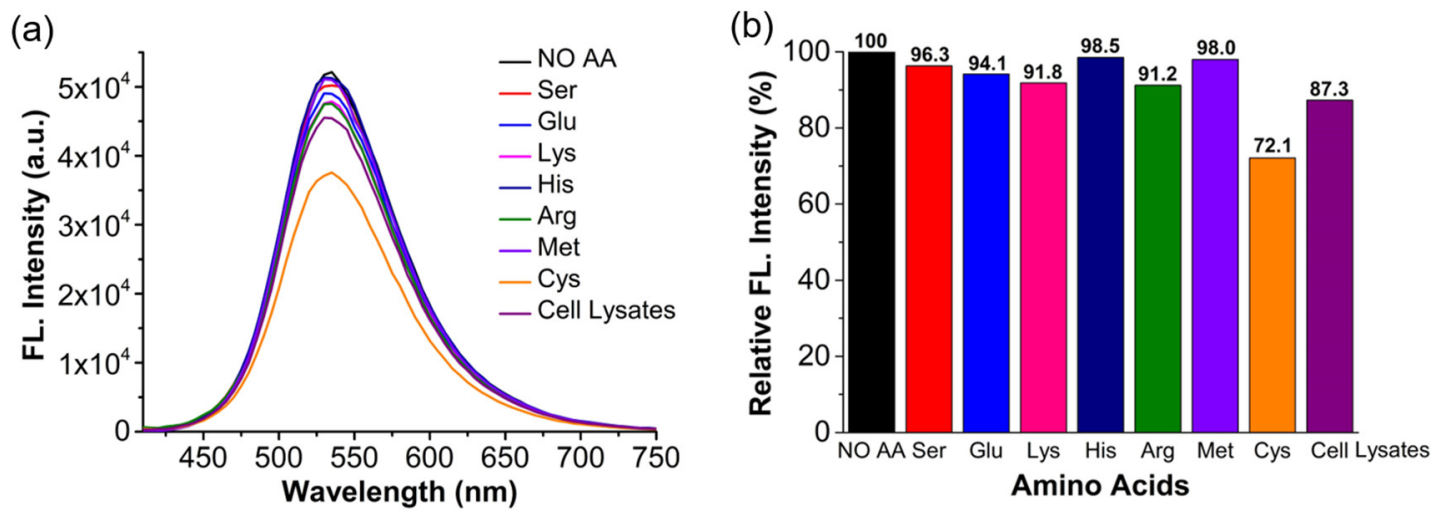

Figure S16. In situ fluorescence measurement for the nitroso-diene reaction in cell lysates or in the presence of nucleophilic amino acids. $10 \mu \mathrm{M} 2$ was mixed with 100 $\mu \mathrm{M} \quad$ Cyh $\quad$ and $\quad 100 \quad \mu \mathrm{M} \quad$ serine/glutamic acid/lysine/histidine/arginine/methionine/cysteine in PBS $(\mathrm{pH}=7.4)$ for $30 \mathrm{~min}$, or 10 $\mu \mathrm{M} 2$ and $100 \mu \mathrm{M} \mathbf{C y h}$ were incubated in HepG2 cell lysates $(2 \mathrm{mg} / \mathrm{mL}$ in PBS, $\mathrm{pH}=7.4$ ) for $30 \mathrm{~min}$, then the mixture was irradiated by $365 \mathrm{~nm} \mathrm{UV}$ for $30 \mathrm{~min}$. (a) Fluorescence emission spectra of the nitroso-diene reaction in cell lysates or in the presence of nucleophilic amino acids $\left(\lambda_{\text {exc }}=365 \mathrm{~nm}\right)$. (b) Relative fluorescence intensity of the nitroso-diene reaction in cell lysates or in the presence of nucleophilic amino acids at the $\lambda_{\max }(535 \mathrm{~nm})\left(\lambda_{\mathrm{exc}}=365 \mathrm{~nm}\right)$.

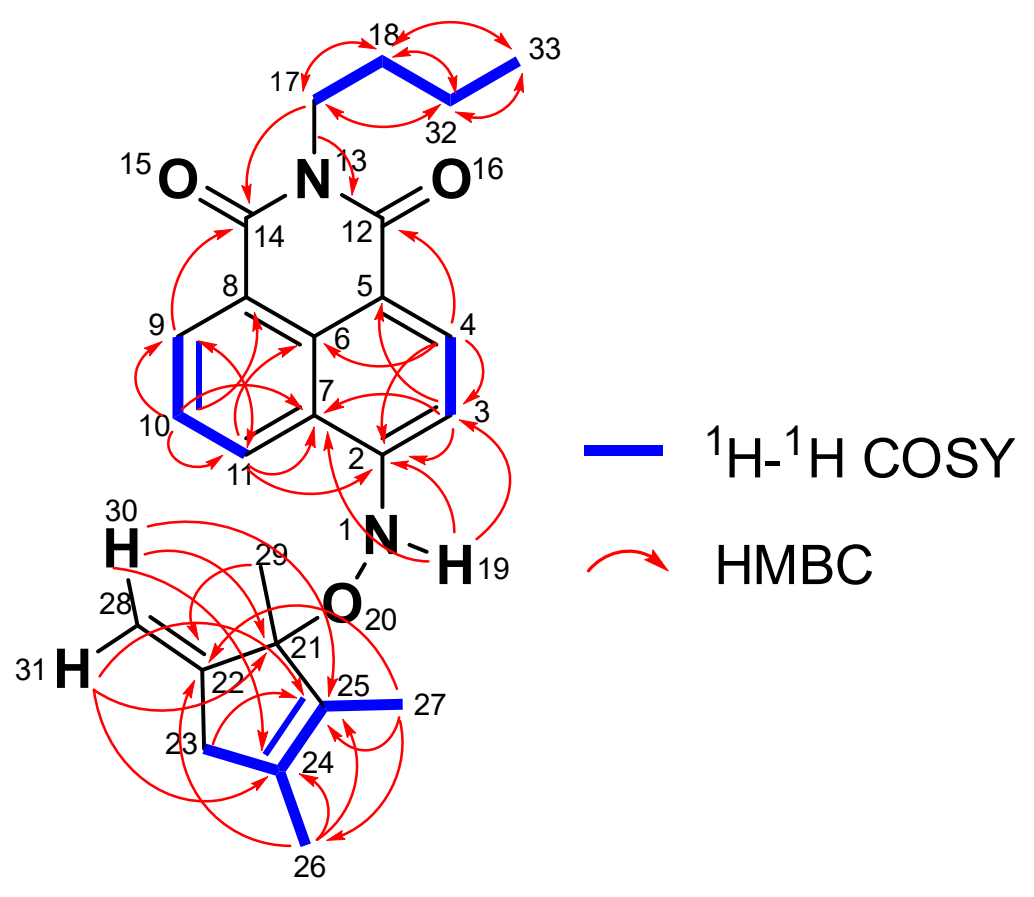

Figure S17. Key ${ }^{1} \mathrm{H}-{ }^{1} \mathrm{H}$ COSY and HMBC correlations of $\mathbf{1 5 .}$ 

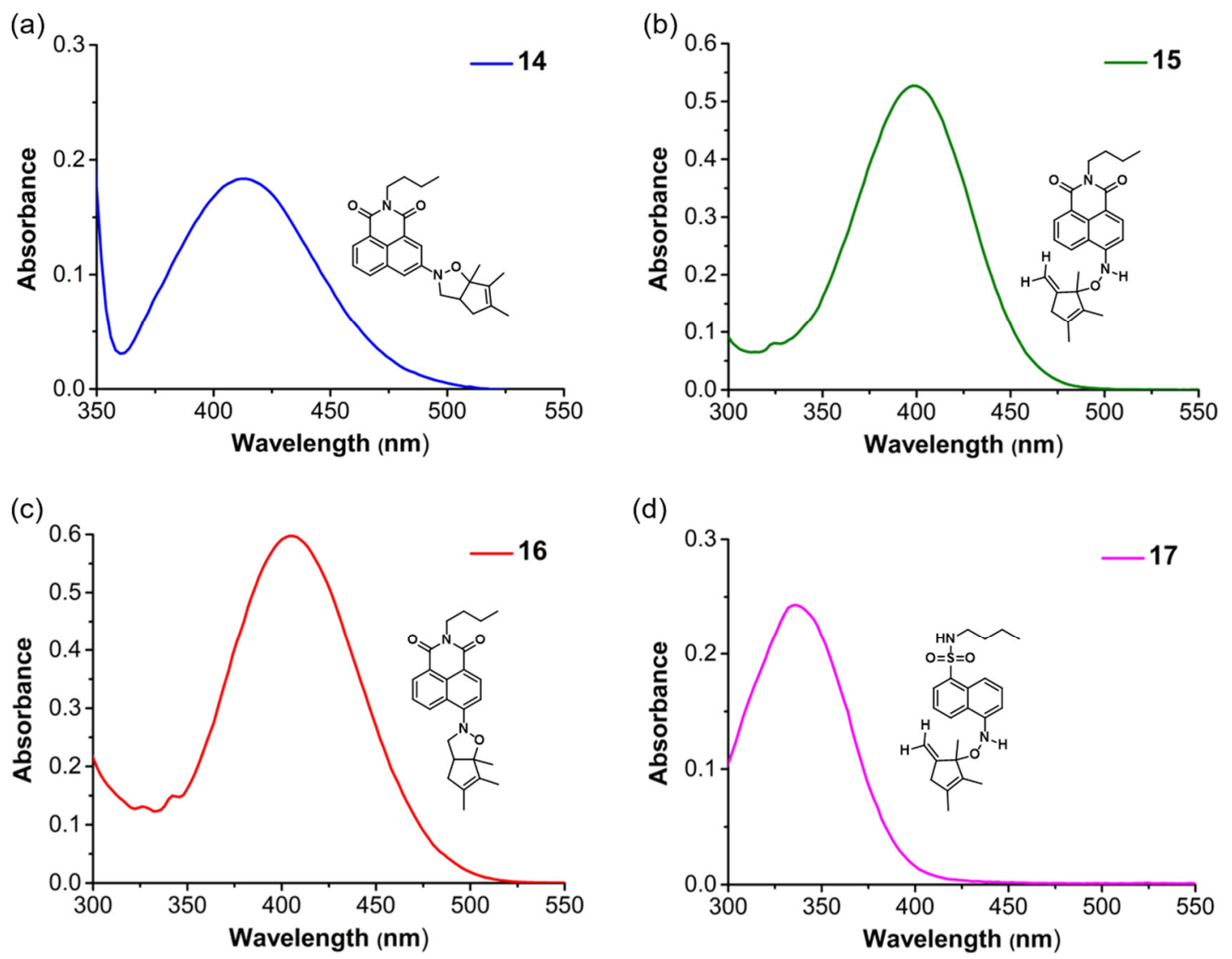

Figure S18. Absorption spectra of 14 (a), 15 (b), 16 (c), and 17 (d).
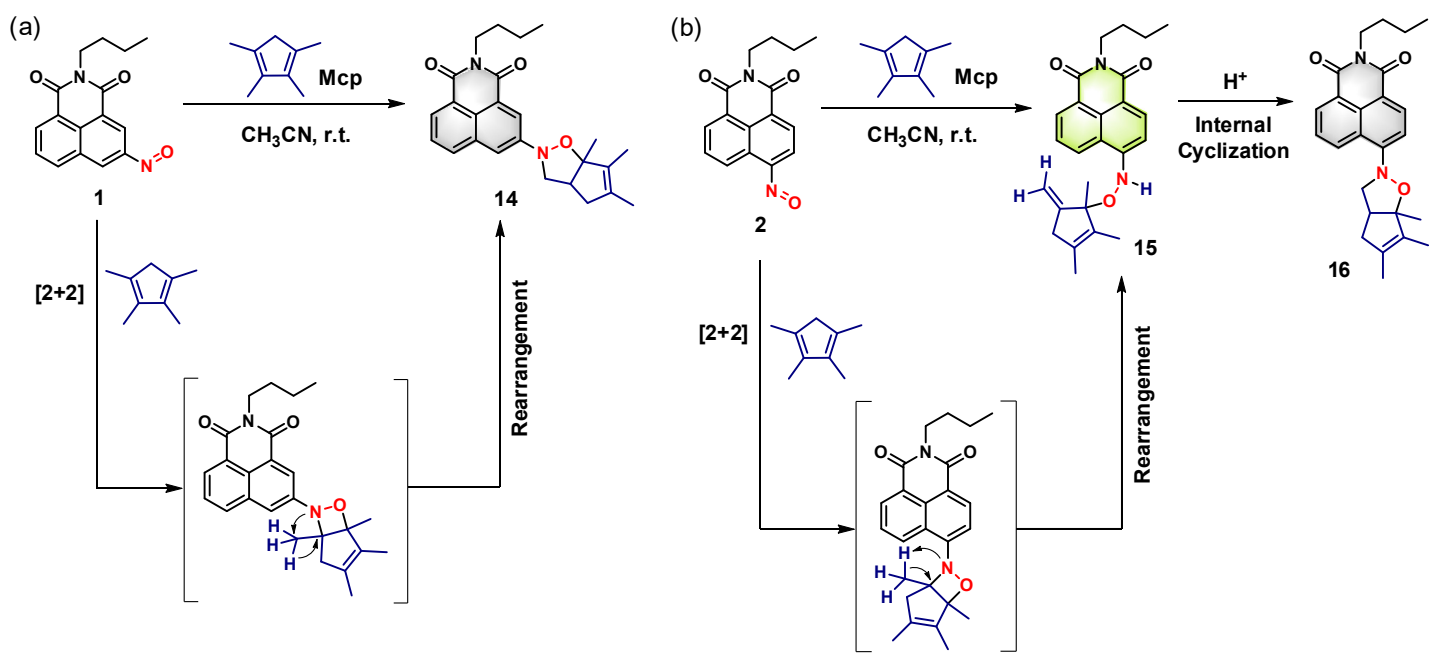

Figure S19. (a) Proposed reaction pathway for the formation of 14. (b) Proposed reaction pathway for the formation of $\mathbf{1 5}$. 

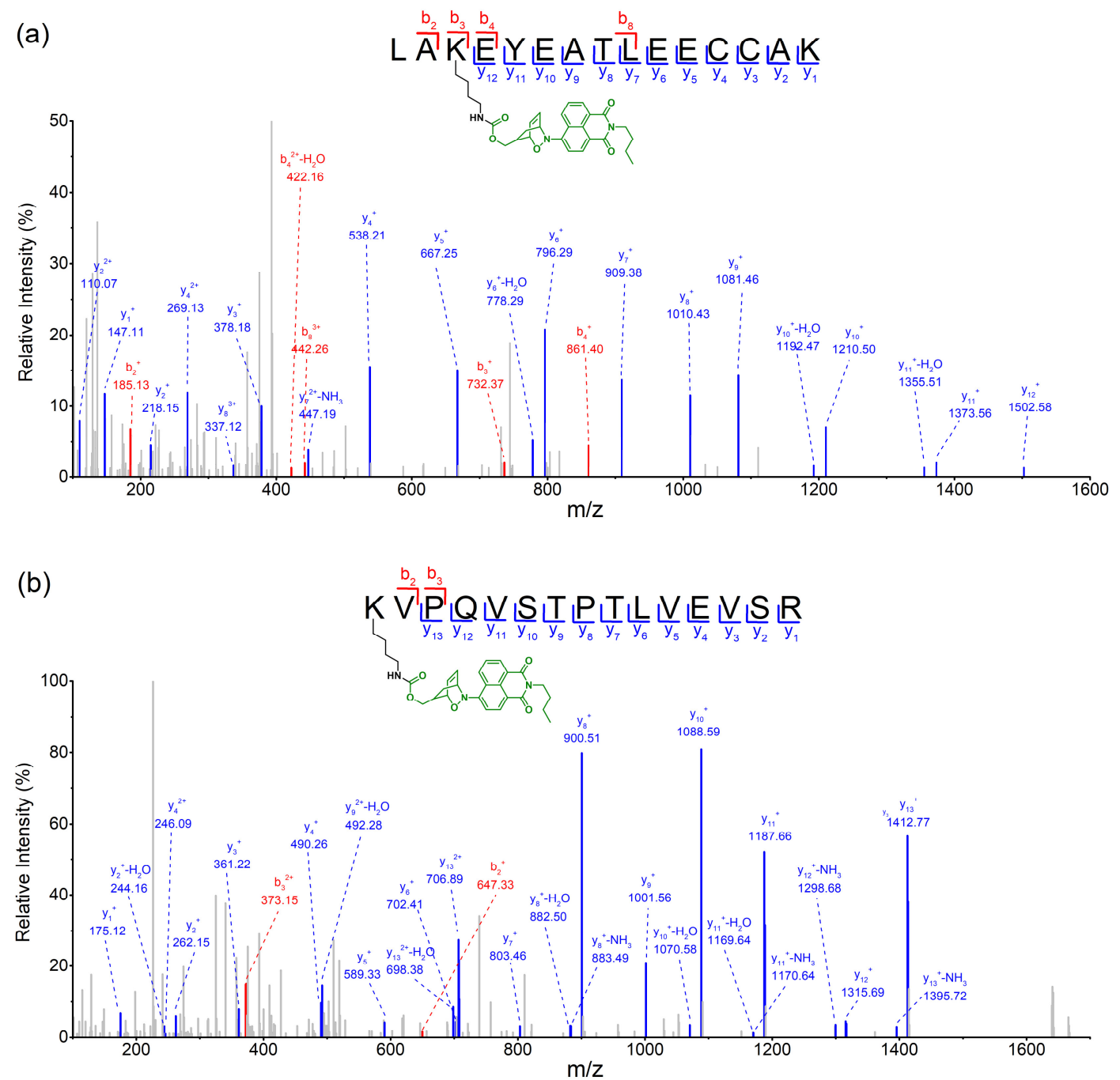

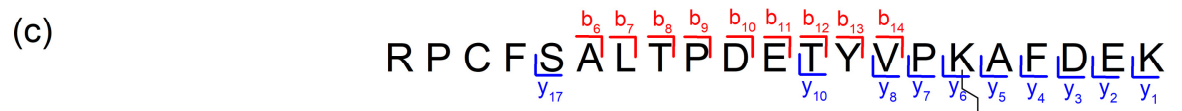

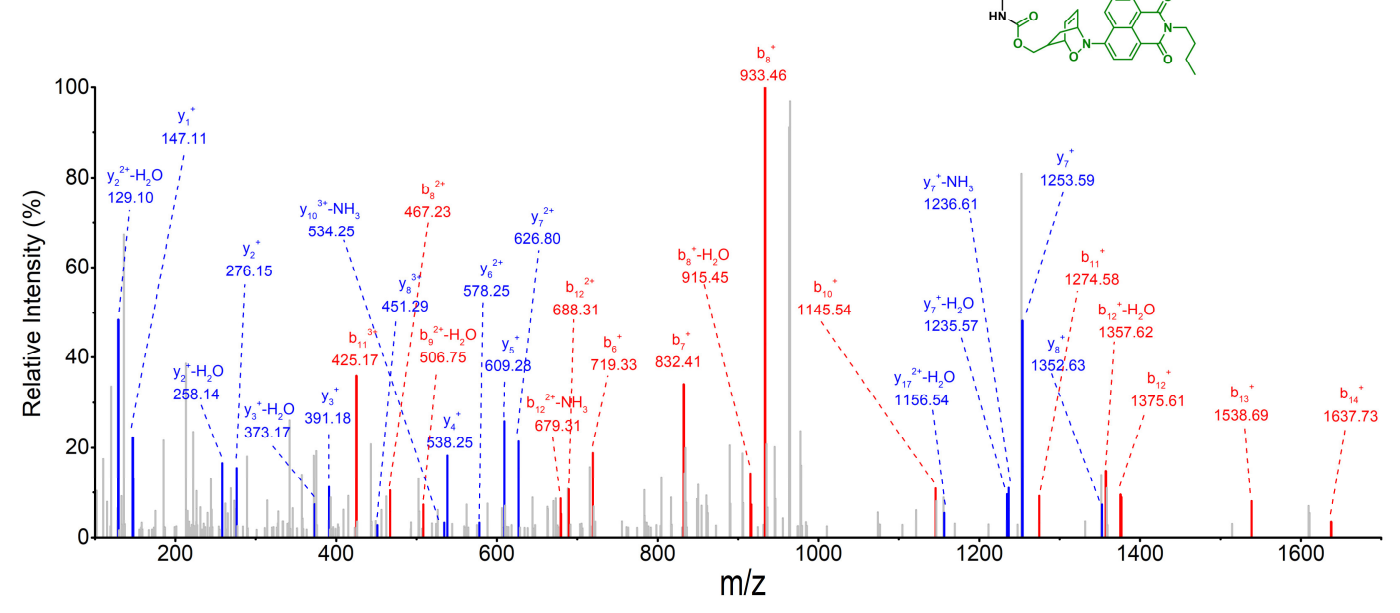


(d)

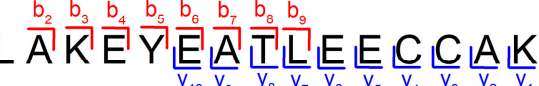

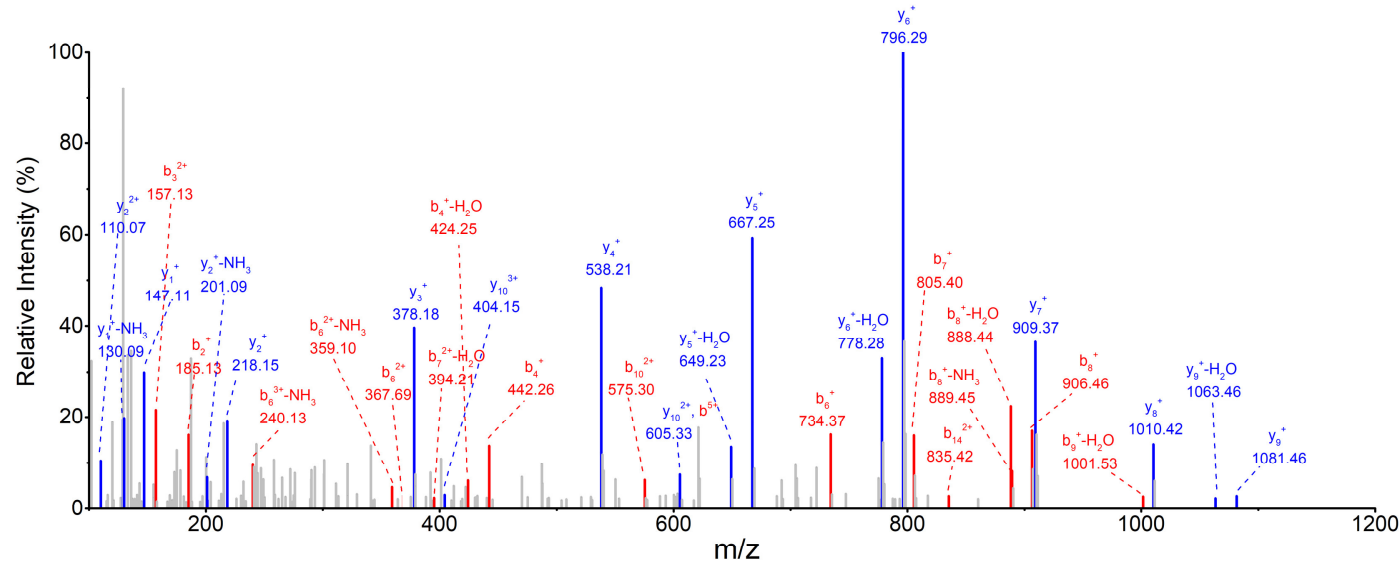

(e)

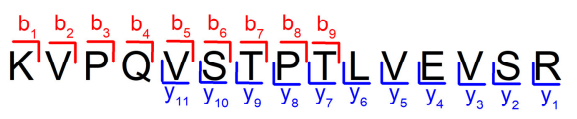

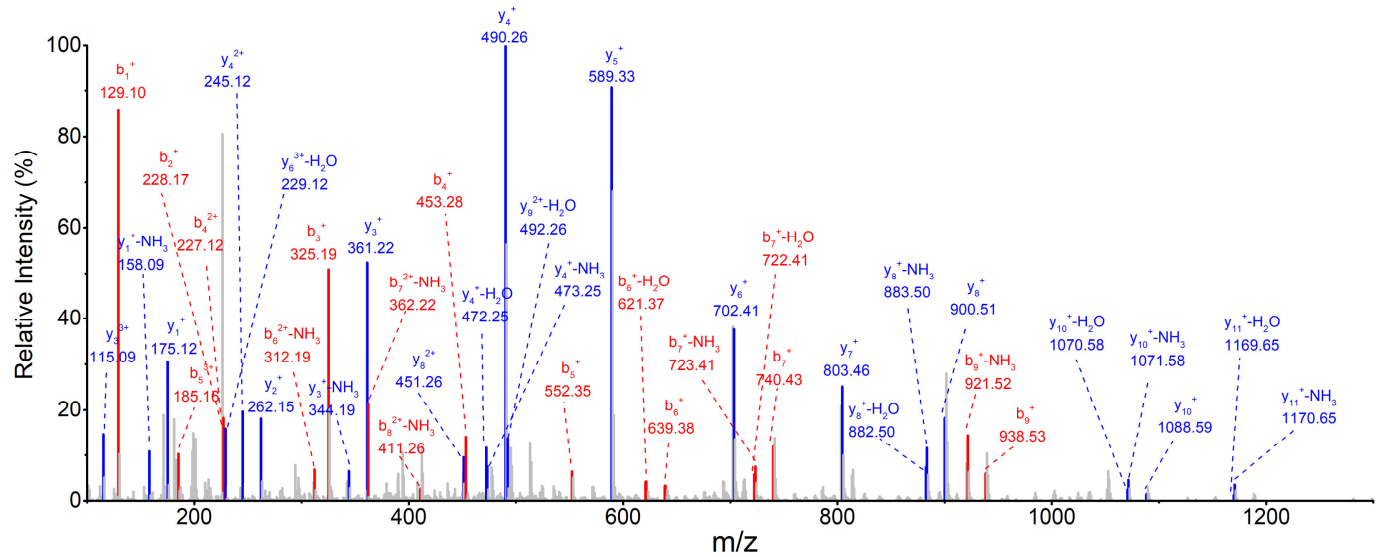

(f)

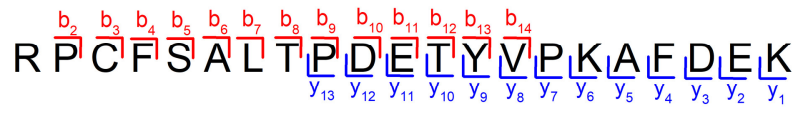

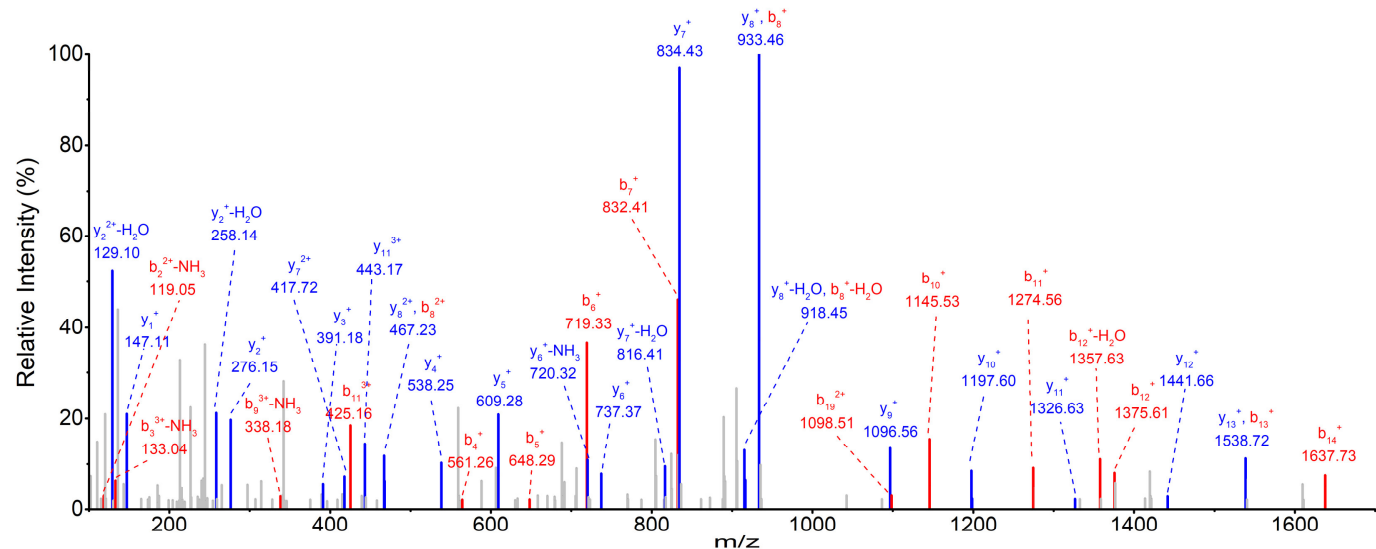

Figure S20. MS/MS spectrum for the peptides from BSA-Cyh labeled with 2 (a-c) and the same peptides from unmodified BSA labeled with $\mathbf{2}$ (d-f). 
Table S1. Photophysical properties of naphthylene probes. ${ }^{\mathrm{a}}$

\begin{tabular}{|c|c|c|c|c|c|c|}
\hline Probes & $\lambda_{\mathrm{abs}}$ & $\lambda_{\mathrm{em}}^{\mathrm{b}}$ & Stokes shift & $\varepsilon\left[\mathrm{M}^{-1} \mathrm{~cm}^{-1}\right]$ & $\Phi_{\mathrm{F}}^{\mathrm{c}}$ & Turn-on $^{\mathrm{d}}$ \\
\hline 1 & 396 & 470 & 74 & 10023 & 0.002 & --- \\
\hline 2 & 380 & 470 & 90 & 10352 & 0.002 & --- \\
\hline 3 & 366 & 479 & 113 & 13137 & 0.001 & --- \\
\hline 4 & 404 & 496 & 92 & 9207 & 0.003 & 2.8 \\
\hline 5 & 384 & 510 & 126 & 10733 & 0.009 & 8.9 \\
\hline $\mathbf{5}-\mathbf{U} \mathbf{V}^{\mathrm{e}}$ & 386 & 505 & 119 & 10837 & 0.14 & 561 \\
\hline 6 & 322 & 513 & 191 & 9040 & 0.004 & 4.4 \\
\hline 6-UV $\mathbf{V}^{\mathrm{e}}$ & 322 & 510 & 188 & 7783 & 0.02 & 58 \\
\hline 7 & 404 & 506 & 102 & 10007 & 0.02 & 10.9 \\
\hline 8 & 406 & 510 & 104 & 10907 & 0.02 & 11.8 \\
\hline 8-UV $\mathbf{V}^{\mathrm{e}}$ & 386 & 505 & 119 & 10097 & 0.85 & 3252 \\
\hline 9 & 346 & 501 & 155 & 3880 & 0.003 & 10.4 \\
\hline 9-UV $\mathbf{V}^{\mathrm{e}}$ & 336 & 497 & 161 & 4097 & 0.08 & 514 \\
\hline 10 & 404 & 502 & 98 & 9337 & 0.01 & 5.5 \\
\hline 11 & 402 & 510 & 108 & 12043 & 0.05 & 66 \\
\hline 11-UV & 378 & 505 & 127 & 12203 & 0.28 & 1132 \\
\hline 12 & 336 & 504 & 168 & 5117 & 0.02 & 35 \\
\hline $12-U V^{\mathrm{e}}$ & 324 & 507 & 183 & 6273 & 0.1 & 725 \\
\hline 13 & 386 & 505 & 119 & 11890 & 0.92 & 3604 \\
\hline 14 & 413 & 550 & 137 & 4427 & 0.008 & 2.9 \\
\hline 15 & 398 & 526 & 128 & 9673 & 0.12 & 371 \\
\hline 16 & 404 & 541 & 137 & 11187 & 0.009 & --- \\
\hline 17 & 336 & 518 & 182 & 6373 & 0.1 & 713 \\
\hline
\end{tabular}

${ }^{\mathrm{a}} \mathrm{All}$ test samples were prepared at the concentration of $10 \mu \mathrm{M}$ in $\mathrm{CH}_{3} \mathrm{CN}$. ${ }^{\mathrm{b}}$ Determined at $\lambda_{\mathrm{exc}}=365 \mathrm{~nm}$. cFluorescence quantum yield, quinine sulfate in aqueous $0.1 \mathrm{~N} \mathrm{H}_{2} \mathrm{SO}_{4}$ as standard. ${ }^{\mathrm{d}}$ Fluorescence turn-on ratios of

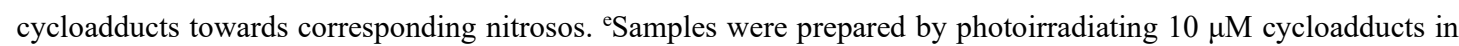
$\mathrm{CH}_{3} \mathrm{CN}$ with $365 \mathrm{~nm} \mathrm{UV}$ light for $60 \mathrm{~min}$. 
Table S2. Crystal data and structure refinement for $\mathbf{8}$.

\begin{tabular}{|c|c|}
\hline & Single crystal of 8 \\
\hline Empirical formula & $\mathrm{C}_{22} \mathrm{H}_{22} \mathrm{~N}_{2} \mathrm{O}_{3}$ \\
\hline Formula weight & 362.41 \\
\hline Temperature/K & $100.00(10)$ \\
\hline Crystal system & monoclinic \\
\hline Space group & $\mathrm{P} 2{ }_{1} / \mathrm{c}$ \\
\hline $\mathrm{a} / \AA \AA$ & $15.0099(6)$ \\
\hline $\mathrm{b} / \AA ̊$ & $17.0809(7)$ \\
\hline $\mathrm{c} / \AA \AA$ & $7.1123(2)$ \\
\hline$\alpha /^{\circ}$ & 90 \\
\hline$\beta /^{\circ}$ & $101.994(4)$ \\
\hline$\gamma /{ }^{\circ}$ & 90 \\
\hline Volume $/ \AA^{3}$ & $1783.67(12)$ \\
\hline $\mathrm{Z}$ & 4 \\
\hline$\rho_{\text {calc }} \mathrm{g} / \mathrm{cm}^{3}$ & 1.350 \\
\hline$\mu / \mathrm{mm}^{-1}$ & 0.729 \\
\hline $\mathrm{F}(000)$ & 768.0 \\
\hline Crystal size $/ \mathrm{mm}^{3}$ & $0.13 \times 0.12 \times 0.11$ \\
\hline Radiation & $\mathrm{Cu} \mathrm{K \alpha}(\lambda=1.54184)$ \\
\hline $2 \Theta$ range for data collection $/^{\circ}$ & 6.02 to 147.944 \\
\hline Index ranges & $-18 \leq \mathrm{h} \leq 18,-19 \leq \mathrm{k} \leq 21,-8 \leq 1 \leq 6$ \\
\hline Reflections collected & 6835 \\
\hline Independent reflections & $3506\left[\mathrm{R}_{\text {int }}=0.0288, \mathrm{R}_{\text {sigma }}=0.0380\right]$ \\
\hline Data/restraints/parameters & $3506 / 0 / 245$ \\
\hline Goodness-of-fit on $\mathrm{F}^{2}$ & 1.034 \\
\hline Final $R$ indexes $[\mathrm{I}>=2 \sigma(\mathrm{I})]$ & $\mathrm{R}_{1}=0.0485, \mathrm{wR}_{2}=0.1264$ \\
\hline Final $\mathrm{R}$ indexes [all data] & $\mathrm{R}_{1}=0.0567, \mathrm{wR}_{2}=0.1345$ \\
\hline Largest diff. peak/hole / e $\AA^{-3}$ & $0.22 /-0.30$ \\
\hline
\end{tabular}


Table S3. Crystal data and structure refinement for 13.

\begin{tabular}{|c|c|}
\hline & Single crystal of $\mathbf{1 3}$ \\
\hline Empirical formula & $\mathrm{C}_{22} \mathrm{H}_{22} \mathrm{~N}_{2} \mathrm{O}_{3}$ \\
\hline Formula weight & 362.41 \\
\hline Temperature/K & $100.01(10)$ \\
\hline Crystal system & orthorhombic \\
\hline Space group & Pbca \\
\hline $\mathrm{a} / \AA \AA$ & $8.3233(16)$ \\
\hline $\mathrm{b} / \AA$ & $13.736(3)$ \\
\hline $\mathrm{c} / \AA \AA$ & $30.944(5)$ \\
\hline$\alpha /^{\circ}$ & 90 \\
\hline$\beta /^{\circ}$ & 90 \\
\hline$\gamma /{ }^{\circ}$ & 90 \\
\hline Volume $/ \AA^{3}$ & $3537.8(11)$ \\
\hline $\mathrm{Z}$ & 8 \\
\hline$\rho_{\text {calc }} \mathrm{g} / \mathrm{cm}^{3}$ & 1.361 \\
\hline$\mu / \mathrm{mm}^{-1}$ & 0.735 \\
\hline $\mathrm{F}(000)$ & 1536.0 \\
\hline Crystal size $/ \mathrm{mm}^{3}$ & $0.15 \times 0.1 \times 0.08$ \\
\hline Radiation & $\mathrm{Cu} \mathrm{K \alpha}(\lambda=1.54184)$ \\
\hline $2 \Theta$ range for data collection ${ }^{\circ}$ & 5.712 to 147.052 \\
\hline Index ranges & $-9 \leq \mathrm{h} \leq 10,-16 \leq \mathrm{k} \leq 16,-37 \leq 1 \leq 35$ \\
\hline Reflections collected & 8774 \\
\hline Independent reflections & $3474\left[\mathrm{R}_{\text {int }}=0.1061, \mathrm{R}_{\text {sigma }}=0.1265\right]$ \\
\hline Data/restraints/parameters & $3474 / 64 / 239$ \\
\hline Goodness-of-fit on $\mathrm{F}^{2}$ & 1.032 \\
\hline Final $R$ indexes $[\mathrm{I}>=2 \sigma(\mathrm{I})]$ & $\mathrm{R}_{1}=0.1627, \mathrm{wR}_{2}=0.3285$ \\
\hline Final $\mathrm{R}$ indexes [all data] & $\mathrm{R}_{1}=0.1771, \mathrm{wR}_{2}=0.3444$ \\
\hline Largest diff. peak/hole / e $\AA^{-3}$ & $1.22 /-0.61$ \\
\hline
\end{tabular}


Table S4. Crystal data and structure refinement for $\mathbf{1 4}$.

\begin{tabular}{|c|c|}
\hline & Single crystal of $\mathbf{1 4}$ \\
\hline Empirical formula & $\mathrm{C}_{25} \mathrm{H}_{28} \mathrm{~N}_{2} \mathrm{O}_{3}$ \\
\hline Formula weight & 404.49 \\
\hline Temperature/K & $199.98(19)$ \\
\hline Crystal system & monoclinic \\
\hline Space group & $\mathrm{I} 2 / \mathrm{a}$ \\
\hline $\mathrm{a} / \AA$ & $16.1760(11)$ \\
\hline $\mathrm{b} / \AA ̊$ & $8.1828(4)$ \\
\hline $\mathrm{c} / \AA ̊$ & $32.3558(16)$ \\
\hline$\alpha /^{\circ}$ & 90 \\
\hline$\beta /{ }^{\circ}$ & $92.290(5)$ \\
\hline$\gamma /{ }^{\circ}$ & 90 \\
\hline Volume $/ \AA^{3}$ & $4279.3(4)$ \\
\hline $\mathrm{Z}$ & 8 \\
\hline$\rho_{\text {calc }} \mathrm{g} / \mathrm{cm}^{3}$ & 1.256 \\
\hline$\mu / \mathrm{mm}^{-1}$ & 0.083 \\
\hline $\mathrm{F}(000)$ & 1728.0 \\
\hline Crystal size $/ \mathrm{mm}^{3}$ & $0.14 \times 0.13 \times 0.12$ \\
\hline Radiation & Mo $K \alpha(\lambda=0.71073)$ \\
\hline $2 \Theta$ range for data collection ${ }^{\circ}$ & 5.04 to 49.99 \\
\hline Index ranges & $-19 \leq \mathrm{h} \leq 17,-9 \leq \mathrm{k} \leq 8,-38 \leq 1 \leq 32$ \\
\hline Reflections collected & 9222 \\
\hline Independent reflections & $3780\left[\mathrm{R}_{\text {int }}=0.0502, \mathrm{R}_{\text {sigma }}=0.0694\right]$ \\
\hline Data/restraints/parameters & $3780 / 0 / 275$ \\
\hline Goodness-of-fit on $\mathrm{F}^{2}$ & 1.025 \\
\hline Final $\mathrm{R}$ indexes $[\mathrm{I}>=2 \sigma(\mathrm{I})]$ & $\mathrm{R}_{1}=0.0576, \mathrm{wR}_{2}=0.1307$ \\
\hline Final $\mathrm{R}$ indexes [all data] & $\mathrm{R}_{1}=0.0875, \mathrm{wR}_{2}=0.1521$ \\
\hline Largest diff. peak/hole / e $\AA^{-3}$ & $0.19 /-0.21$ \\
\hline
\end{tabular}




\section{General Information}

Solvents and chemicals were purchased from commercial sources and used directly without furtherpurification. ${ }^{1} \mathrm{H}$ NMR spectra were recorded on a Varian $400 \mathrm{MHz}$ NMR spectrometer. ${ }^{13} \mathrm{CNMR}$ spectra were recorded on a Varian500 $\mathrm{MHz} \mathrm{NMR}$ spectrometer. Chemical shifts are referenced to the residual solvent peak and reported as $\delta$ units in ppm (in NMR description, $\mathrm{s}=$ singlet, $\mathrm{d}=$ doublet, $\mathrm{t}=$ triplet, $\mathrm{q}=$ quartet and $\mathrm{m}=$ multiple), and all coupling constant $(J)$ values are given in hertz. ESI-HRMS data were measured on Thermo LCQ Deca XP Max mass spectrometer equipped with an ion trap mass analyzer. Silica gel flash column chromatography was performed on Biotage Isolera one. Fluorescence emission spectra and full wavelength absorption spectra were recorded on Tecan Spark ${ }^{\mathrm{TM}}$ 10M Multimode Microplate Reader. Confocal laser scanning microscope imaging was conducted using a Leica TCS SP8 X Confocal Microscope.

\section{Experimental Procedures and Characterization Data}

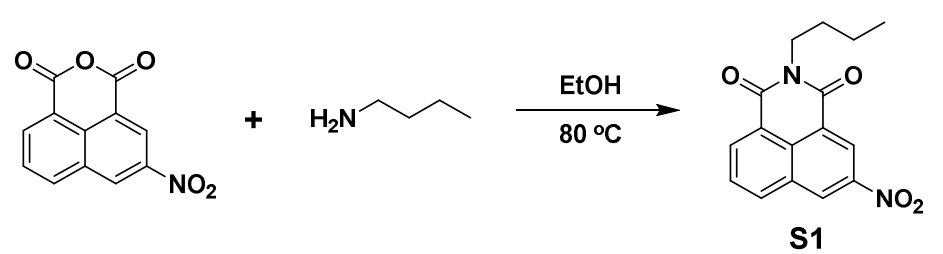

\section{Synthesis of 2-butyl-5-nitro-1H-benzo[de]isoquinoline-1,3(2H)-dione (S1)}

To a solution of 3- $\mathrm{NO}_{2}-1,8$-naphthalic anhydride $(2 \mathrm{~g}, 8.22 \mathrm{mmol})$ in EtOH $(30 \mathrm{~mL})$ was added $n$-butylamine ( $600 \mathrm{mg}, 8.22 \mathrm{mmol}$ ). The reaction mixture was stirred at 80 ${ }^{\circ} \mathrm{C}$ using an oil bath for $4 \mathrm{~h}$, then concentrated. The residue was purified by silica gel flash column chromatography $(\mathrm{PE} / \mathrm{EtOAc}=2: 1)$ to afford the titled compound as white solid (1.1 g, 45\% yield). m.p.: $188-190{ }^{\circ} \mathrm{C} ;{ }^{1} \mathrm{H}$ NMR $\left(400 \mathrm{MHz}, \mathrm{CDCl}_{3}\right) \delta 9.31$ $(\mathrm{d}, J=2.2 \mathrm{~Hz}, 1 \mathrm{H}), 9.13(\mathrm{~d}, J=2.3 \mathrm{~Hz}, 1 \mathrm{H}), 8.78(\mathrm{~d}, J=7.3 \mathrm{~Hz}, 1 \mathrm{H}), 8.42(\mathrm{~d}, J=9.2$ $\mathrm{Hz}, 1 \mathrm{H}), 7.99-7.90(\mathrm{~m}, 1 \mathrm{H}), 4.25-4.16(\mathrm{~m}, 2 \mathrm{H}), 1.79-1.69(\mathrm{~m}, 2 \mathrm{H}), 1.52-1.42$ $(\mathrm{m}, 2 \mathrm{H}), 0.99(\mathrm{t}, J=7.3 \mathrm{~Hz}, 3 \mathrm{H}) ;{ }^{13} \mathrm{C} \mathrm{NMR}\left(101 \mathrm{MHz}, \mathrm{CDCl}_{3}\right) \delta 163.2,162.6,146.5$, 135.6, 134.5, 131.1, 130.3, 129.2, 128.9, 124.9, 124.3, 123.4, 40.7, 30.2, 20.4, 13.9; HRMS (ESI) m/z: [M+ H] $]^{+}$Calcd for $\mathrm{C}_{16} \mathrm{H}_{15} \mathrm{~N}_{2} \mathrm{O}_{4} 299.1065$; Found 299.1067.

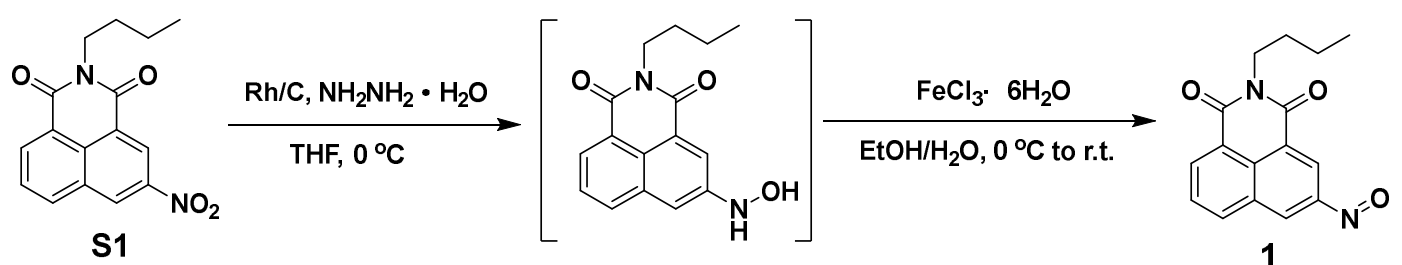

Synthesis of 2-butyl-5-nitroso-1 $H$-benzo[de] isoquinoline-1,3(2H)-dione $(1)^{1}$

To a solution of $\mathbf{S 1}(1.18 \mathrm{~g}, 3.96 \mathrm{mmol})$ in THF $(20 \mathrm{~mL})$ was added $\mathrm{Rh} / \mathrm{C}(200 \mathrm{mg}$, $5 \%)$ and hydrazine hydrate $(450 \mathrm{mg}, 9.1 \mathrm{mmol})$ at $0{ }^{\circ} \mathrm{C}$. The reaction mixture was stirred at $0{ }^{\circ} \mathrm{C}$ for $1.5 \mathrm{~h}$. After filtration, the filtrate was concentrated, redissolved in 
EtOH $\left(10 \mathrm{~mL}\right.$ ), and added to an ice cooled aqueous $\mathrm{FeCl}_{3}$ solution (hexaaquo complex, $2.1 \mathrm{~g}, 7.92 \mathrm{mmol}$ in $20 \mathrm{~mL}$ ). The reaction mixture was stirred at r.t. for 30 min, then solvent was evaporated and the resulting residue was extracted with EtOAc $(3 \times 8 \mathrm{~mL})$. The combined organic layers were washed with brine, dried over anhydrous $\mathrm{Na}_{2} \mathrm{SO}_{4}$, filtered, and concentrated. The residue was purified by silica gel flash column chromatography $(\mathrm{PE} / \mathrm{EtOAc}=2: 1)$ to afford the titled compound as green solid (612 mg, $55 \%$ yield for 2 steps). m.p.: $172-175^{\circ} \mathrm{C} ;{ }^{1} \mathrm{H} \mathrm{NMR}\left(400 \mathrm{MHz}, \mathrm{CDCl}_{3}\right)$ $\delta 9.58(\mathrm{~s}, 1 \mathrm{H}), 8.79(\mathrm{~d}, J=7.3 \mathrm{~Hz}, 1 \mathrm{H}), 8.64(\mathrm{~d}, J=8.2 \mathrm{~Hz}, 1 \mathrm{H}), 8.28(\mathrm{~s}, 1 \mathrm{H}), 7.96(\mathrm{t}$, $J=7.8 \mathrm{~Hz}, 1 \mathrm{H}), 4.22-4.14(\mathrm{~m}, 2 \mathrm{H}), 1.75-1.67(\mathrm{~m}, 2 \mathrm{H}), 1.48-1.40(\mathrm{~m}, 2 \mathrm{H}), 0.98(\mathrm{t}$, $J=7.3 \mathrm{~Hz}, 3 \mathrm{H}) ;{ }^{13} \mathrm{C}$ NMR $\left(101 \mathrm{MHz}, \mathrm{CDCl}_{3}\right) \delta 163.4,162.3,136.8,136.3,134.6$, 131.9, 131.0, 130.6, 128.9, 124.2, 123.5, 115.4, 40.7, 30.2, 20.4, 13.9; HRMS (ESI) $\mathrm{m} / \mathrm{z}:[\mathrm{M}+\mathrm{H}]^{+}$Calcd for $\mathrm{C}_{16} \mathrm{H}_{15} \mathrm{~N}_{2} \mathrm{O}_{3}$ 283.1077; Found 283.1074.

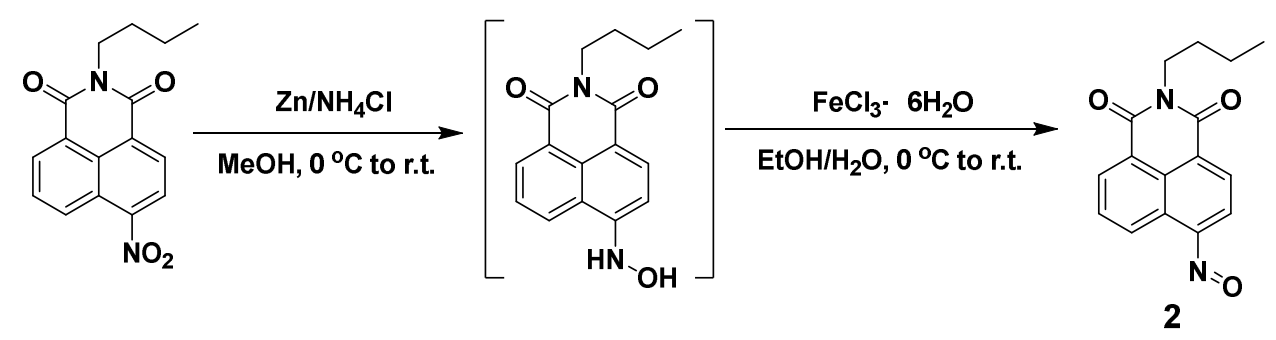

Synthesis of 2-butyl-6-nitroso- $1 H$-benzo[de]isoquinoline-1,3(2H)-dione $(2)^{2}$

To a solution of 4-nitro-1,8-naphthalimide ${ }^{3}(345 \mathrm{mg}, 1.16 \mathrm{mmol})$ in $\mathrm{MeOH}(10 \mathrm{~mL})$ was added zinc dust $(165 \mathrm{mg}, 2.54 \mathrm{mmol})$ and $\mathrm{NH}_{4} \mathrm{Cl}(74.5 \mathrm{mg}, 1.39 \mathrm{mmol})$ at $0^{\circ} \mathrm{C}$. The reaction mixture was stirred at r.t. for $1 \mathrm{~h}$. After filtration, the filtrate was concentrated, redissolved in $\mathrm{EtOH}(5 \mathrm{~mL})$, and added to an ice cooled aqueous $\mathrm{FeCl}_{3}$ solution (hexaaquo complex, $627 \mathrm{mg}, 2.32 \mathrm{mmol}$ in $10 \mathrm{~mL}$ ). The reaction mixture was stirred at r.t. for $30 \mathrm{~min}$, then solvent was evaporated and the resulting residue was extracted with EtOAc $(3 \times 5 \mathrm{~mL})$. The combined organic layers were washed with brine, dried over anhydrous $\mathrm{Na}_{2} \mathrm{SO}_{4}$, filtered, and concentrated. The residue was purified by silica gel flash column chromatography $(\mathrm{PE} / \mathrm{EtOAc}=20: 1)$ to afford the titled compound as yellow solid $\left(172 \mathrm{mg}, 53 \%\right.$ yield for 2 steps). m.p.: $176-178{ }^{\circ} \mathrm{C} ;{ }^{1} \mathrm{H}$ NMR $\left(400 \mathrm{MHz}, \mathrm{CDCl}_{3}\right) \delta 10.16(\mathrm{~d}, J=9.6 \mathrm{~Hz}, 1 \mathrm{H}), 8.78(\mathrm{~d}, J=7.3 \mathrm{~Hz}, 1 \mathrm{H}), 8.62(\mathrm{~d}$, $J=7.9 \mathrm{~Hz}, 1 \mathrm{H}), 8.28-8.19(\mathrm{~m}, 1 \mathrm{H}), 6.62(\mathrm{~d}, J=7.9 \mathrm{~Hz}, 1 \mathrm{H}), 4.24-4.18(\mathrm{~m}, 2 \mathrm{H})$, $1.81-1.68(\mathrm{~m}, 2 \mathrm{H}), 1.51-1.42(\mathrm{~m}, 2 \mathrm{H}), 0.99(\mathrm{t}, J=7.3 \mathrm{~Hz}, 3 \mathrm{H}) ;{ }^{13} \mathrm{C}$ NMR $(101$ $\left.\mathrm{MHz}, \mathrm{CDCl}_{3}\right) \delta 164.1,163.1,156.1,131.9,130.9,130.5,130.5,130.2,129.1,128.1$, 122.8, 105.2, 40.7, 30.2, 20.4, 13.9; HRMS (ESI) $\mathrm{m} / \mathrm{z}$ : $[\mathrm{M}+\mathrm{H}]^{+}$Calcd for $\mathrm{C}_{16} \mathrm{H}_{15} \mathrm{~N}_{2} \mathrm{O}_{3} 283.1077$; Found 283.1075.

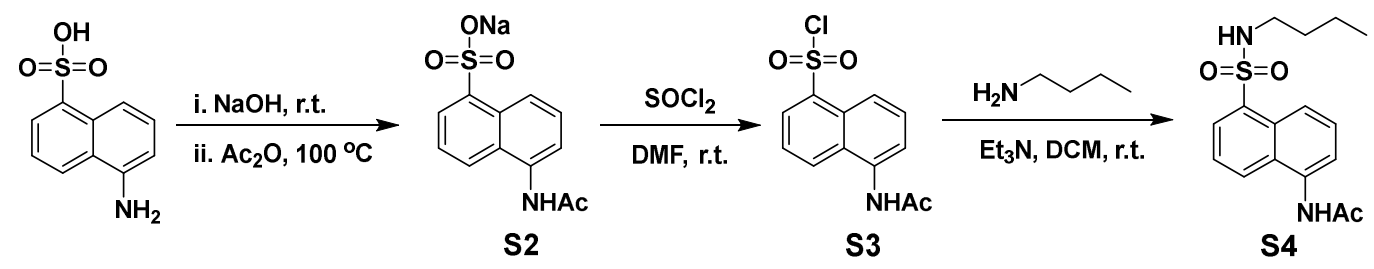




\section{Synthesis of $N$-(5-(N-butylsulfamoyl)naphthalen-1-yl)acetamide (S4) ${ }^{4}$}

To a solution of 5-aminonaphthalene-1-sulfonic acid (3.9 g, $17.5 \mathrm{mmol})$ in $\mathrm{H}_{2} \mathrm{O}$ (55 $\mathrm{mL}$ ) was added $5 \mathrm{M}$ aqueous $\mathrm{NaOH}$ solution (700 mg, $17.5 \mathrm{mmol}$ in $3.5 \mathrm{~mL}$ ). The reaction mixture was stirred at r.t. for $10 \mathrm{~min}$, then concentrated. The residue was suspended in $\mathrm{Ac}_{2} \mathrm{O}(18 \mathrm{~mL})$ and stirred at $100{ }^{\circ} \mathrm{C}$ using an oil bath for $2 \mathrm{~h}$, then concentrated to afford crude sodium 5-acetamidonaphthalene-1-sulfonate (S2) used directly for next step.

To a solution of crude $\mathbf{S} 2$ in DMF $(20 \mathrm{~mL})$ was added $\mathrm{SOCl}_{2}(8.3 \mathrm{~g}, 70 \mathrm{mmol})$ at 0 ${ }^{\circ} \mathrm{C}$. The reaction mixture was stirred at r.t. for $1 \mathrm{~h}$, then quenched with $\mathrm{H}_{2} \mathrm{O}(10 \mathrm{~mL})$ and extracted with EtOAc $(3 \times 10 \mathrm{~mL})$. The combined organic layers were washed with $\mathrm{H}_{2} \mathrm{O}$, brine, dried over anhydrous $\mathrm{Na}_{2} \mathrm{SO}_{4}$, filtered, and concentrated to afford crude 5-acetamidonaphthalene-1-sulfonyl chloride (S3) used directly for next step.

To a solution of crude $\mathbf{S 3}$ in DCM $(20 \mathrm{~mL})$ was added $\mathrm{Et}_{3} \mathrm{~N}(3.5 \mathrm{~g}, 35 \mathrm{mmol})$ and $n$-butylamine (1.9 g, $26.3 \mathrm{mmol})$. The reaction mixture was stirred at r.t. for $1 \mathrm{~h}$, then concentrated. The residue was purified by silica gel flash column chromatography $(\mathrm{PE} / \mathrm{EtOAc}=2: 1)$ to afford the titled compound as white solid $(1.9 \mathrm{~g}, 34 \%$ yield for three steps). m.p.: 206-209 ${ }^{\circ} \mathrm{C} ;{ }^{1} \mathrm{H}$ NMR $\left(400 \mathrm{MHz}, \mathrm{CDCl}_{3}\right) \delta 8.44(\mathrm{~d}, J=8.7 \mathrm{~Hz}, 1 \mathrm{H})$, $8.18(\mathrm{~d}, J=7.3 \mathrm{~Hz}, 1 \mathrm{H}), 8.04(\mathrm{~d}, J=8.5 \mathrm{~Hz}, 1 \mathrm{H}), 7.76-7.68(\mathrm{~m}, 2 \mathrm{H}), 7.54$ (t, $J=8.1$ $\mathrm{Hz}, 1 \mathrm{H}), 7.43(\mathrm{t}, J=7.9 \mathrm{~Hz}, 1 \mathrm{H}), 4.61(\mathrm{t}, J=5.8 \mathrm{~Hz}, 1 \mathrm{H}), 2.87(\mathrm{q}, J=6.7 \mathrm{~Hz}, 2 \mathrm{H})$, $2.33(\mathrm{~s}, 3 \mathrm{H}), 1.41-1.32(\mathrm{~m}, 2 \mathrm{H}), 1.25-1.15(\mathrm{~m}, 2 \mathrm{H}), 0.77(\mathrm{t}, J=7.3 \mathrm{~Hz}, 3 \mathrm{H}) ;{ }^{13} \mathrm{C}$ NMR (101 MHz, CD $\left.{ }_{3} \mathrm{OD}\right) \delta 172.3,136.8,134.6,130.6,129.8,129.6,128.7,127.9$, 124.9, 124.7, 123.9, 42.9, 32.1, 22.6, 20.0, 13.1; HRMS (ESI) m/z: $[\mathrm{M}+\mathrm{H}]^{+}$Calcd for $\mathrm{C}_{16} \mathrm{H}_{21} \mathrm{~N}_{2} \mathrm{O}_{3} \mathrm{~S}$ 321.1267; Found 321.1273.

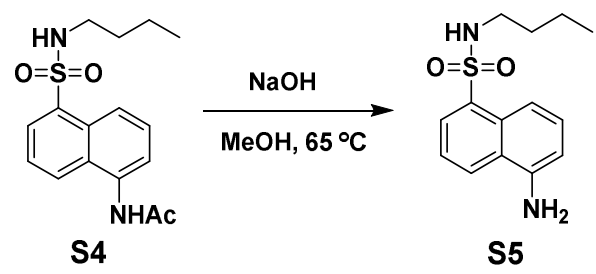

\section{Synthesis of 5-amino- $\boldsymbol{N}$-butylnaphthalene-1-sulfonamide (S5)}

To a solution of $\mathbf{S 4}(1.9 \mathrm{~g}, 5.9 \mathrm{mmol})$ in $\mathrm{MeOH}(10 \mathrm{~mL})$ was added $5 \mathrm{M}$ aqueous $\mathrm{NaOH}$ solution ( $3 \mathrm{~g}, 75 \mathrm{mmol}$ in $15 \mathrm{~mL}$ ). The reaction mixture was stirred at $65{ }^{\circ} \mathrm{C}$ using an oil bath for $16 \mathrm{~h}$, then concentrated. The residue was purified by silica gel flash column chromatography $(\mathrm{PE} / \mathrm{EtOAc}=3: 2)$ to afford the titled compound as light green solid (1.55 g, 95\% yield). m.p.: $170-172{ }^{\circ} \mathrm{C} ;{ }^{1} \mathrm{H} \mathrm{NMR}\left(400 \mathrm{MHz}, \mathrm{CDCl}_{3}\right) \delta$ $8.25(\mathrm{~d}, J=7.3 \mathrm{~Hz}, 1 \mathrm{H}), 8.09$ (d, $J=8.5 \mathrm{~Hz}, 1 \mathrm{H}), 8.04$ (d, $J=8.6 \mathrm{~Hz}, 1 \mathrm{H}), 7.50-$ $7.42(\mathrm{~m}, 2 \mathrm{H}), 6.88(\mathrm{~d}, J=7.5 \mathrm{~Hz}, 1 \mathrm{H}), 4.61(\mathrm{t}, J=6.2 \mathrm{~Hz}, 1 \mathrm{H}), 4.36-4.20(\mathrm{~m}, 2 \mathrm{H})$, $2.85(\mathrm{q}, J=6.9 \mathrm{~Hz}, 2 \mathrm{H}), 1.39-1.28(\mathrm{~m}, 2 \mathrm{H}), 1.23-1.12(\mathrm{~m}, 2 \mathrm{H}), 0.74(\mathrm{t}, J=7.3 \mathrm{~Hz}$, $3 \mathrm{H}) ;{ }^{13} \mathrm{C}$ NMR $\left(101 \mathrm{MHz}, \mathrm{CDCl}_{3}\right) \delta 143.4,134.8,129.9,129.3,129.1,127.0,124.5$, 122.9, 114.8, 111.0, 43.1, 31.6, 19.7, 13.5; HRMS (ESI) m/z: $[\mathrm{M}+\mathrm{H}]^{+}$Calcd for $\mathrm{C}_{14} \mathrm{H}_{19} \mathrm{~N}_{2} \mathrm{O}_{2} \mathrm{~S} 279.1162$; Found 279.1172. 


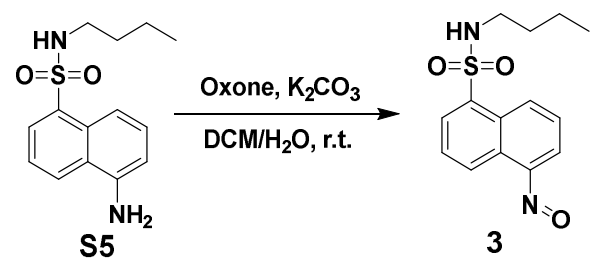

Synthesis of $\boldsymbol{N}$-butyl-5-nitrosonaphthalene-1-sulfonamide (3)

To a solution of $\mathbf{S 5}(500 \mathrm{mg}, 1.8 \mathrm{mmol})$ in $\mathrm{DCM} / \mathrm{H}_{2} \mathrm{O}(25 \mathrm{~mL}, 1: 4)$ was added Oxone (2.34 g, $7.6 \mathrm{mmol})$ and $\mathrm{K}_{2} \mathrm{CO}_{3}(248 \mathrm{mg}, 1.8 \mathrm{mmol})$. The reaction mixture was stirred at r.t. for $4 \mathrm{~h}$, then extracted with DCM $(3 \times 10 \mathrm{~mL})$. The combined organic layers were washed with brine, dried over anhydrous $\mathrm{Na}_{2} \mathrm{SO}_{4}$, filtered, and concentrated. The residue was purified by silica gel flash column chromatography $(\mathrm{PE} / \mathrm{EtOAc}=4: 1)$ to afford the titled compound as white solid $(124 \mathrm{mg}, 24 \%$ yield $)$. m.p.: $186-188^{\circ} \mathrm{C} ;{ }^{1} \mathrm{H}$ NMR $\left(400 \mathrm{MHz}, \mathrm{CDCl}_{3}\right) \delta 10.30(\mathrm{~d}, J=8.6 \mathrm{~Hz}, 1 \mathrm{H}), 9.16(\mathrm{~d}, J$ $=8.5 \mathrm{~Hz}, 1 \mathrm{H}), 8.49(\mathrm{~d}, J=7.3 \mathrm{~Hz}, 1 \mathrm{H}), 8.12-8.01(\mathrm{~m}, 1 \mathrm{H}), 7.74-7.65(\mathrm{~m}, 1 \mathrm{H})$, $6.48(\mathrm{~d}, J=7.5 \mathrm{~Hz}, 1 \mathrm{H}), 4.78(\mathrm{t}, J=6.1 \mathrm{~Hz}, 1 \mathrm{H}), 3.02(\mathrm{q}, J=6.9 \mathrm{~Hz}, 2 \mathrm{H}), 1.46-1.37$ (m, 2H), $1.27-1.18(\mathrm{~m}, 2 \mathrm{H}), 0.77(\mathrm{t}, J=7.3 \mathrm{~Hz}, 3 \mathrm{H}) ;{ }^{13} \mathrm{C} \mathrm{NMR}\left(101 \mathrm{MHz}, \mathrm{CDCl}_{3}\right) \delta$ 158.8, 135.8, 133.5, 132.8, 130.5, 130.0, 129.6, 127.9, 126.9, 104.4, 43.2, 31.7, 19.7, 13.5; HRMS (ESI) m/z: [M + H $]^{+}$Calcd for $\mathrm{C}_{14} \mathrm{H}_{17} \mathrm{~N}_{2} \mathrm{O}_{3} \mathrm{~S}$ 293.0954; Found 293.0945.

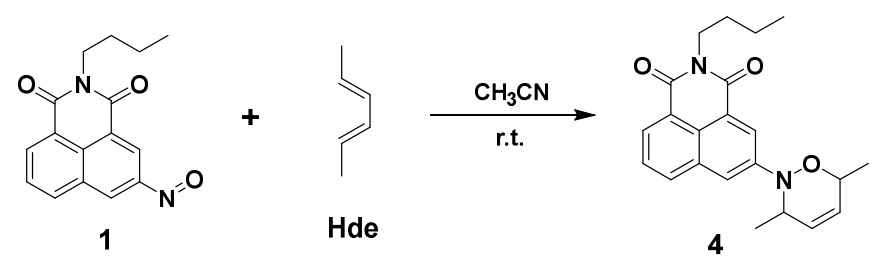

Synthesis of 2-butyl-5-(3,6-dimethyl-3,6-dihydro-2H-1,2-oxazin-2-yl)-1H-benzo [de]isoquinoline-1,3(2H)-dione (4)

To a solution of $\mathbf{1}(300 \mathrm{mg}, 1.06 \mathrm{mmol})$ in $\mathrm{CH}_{3} \mathrm{CN}(25 \mathrm{~mL})$ was added (2E,4E)-hexa-2,4-diene (Hde, $261 \mathrm{mg}, 3.18 \mathrm{mmol}$ ). The reaction mixture was stirred at r.t. for $30 \mathrm{~min}$, then concentrated. The residue was purified by silica gel flash column chromatography $(\mathrm{PE} / \mathrm{EtOAc}=10: 1)$ to afford the titled compound as yellow solid (340 mg, 88\% yield). m.p.: $181-183{ }^{\circ} \mathrm{C}$; ${ }^{1} \mathrm{H}$ NMR $\left(600 \mathrm{MHz}, \mathrm{CDCl}_{3}\right) \delta 8.39$ (d, $J$ $=7.2 \mathrm{~Hz}, 1 \mathrm{H}), 8.27(\mathrm{~d}, J=2.3 \mathrm{~Hz}, 1 \mathrm{H}), 8.06(\mathrm{~d}, J=8.1 \mathrm{~Hz}, 1 \mathrm{H}), 7.78(\mathrm{~d}, J=2.3 \mathrm{~Hz}$, $1 \mathrm{H}), 7.68-7.63(\mathrm{~m}, 1 \mathrm{H}), 6.02-5.96(\mathrm{~m}, 1 \mathrm{H}), 5.83(\mathrm{~d}, J=10.0 \mathrm{~Hz}, 1 \mathrm{H}), 4.81-4.74$ (m, 1H), $4.46-4.38(\mathrm{~m}, 1 \mathrm{H}), 4.21-4.13(\mathrm{~m}, 2 \mathrm{H}), 1.75-1.67(\mathrm{~m}, 2 \mathrm{H}), 1.49-1.41$ $(\mathrm{m}, 2 \mathrm{H}), 1.39$ (d, $J=6.8 \mathrm{~Hz}, 3 \mathrm{H}), 1.16(\mathrm{~d}, J=6.5 \mathrm{~Hz}, 3 \mathrm{H}), 0.98(\mathrm{t}, J=7.4 \mathrm{~Hz}, 3 \mathrm{H})$; ${ }^{13} \mathrm{C}$ NMR $\left(151 \mathrm{MHz}, \mathrm{CDCl}_{3}\right) \delta 164.4,164.3,147.4,132.9,132.8,130.0,128.4,128.4$, 127.2, 123.6, 123.3, 122.5, 121.1, 115.9, 74.3, 54.3, 40.3, 30.3, 20.4, 18.9, 14.6, 13.9; HRMS (ESI) m/z: $[\mathrm{M}+\mathrm{H}]^{+}$Calcd for $\mathrm{C}_{22} \mathrm{H}_{25} \mathrm{~N}_{2} \mathrm{O}_{3} 365.1860$; Found 365.1872. 


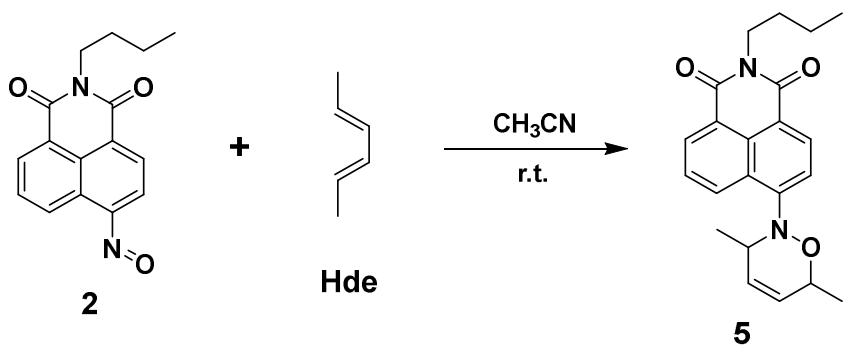

Synthesis of 2-butyl-6-(3,6-dimethyl-3,6-dihydro-2H-1,2-oxazin-2-yl)-1H-benzo [de] isoquinoline-1,3(2H)-dione (5)

To a solution of $2(25 \mathrm{mg}, 0.089 \mathrm{mmol})$ in $\mathrm{CH}_{3} \mathrm{CN}(3 \mathrm{~mL})$ was added (2E,4E)-hexa-2,4-diene (Hde, $22 \mathrm{mg}, 0.27 \mathrm{mmol}$ ). The reaction mixture was stirred at r.t. for $30 \mathrm{~min}$, then concentrated. The residue was purified by silica gel flash column chromatography $(\mathrm{PE} / \mathrm{EtOAc}=10: 1)$ to afford the titled compound as yellow solid (29 mg, 91\% yield). m.p.: $136-138^{\circ} \mathrm{C}$; ${ }^{1} \mathrm{H}$ NMR $\left(600 \mathrm{MHz}, \mathrm{CDCl}_{3}\right) \delta 8.61-8.54(\mathrm{~m}, 2 \mathrm{H})$, $8.42(\mathrm{~d}, J=8.4 \mathrm{~Hz}, 1 \mathrm{H}), 7.70-7.64(\mathrm{~m}, 2 \mathrm{H}), 6.01-5.95(\mathrm{~m}, 1 \mathrm{H}), 5.85(\mathrm{~d}, J=10.0$ $\mathrm{Hz}, 1 \mathrm{H}), 4.77-4.70(\mathrm{~m}, 1 \mathrm{H}), 4.26-4.20(\mathrm{~m}, 1 \mathrm{H}), 4.20-4.14(\mathrm{~m}, 2 \mathrm{H}), 1.76-1.69$ (m, 2H), $1.49-1.41(\mathrm{~m}, 2 \mathrm{H}), 1.34(\mathrm{~d}, J=6.7 \mathrm{~Hz}, 3 \mathrm{H}), 1.02-0.96(\mathrm{~m}, 6 \mathrm{H}) ;{ }^{13} \mathrm{C} \mathrm{NMR}$ $\left(151 \mathrm{MHz}, \mathrm{CDCl}_{3}\right) \delta 164.4,164.1,151.7,132.0,131.1,130.1,129.2,128.9,128.6$, $125.8,124.3,123.0,117.9,116.3,74.1,58.1,40.2,30.3,20.4,19.0,15.5,13.9$; HRMS (ESI) m/z: $[\mathrm{M}+\mathrm{H}]^{+}$Calcd for $\mathrm{C}_{22} \mathrm{H}_{25} \mathrm{~N}_{2} \mathrm{O}_{3} 365.1860$; Found 365.1868.

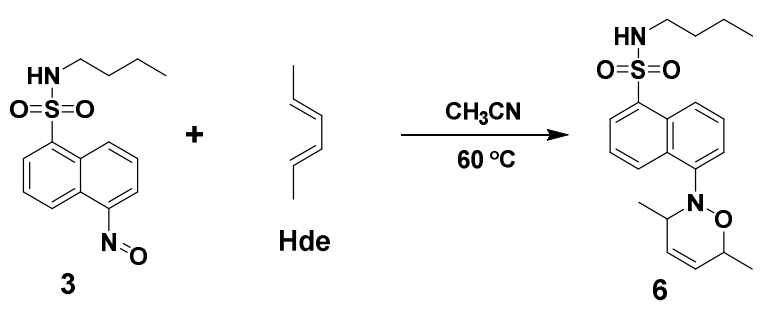

Synthesis of $\quad \mathrm{N}$-butyl-5-(3,6-dimethyl-3,6-dihydro-2 $H$-1,2-oxazin-2-yl) naphthalene-1-sulfonamide (6)

To a solution of $3(40 \mathrm{mg}, 0.137 \mathrm{mmol})$ in $\mathrm{CH}_{3} \mathrm{CN}(4 \mathrm{~mL})$ was added (2E,4E)-hexa-2,4-diene (Hde, $34 \mathrm{mg}, 0.41 \mathrm{mmol}$ ). The reaction mixture was stirred at $60{ }^{\circ} \mathrm{C}$ using an oil bath for $1 \mathrm{~h}$, then concentrated. The residue was purified by silica gel flash column chromatography $(\mathrm{PE} / \mathrm{EtOAc}=7: 1)$ to afford the titled compound as yellow oil (43 mg, 84\% yield). ${ }^{1} \mathrm{H}$ NMR $\left(600 \mathrm{MHz}, \mathrm{CDCl}_{3}\right) \delta 8.54(\mathrm{~d}, J=8.5 \mathrm{~Hz}, 1 \mathrm{H})$, $8.49-8.43(\mathrm{~m}, 1 \mathrm{H}), 8.26(\mathrm{~d}, J=7.2 \mathrm{~Hz}, 1 \mathrm{H}), 7.66-7.61(\mathrm{~m}, 2 \mathrm{H}), 7.55-7.48(\mathrm{~m}$, $1 \mathrm{H}), 5.98-5.93(\mathrm{~m}, 1 \mathrm{H}), 5.87-5.82(\mathrm{~m}, 1 \mathrm{H}), 4.79-4.72(\mathrm{~m}, 1 \mathrm{H}), 4.65-4.58(\mathrm{~m}$, $1 \mathrm{H}), 4.03-3.95(\mathrm{~m}, 1 \mathrm{H}), 2.96-2.82(\mathrm{~m}, 2 \mathrm{H}), 1.37-1.31(\mathrm{~m}, 5 \mathrm{H}), 1.21-1.13(\mathrm{~m}$, $2 \mathrm{H}), 0.96(\mathrm{~d}, J=6.5 \mathrm{~Hz}, 3 \mathrm{H}), 0.73(\mathrm{t}, J=7.4 \mathrm{~Hz}, 3 \mathrm{H}) ;{ }^{13} \mathrm{C} \mathrm{NMR}\left(151 \mathrm{MHz}, \mathrm{CDCl}_{3}\right) \delta$ 146.2, 134.6, 130.0, 129.8, 129.7, 129.2, 129.1, 128.9, 128.1, 123.6, 121.1, 118.4, 73.5, 57.4, 43.0, 31.5, 19.6, 19.3, 15.9, 13.4; HRMS (ESI) m/z: $[\mathrm{M}+\mathrm{H}]^{+}$Calcd for $\mathrm{C}_{20} \mathrm{H}_{27} \mathrm{~N}_{2} \mathrm{O}_{3} \mathrm{~S}$ 375.1737; Found 375.1751. 


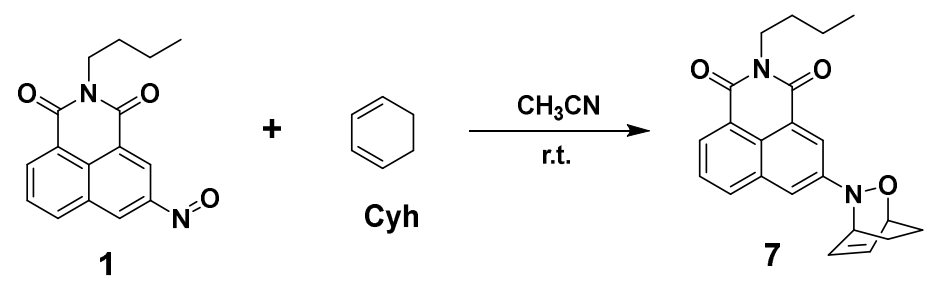

Synthesis of 5-(2-oxa-3-azabicyclo[2.2.2]oct-5-en-3-yl)-2-butyl-1H-benzo[de] isoquinoline-1,3(2H)-dione (7)

To a solution of $1(300 \mathrm{mg}, 1.06 \mathrm{mmol})$ in $\mathrm{CH}_{3} \mathrm{CN}(25 \mathrm{~mL})$ was added cyclohexa-1,3-diene (Cyh, $170 \mathrm{mg}, 2.12 \mathrm{mmol}$ ). The reaction mixture was stirred at r.t. for $5 \mathrm{~min}$, then concentrated. The residue was purified by silica gel flash column chromatography $(\mathrm{PE} / \mathrm{EtOAc}=6: 1)$ to afford the titled compound as yellow solid (350 mg, 91\% yield). m.p.: $191-193{ }^{\circ} \mathrm{C} ;{ }^{1} \mathrm{H}$ NMR $\left(400 \mathrm{MHz}, \mathrm{CDCl}_{3}\right) \delta 8.40(\mathrm{~d}, J=7.3 \mathrm{~Hz}$, $1 \mathrm{H}), 8.28(\mathrm{~d}, J=2.3 \mathrm{~Hz}, 1 \mathrm{H}), 8.03(\mathrm{~d}, J=8.2 \mathrm{~Hz}, 1 \mathrm{H}), 7.71(\mathrm{~d}, J=2.3 \mathrm{~Hz}, 1 \mathrm{H}), 7.67$ - $7.62(\mathrm{~m}, 1 \mathrm{H}), 6.66-6.58(\mathrm{~m}, 1 \mathrm{H}), 6.17-6.10(\mathrm{~m}, 1 \mathrm{H}), 4.88-4.83(\mathrm{~m}, 1 \mathrm{H}), 4.76-$ $4.70(\mathrm{~m}, 1 \mathrm{H}), 4.20-4.13(\mathrm{~m}, 2 \mathrm{H}), 2.44-2.26(\mathrm{~m}, 2 \mathrm{H}), 1.76-1.60(\mathrm{~m}, 4 \mathrm{H}), 1.52-$ $1.37(\mathrm{~m}, 4 \mathrm{H}), 0.97(\mathrm{t}, J=7.3 \mathrm{~Hz}, 3 \mathrm{H}) ;{ }^{13} \mathrm{C} \mathrm{NMR}\left(101 \mathrm{MHz}, \mathrm{CDCl}_{3}\right) \delta 164.4,164.3$, $151.1,133.1,132.7,131.8,129.7,128.8,127.3,124.4,123.1,122.7,122.6,119.0$, 70.0, 56.4, 40.3, 30.3, 24.0, 21.3, 20.5, 13.9; HRMS (ESI) m/z: $[\mathrm{M}+\mathrm{H}]^{+}$Calcd for $\mathrm{C}_{22} \mathrm{H}_{23} \mathrm{~N}_{2} \mathrm{O}_{3} 363.1703$; Found 363.1713.

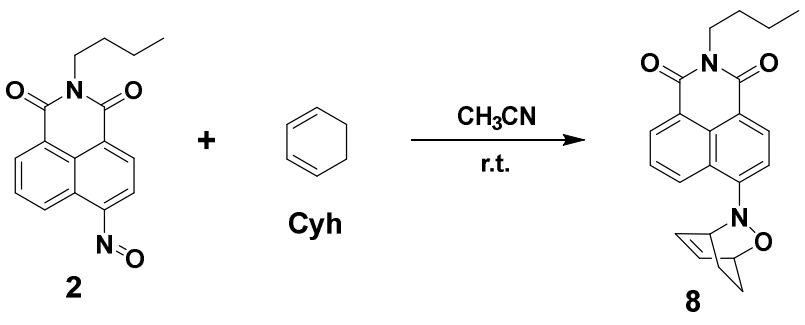

Synthesis of 6-(2-oxa-3-azabicyclo[2.2.2]oct-5-en-3-yl)-2-butyl-1H-benzo[de] isoquinoline-1,3(2H)-dione $(8)$

To a solution of $2(30 \mathrm{mg}, 0.11 \mathrm{mmol})$ in $\mathrm{CH}_{3} \mathrm{CN}(3 \mathrm{~mL})$ was added cyclohexa-1,3-diene (Cyh, $26 \mathrm{mg}, 0.32 \mathrm{mmol}$ ). The reaction mixture was stirred at r.t. for $5 \mathrm{~min}$, then concentrated. The residue was purified by silica gel flash column chromatography $(\mathrm{PE} / \mathrm{EtOAc}=5: 1)$ to afford the titled compound as brown solid (36 mg, 93\% yield). m.p.: $179-182{ }^{\circ} \mathrm{C} ;{ }^{1} \mathrm{H}$ NMR (400 MHz, $\left.\mathrm{CDCl}_{3}\right) \delta 8.58(\mathrm{~d}, J=7.3 \mathrm{~Hz}$, $1 \mathrm{H}), 8.46-8.42(\mathrm{~m}, 2 \mathrm{H}), 7.73-7.68(\mathrm{~m}, 1 \mathrm{H}), 7.38(\mathrm{~d}, J=8.2 \mathrm{~Hz}, 1 \mathrm{H}), 6.80-6.72$ (m, 1H), $6.00-5.92(\mathrm{~m}, 1 \mathrm{H}), 4.92-4.87(\mathrm{~m}, 1 \mathrm{H}), 4.55-4.46(\mathrm{~m}, 1 \mathrm{H}), 4.20-4.11$ $(\mathrm{m}, 2 \mathrm{H}), 2.63-2.50(\mathrm{~m}, 1 \mathrm{H}), 2.46-2.34(\mathrm{~m}, 1 \mathrm{H}), 1.75-1.67(\mathrm{~m}, 2 \mathrm{H}), 1.66-1.58$ $(\mathrm{m}, 1 \mathrm{H}), 1.56-1.47(\mathrm{~m}, 1 \mathrm{H}), 1.47-1.39(\mathrm{~m}, 2 \mathrm{H}), 0.96(\mathrm{t}, J=7.3 \mathrm{~Hz}, 3 \mathrm{H}) ;{ }^{13} \mathrm{C} \mathrm{NMR}$ $\left(101 \mathrm{MHz} \mathrm{CDCl}_{3}\right) \delta 164.5,164.1,153.7,132.0,131.8,131.0,129.3,129.2,128.7$, $126.0,124.2,123.1,117.8,117.1,70.0,58.5,40.2,30.3,23.6,22.0,20.5,13.9$; HRMS (ESI) m/z: [M+ H] ${ }^{+}$Calcd for $\mathrm{C}_{22} \mathrm{H}_{23} \mathrm{~N}_{2} \mathrm{O}_{3} 363.1703$; Found 363.1697. 


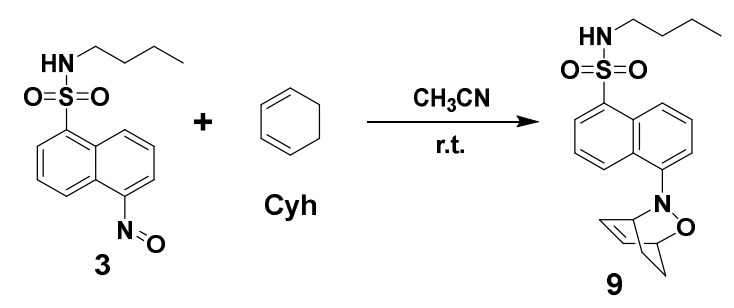

Synthesis of 5-(2-oxa-3-azabicyclo[2.2.2]oct-5-en-3-yl)- $N$-butylnaphthalene -1-sulfonamide (9)

To a solution of $3(20 \mathrm{mg}, 0.068 \mathrm{mmol})$ in $\mathrm{CH}_{3} \mathrm{CN}(2 \mathrm{~mL})$ was added cyclohexa-1,3-diene (Cyh, $16 \mathrm{mg}, 0.21 \mathrm{mmol}$ ). The reaction mixture was stirred at r.t. for $5 \mathrm{~min}$, then concentrated. The residue was purified by silica gel flash column chromatography $(\mathrm{PE} / \mathrm{EtOAc}=2: 1)$ to afford the titled compound as yellow solid (23 mg, 90\% yield). m.p.: $205-207^{\circ} \mathrm{C} ;{ }^{1} \mathrm{H}$ NMR $\left(400 \mathrm{MHz}, \mathrm{CDCl}_{3}\right) \delta 8.49(\mathrm{~d}, J=8.5 \mathrm{~Hz}$, $1 \mathrm{H}), 8.31(\mathrm{~d}, J=8.6 \mathrm{~Hz}, 1 \mathrm{H}), 8.24(\mathrm{~d}, J=7.3 \mathrm{~Hz}, 1 \mathrm{H}), 7.56-7.47(\mathrm{~m}, 2 \mathrm{H}), 7.30(\mathrm{~d}, J$ $=7.5 \mathrm{~Hz}, 1 \mathrm{H}), 6.80-6.74(\mathrm{~m}, 1 \mathrm{H}), 5.98-5.92(\mathrm{~m}, 1 \mathrm{H}), 4.86-4.80(\mathrm{~m}, 1 \mathrm{H}), 4.54(\mathrm{t}$, $J=6.1 \mathrm{~Hz}, 1 \mathrm{H}), 4.28-4.22(\mathrm{~m}, 1 \mathrm{H}), 2.99-2.79(\mathrm{~m}, 2 \mathrm{H}), 2.58-2.47(\mathrm{~m}, 1 \mathrm{H}), 2.43-$ $2.32(\mathrm{~m}, 1 \mathrm{H}), 1.67-1.43(\mathrm{~m}, 3 \mathrm{H}), 1.25-1.13(\mathrm{~m}, 3 \mathrm{H}), 0.72(\mathrm{t}, J=7.3 \mathrm{~Hz}, 3 \mathrm{H}) ;{ }^{13} \mathrm{C}$ NMR $\left(101 \mathrm{MHz}, \mathrm{CDCl}_{3}\right) \delta 148.0,134.9,132.2,129.4,129.1,129.0,128.9,127.9$, 127.6, 123.6, 120.0, 118.0, 69.6, 57.5, 43.1, 31.6, 23.7, 22.2, 19.6, 13.5; HRMS (ESI) $\mathrm{m} / \mathrm{z}:[\mathrm{M}+\mathrm{H}]^{+}$Calcd for $\mathrm{C}_{20} \mathrm{H}_{25} \mathrm{~N}_{2} \mathrm{O}_{3} \mathrm{~S}$ 373.1580; Found 373.1582 .

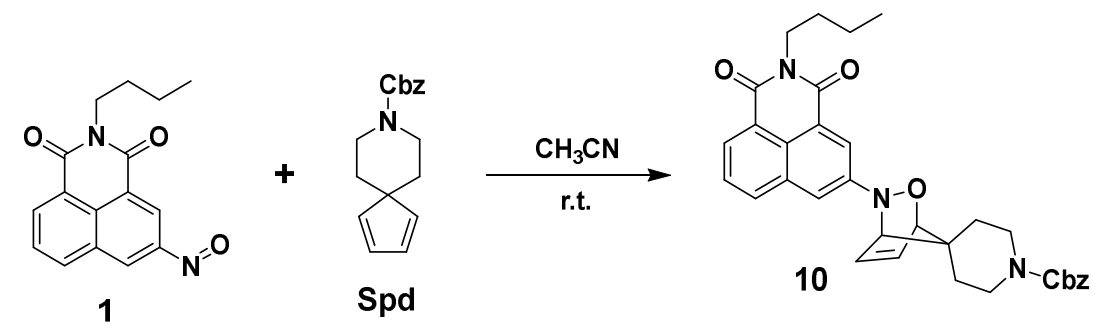

Synthesis of benzyl 3-(2-butyl-1,3-dioxo-2,3-dihydro-1 $H$-benzo[de]isoquinolin-5yl)-2-oxa-3-azaspiro[bicyclo[2.2.1] hept [5]ene-7,4'-piperidine]-1'-carboxylate (10)

To a solution of 1 (35 mg, $0.124 \mathrm{mmol})$ in $\mathrm{CH}_{3} \mathrm{CN}(3 \mathrm{~mL})$ was added benzyl 8-azaspiro[4.5]deca-1,3-diene-8-carboxylate ${ }^{6}$ (Spd, $67 \mathrm{mg}, 0.25 \mathrm{mmol}$ ). The reaction mixture was stirred at r.t. for $5 \mathrm{~min}$, then concentrated. The residue was purified by silica gel flash column chromatography $(\mathrm{PE} / \mathrm{EtOAc}=3: 1)$ to afford the titled compound as yellow solid (61 mg, 90\% yield). m.p.: 210-213 ${ }^{\circ} \mathrm{C} ;{ }^{1} \mathrm{H}$ NMR (400 MHz, $\left.\mathrm{CDCl}_{3}\right) \delta 8.39(\mathrm{~d}, J=7.3 \mathrm{~Hz}, 1 \mathrm{H}), 8.21(\mathrm{~d}, J=2.2 \mathrm{~Hz}, 1 \mathrm{H}), 7.99(\mathrm{~d}, J=8.3 \mathrm{~Hz}, 1 \mathrm{H})$, $7.69-7.61(\mathrm{~m}, 1 \mathrm{H}), 7.57(\mathrm{~d}, J=2.2 \mathrm{~Hz}, 1 \mathrm{H}), 7.41-7.31(\mathrm{~m}, 5 \mathrm{H}), 6.39-6.32(\mathrm{~m}$, $1 \mathrm{H}), 5.92-5.85(\mathrm{~m}, 1 \mathrm{H}), 5.16(\mathrm{~s}, 2 \mathrm{H}), 4.89(\mathrm{~s}, 1 \mathrm{H}), 4.85(\mathrm{~s}, 1 \mathrm{H}), 4.23-4.12(\mathrm{~m}, 2 \mathrm{H})$, $3.69-3.55(\mathrm{~m}, 2 \mathrm{H}), 3.52-3.39(\mathrm{~m}, 2 \mathrm{H}), 2.16-1.96(\mathrm{~m}, 2 \mathrm{H}), 1.74-1.67(\mathrm{~m}, 2 \mathrm{H})$, $1.66-1.58(\mathrm{~m}, 2 \mathrm{H}), 1.48-1.41(\mathrm{~m}, 2 \mathrm{H}), 0.98(\mathrm{t}, J=7.3 \mathrm{~Hz}, 3 \mathrm{H}) ;{ }^{13} \mathrm{C}$ NMR $(101$ $\left.\mathrm{MHz}, \mathrm{CDCl}_{3}\right) \delta 164.4,164.2,155.3,149.5,136.9,132.8,132.7,132.3,131.5,128.8$, 128.6, 128.1, 128.0, 127.4, 124.2, 123.0, 122.6, 122.0, 118.4, 86.2, 72.2, 67.3, 61.2, 42.3, 41.7, 40.3, 30.3, 20.4, 13.9; HRMS (ESI) m/z: $[\mathrm{M}+\mathrm{H}]^{+}$Calcd for $\mathrm{C}_{33} \mathrm{H}_{34} \mathrm{~N}_{3} \mathrm{O}_{5}$ 552.2493; Found 552.2512. 


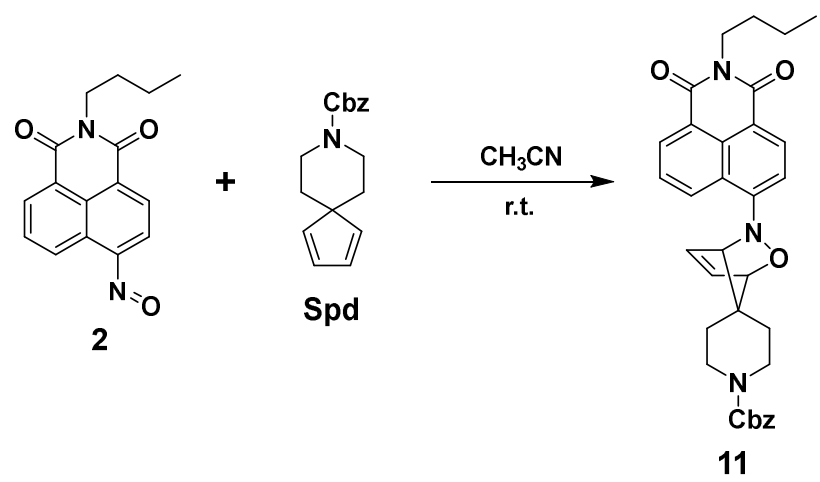

Synthesis of benzyl 3-(2-butyl-1,3-dioxo-2,3-dihydro-1H-benzo[de]isoquinolin-6yl)-2-oxa-3-azaspiro[bicyclo[2.2.1] hept[5]ene-7,4'-piperidine]-1'-carboxylate (11)

To a solution of $2(35 \mathrm{mg}, 0.124 \mathrm{mmol})$ in $\mathrm{CH}_{3} \mathrm{CN}(3 \mathrm{~mL})$ was added benzyl 8-azaspiro[4.5]deca-1,3-diene-8-carboxylate ${ }^{3}$ (Spd, $67 \mathrm{mg}, 0.25 \mathrm{mmol}$ ). The reaction mixture was stirred at r.t. for $5 \mathrm{~min}$, then concentrated. The residue was purified by silica gel flash column chromatography $(\mathrm{PE} / \mathrm{EtOAc}=4: 1)$ to afford the titled compound as yellow solid (64 mg, 94\% yield). m.p.: 185-188 ${ }^{\circ} \mathrm{C} ;{ }^{1} \mathrm{H}$ NMR (400 MHz, $\left.\mathrm{CDCl}_{3}\right) \delta 8.60(\mathrm{~d}, J=7.3 \mathrm{~Hz}, 1 \mathrm{H}), 8.45(\mathrm{~d}, J=8.2 \mathrm{~Hz}, 1 \mathrm{H}), 8.38(\mathrm{~d}, J=8.1 \mathrm{~Hz}, 1 \mathrm{H})$, $7.78-7.70(\mathrm{~m}, 1 \mathrm{H}), 7.46-7.32(\mathrm{~m}, 5 \mathrm{H}), 7.23(\mathrm{~d}, J=8.1 \mathrm{~Hz}, 1 \mathrm{H}), 6.56-6.50(\mathrm{~m}$, $1 \mathrm{H}), 5.59-5.52(\mathrm{~m}, 1 \mathrm{H}), 5.17(\mathrm{~s}, 2 \mathrm{H}), 4.93(\mathrm{~s}, 1 \mathrm{H}), 4.83(\mathrm{~s}, 1 \mathrm{H}), 4.22-4.10(\mathrm{~m}, 2 \mathrm{H})$, $3.74-3.67(\mathrm{~m}, 2 \mathrm{H}), 3.49-3.40(\mathrm{~m}, 2 \mathrm{H}), 2.31-2.09(\mathrm{~m}, 2 \mathrm{H}), 1.74-1.66(\mathrm{~m}, 2 \mathrm{H})$, $1.65-1.55(\mathrm{~m}, 2 \mathrm{H}), 1.47-1.40(\mathrm{~m}, 2 \mathrm{H}), 0.97(\mathrm{t}, J=7.3 \mathrm{~Hz}, 3 \mathrm{H}) ;{ }^{13} \mathrm{C}$ NMR $(101$ $\left.\mathrm{MHz}, \mathrm{CDCl}_{3}\right) \delta 164.4,164.0,155.4,151.9,136.8,131.8,131.7,131.0,130.5,129.1$, 129.0, 128.6, 128.2, 128.0, 126.0, 123.6, 123.1, 117.7, 116.4, 86.2, 75.0, 67.3, 61.4, 42.2, 41.9, 40.2, 30.3, 20.5, 13.9; HRMS (ESI) m/z: $[\mathrm{M}+\mathrm{H}]^{+}$Calcd for $\mathrm{C}_{33} \mathrm{H}_{34} \mathrm{~N}_{3} \mathrm{O}_{5}$ 552.2493; Found 552.2490.

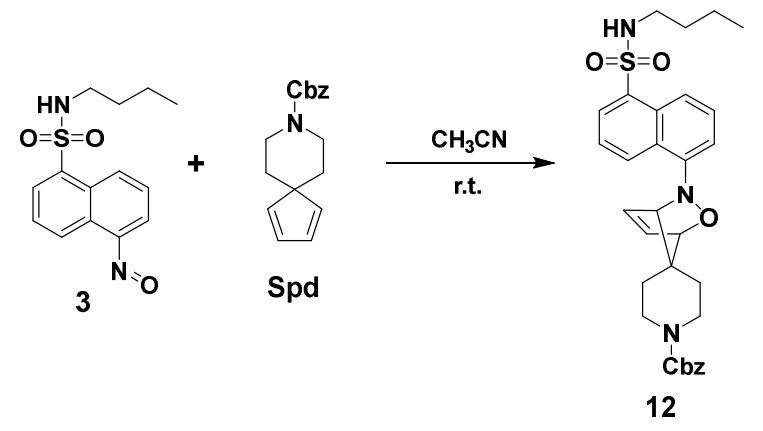

Synthesis of benzyl 3-(5-( $N$-butylsulfamoyl)naphthalen-1-yl)-2-oxa-3-azaspiro [bicyclo[2.2.1]hept[5]ene-7,4'-piperidine]-1'-carboxylate (12)

To a solution of $3(25 \mathrm{mg}, 0.086 \mathrm{mmol})$ in $\mathrm{CH}_{3} \mathrm{CN}(2 \mathrm{~mL})$ was added benzyl 8-azaspiro[4.5]deca-1,3-diene-8-carboxylate ${ }^{3}$ (Spd, $46 \mathrm{mg}, 0.17 \mathrm{mmol}$ ). The reaction mixture was stirred at r.t. for $5 \mathrm{~min}$, then concentrated. The residue was purified by silica gel flash column chromatography $(\mathrm{PE} / \mathrm{EtOAc}=2: 1)$ to afford the titled compound as yellow solid (44 mg, 92\% yield). m.p.: $155-157{ }^{\circ} \mathrm{C} ;{ }^{1} \mathrm{H}$ NMR (400 MHz, $\left.\mathrm{CDCl}_{3}\right) \delta 8.48(\mathrm{~d}, J=8.5 \mathrm{~Hz}, 1 \mathrm{H}), 8.27(\mathrm{t}, J=8.6 \mathrm{~Hz}, 2 \mathrm{H}), 7.54(\mathrm{t}, J=7.9 \mathrm{~Hz}, 1 \mathrm{H})$, 
$7.44(\mathrm{t}, J=8.1 \mathrm{~Hz}, 1 \mathrm{H}), 7.40-7.31(\mathrm{~m}, 5 \mathrm{H}), 7.12(\mathrm{~d}, J=7.6 \mathrm{~Hz}, 1 \mathrm{H}), 6.57-6.52(\mathrm{~m}$, $1 \mathrm{H}), 5.53-5.47(\mathrm{~m}, 1 \mathrm{H}), 5.17(\mathrm{~s}, 2 \mathrm{H}), 4.85(\mathrm{~s}, 1 \mathrm{H}), 4.63(\mathrm{~s}, 1 \mathrm{H}), 4.53(\mathrm{t}, J=6.0 \mathrm{~Hz}$, $1 \mathrm{H}), 3.70(\mathrm{t}, J=5.7 \mathrm{~Hz}, 2 \mathrm{H}), 3.44(\mathrm{t}, J=5.1 \mathrm{~Hz}, 2 \mathrm{H}), 2.99-2.89(\mathrm{~m}, 1 \mathrm{H}), 2.88-$ $2.78(\mathrm{~m}, 1 \mathrm{H}), 2.37-2.12(\mathrm{~m}, 2 \mathrm{H}), 1.37-1.30(\mathrm{~m}, 3 \mathrm{H}), 1.23-1.12(\mathrm{~m}, 3 \mathrm{H}), 0.74(\mathrm{t}, J$ $=7.3 \mathrm{~Hz}, 3 \mathrm{H}) ;{ }^{13} \mathrm{C}$ NMR $\left(101 \mathrm{MHz}, \mathrm{CDCl}_{3}\right) \delta 155.5,145.6,136.7,134.9,131.8$, 131.0, 130.6, 129.6, 128.8, 128.6, 128.2, 128.1, 127.8, 127.2, 123.6, 119.8, 117.3, 86.0, 73.6, 67.3, 61.3, 43.1, 42.3, 42.0, 31.6, 19.6, 13.5; HRMS (ESI) m/z: $[\mathrm{M}+\mathrm{H}]^{+}$ Calcd for $\mathrm{C}_{31} \mathrm{H}_{36} \mathrm{~N}_{3} \mathrm{O}_{5} \mathrm{~S} 562.2370$; Found 562.2372.

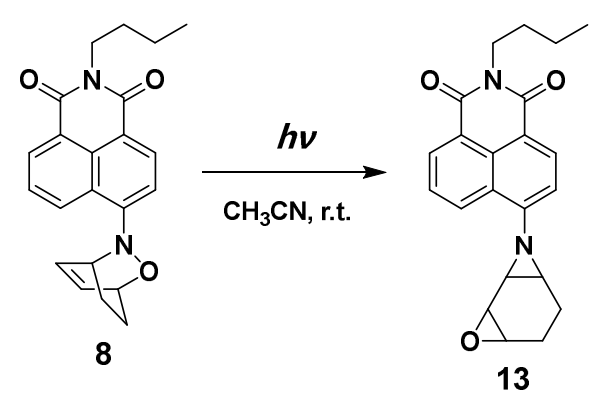

Synthesis of 2-butyl-6-(3-oxa-8-azatricyclo[5.1.0.0 $\left.{ }^{2,4}\right]$ octan-8-yl)-1H-benzo[de] isoquinoline-1,3(2H)-dione (13)

The solution of $8(30 \mathrm{mg}, 0.083 \mathrm{mmol})$ in $\mathrm{CH}_{3} \mathrm{CN}(10 \mathrm{~mL})$ was stirred at r.t and irradiated by UV light (365 $\mathrm{nm}$ wavelength) for $2 \mathrm{~h}$, then concentrated. The residue was purified by silica gel flash column chromatography (PE/EtOAc $=3: 1)$ to afford the titled compound as green solid (9 mg, 30\% yield). m.p.: 237-239 ${ }^{\circ} \mathrm{C} ;{ }^{1} \mathrm{H}$ NMR $\left(600 \mathrm{MHz}, \mathrm{CD}_{3} \mathrm{CN}\right) \delta 8.59(\mathrm{~d}, J=8.4 \mathrm{~Hz}, 1 \mathrm{H}), 8.52(\mathrm{~d}, J=7.2 \mathrm{~Hz}, 1 \mathrm{H}), 8.38(\mathrm{~d}, J=$ $7.9 \mathrm{~Hz}, 1 \mathrm{H}), 7.80(\mathrm{t}, J=7.8 \mathrm{~Hz}, 1 \mathrm{H}), 7.22$ (d, $J=7.9 \mathrm{~Hz}, 1 \mathrm{H}), 4.12-4.04(\mathrm{~m}, 2 \mathrm{H})$, $3.64(\mathrm{t}, J=3.7 \mathrm{~Hz}, 1 \mathrm{H}), 3.20-3.17(\mathrm{~m}, 1 \mathrm{H}), 2.89-2.85(\mathrm{~m}, 1 \mathrm{H}), 2.62-2.58(\mathrm{~m}, 1 \mathrm{H})$, $2.13-2.00(\mathrm{~m}, 2 \mathrm{H}), 1.95-1.88(\mathrm{~m}, 2 \mathrm{H}), 1.69-1.62(\mathrm{~m}, 2 \mathrm{H}), 1.45-1.37(\mathrm{~m}, 2 \mathrm{H})$, $0.97(\mathrm{t}, J=7.3 \mathrm{~Hz}, 3 \mathrm{H}) ;{ }^{13} \mathrm{C} \mathrm{NMR}\left(151 \mathrm{MHz}, \mathrm{CD}_{3} \mathrm{CN}\right) \delta 164.1,163.6,156.4,132.0$, 130.7, 129.2, 129.0, 126.2, 126.0, 123.2, 116.6, 115.2, 48.5, 47.1, 39.6, 36.9, 35.4, 30.0, 20.4, 20.1, 18.9, 13.2; HRMS (ESI) m/z: $[\mathrm{M}+\mathrm{H}]^{+}$Calcd for $\mathrm{C}_{22} \mathrm{H}_{23} \mathrm{~N}_{2} \mathrm{O}_{3}$ 363.1703; Found 363.1696.

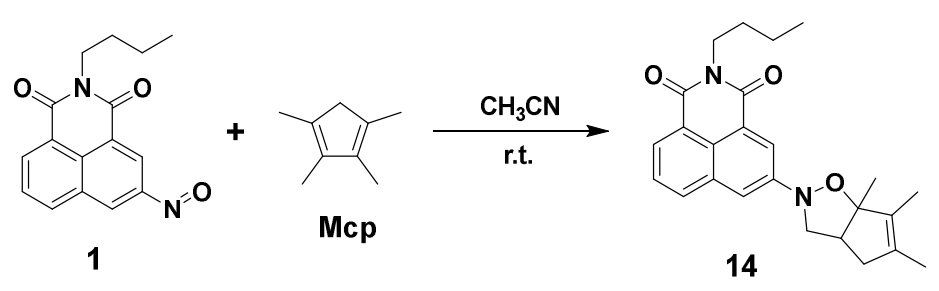

Synthesis of 2-butyl-5-(5,6,6a-trimethyl-3,3a,4,6a-tetrahydro-2H-cyclopenta[d] isoxazol-2-yl)-1H-benzo[de]isoquinoline-1,3(2H)-dione (14)

To a solution of $\mathbf{1}(80 \mathrm{mg}, 0.28 \mathrm{mmol})$ in $\mathrm{CH}_{3} \mathrm{CN}(7 \mathrm{~mL})$ was added 1,2,3,4-tetramethylcyclopenta-1,3-diene (Mcp, $69 \mathrm{mg}, 0.57 \mathrm{mmol}$ ). The reaction mixture was stirred at r.t. for $5 \mathrm{~min}$, then concentrated. The residue was purified by silica gel flash column chromatography $(\mathrm{PE} / \mathrm{EtOAc}=7: 1)$ to afford the titled 
compound as red solid (110 mg, 97\% yield). m.p.: 195-198 ${ }^{\circ} \mathrm{C} ;{ }^{1} \mathrm{H}$ NMR (600 MHz, $\left.\mathrm{CDCl}_{3}\right) \delta 8.36(\mathrm{~d}, J=6.3 \mathrm{~Hz}, 1 \mathrm{H}), 8.24(\mathrm{~s}, 1 \mathrm{H}), 8.01(\mathrm{~d}, J=7.9 \mathrm{~Hz}, 1 \mathrm{H}), 7.67(\mathrm{~s}, 1 \mathrm{H})$, $7.65-7.59(\mathrm{~m}, 1 \mathrm{H}), 4.20-4.12(\mathrm{~m}, 2 \mathrm{H}), 3.70-3.57(\mathrm{~m}, 2 \mathrm{H}), 2.86-2.80(\mathrm{~m}, 1 \mathrm{H})$, $2.68-2.58(\mathrm{~m}, 1 \mathrm{H}), 2.23-2.11(\mathrm{~m}, 1 \mathrm{H}), 1.72(\mathrm{~s}, 3 \mathrm{H}), 1.71-1.67(\mathrm{~m}, 2 \mathrm{H}), 1.52(\mathrm{~s}$, $3 \mathrm{H}), 1.50(\mathrm{~s}, 3 \mathrm{H}), 1.47-1.40(\mathrm{~m}, 2 \mathrm{H}), 0.97(\mathrm{t}, J=7.4 \mathrm{~Hz}, 3 \mathrm{H}) ;{ }^{13} \mathrm{C} \mathrm{NMR}(151 \mathrm{MHz}$, $\left.\mathrm{CDCl}_{3}\right) \delta 160.4,160.3,145.7,130.6,128.7,128.7,128.6,124.3,123.2,119.7,118.8$, $118.5,117.5,111.3,94.1,57.3,44.4,38.5,36.2,26.3,18.1,16.4,10.3,9.9,5.7$; HRMS (ESI) m/z: $[\mathrm{M}+\mathrm{H}]^{+}$Calcd for $\mathrm{C}_{25} \mathrm{H}_{29} \mathrm{~N}_{2} \mathrm{O}_{3} 405.2173$; Found 405.2162.

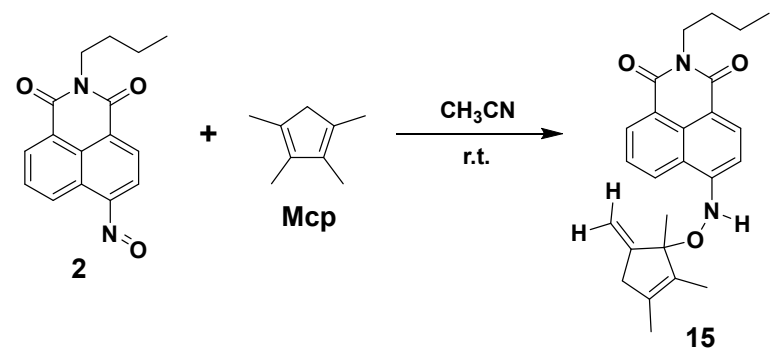

Synthesis of 2-butyl-6-(hydroxy(1,3,4-trimethyl-2-methylenecyclopent-3-en-1-yl) amino)-1H-benzo[de]isoquinoline-1,3(2H)-dione (15)

To a solution of $2(35 \mathrm{mg}, 0.124 \mathrm{mmol})$ in $\mathrm{CH}_{3} \mathrm{CN}(3 \mathrm{~mL})$ was added 1,2,3,4-tetramethylcyclopenta-1,3-diene (Mcp, $30 \mathrm{mg}, 0.25 \mathrm{mmol}$ ). The reaction mixture was stirred at r.t. for $5 \mathrm{~min}$, then concentrated. The residue was purified by silica gel flash column chromatography $(\mathrm{PE} /$ EtOAc $=5: 1)$ to afford the titled compound as yellow oil (47 mg, 93\% yield). ${ }^{1} \mathrm{H}$ NMR $\left(600 \mathrm{MHz}, \mathrm{CD}_{3} \mathrm{CN}\right) \delta 8.49$ (s, $1 \mathrm{H}), 8.38(\mathrm{~d}, J=7.2 \mathrm{~Hz}, 1 \mathrm{H}), 8.30(\mathrm{~d}, J=8.2 \mathrm{~Hz}, 1 \mathrm{H}), 8.09(\mathrm{~d}, J=8.4 \mathrm{~Hz}, 1 \mathrm{H}), 7.58(\mathrm{t}$, $J=7.8 \mathrm{~Hz}, 1 \mathrm{H}), 7.24(\mathrm{~d}, J=8.2 \mathrm{~Hz}, 1 \mathrm{H}), 5.02(\mathrm{~s}, 1 \mathrm{H}), 4.89(\mathrm{~s}, 1 \mathrm{H}), 4.05-3.96(\mathrm{~m}$, 2H), $2.73(\mathrm{~d}, J=17.5 \mathrm{~Hz}, 1 \mathrm{H}), 2.38(\mathrm{~d}, J=17.6 \mathrm{~Hz}, 1 \mathrm{H}), 1.77$ (s, 3H), 1.70 (s, 3H), $1.63-1.56(\mathrm{~m}, 2 \mathrm{H}), 1.51(\mathrm{~s}, 3 \mathrm{H}), 1.39-1.33(\mathrm{~m}, 2 \mathrm{H}), 0.93(\mathrm{t}, J=7.4 \mathrm{~Hz}, 3 \mathrm{H}) ;{ }^{13} \mathrm{C}$ NMR (151 MHz, CD $\left.{ }_{3} \mathrm{CN}\right) \delta 165.0,164.5,158.9,152.1,140.7,133.8,132.4,131.4$, 129.6, 128.1, 126.2, 123.7, 120.1, 114.7, 108.8, 102.0, 87.9, 48.3, 40.4, 31.0, 24.9, 21.1, 14.7, 14.2, 10.2; HRMS (ESI) m/z: $[\mathrm{M}+\mathrm{H}]^{+}$Calcd for $\mathrm{C}_{25} \mathrm{H}_{29} \mathrm{~N}_{2} \mathrm{O}_{3} 405.2173$; Found 405.2164 .

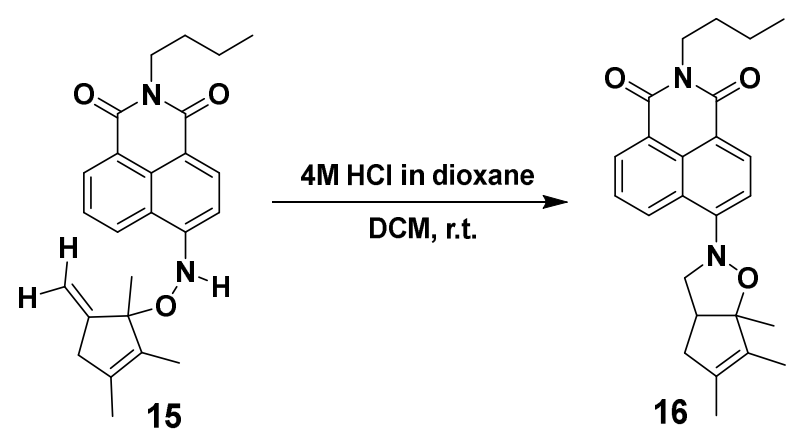

Synthesis of 2-butyl-6-(5,6,6a-trimethyl-3,3a,4,6a-tetrahydro-2 $H$-cyclopenta[d] isoxazol-2-yl)-1H-benzo[de] isoquinoline-1,3(2H)-dione (16) 
To a solution of 15 (30 mg, $0.074 \mathrm{mmol})$ in DCM (3 mL) was added $4 \mathrm{M} \mathrm{HCl}$ in dioxane $(1 \mathrm{~mL})$. The reaction mixture was stirred at r.t. for $10 \mathrm{~min}$, then concentrated. The residue was purified by silica gel flash column chromatography (PE/EtOAc $=5: 1)$ to afford the titled compound as yellow oil (27 mg, 90\% yield). ${ }^{1} \mathrm{H}$ NMR (400 MHz, $\left.\mathrm{CDCl}_{3}\right) \delta 8.54(\mathrm{~d}, J=8.1 \mathrm{~Hz}, 2 \mathrm{H}), 8.42(\mathrm{~d}, J=8.2 \mathrm{~Hz}, 1 \mathrm{H}), 7.65-7.58(\mathrm{~m}, 1 \mathrm{H}), 7.33$ $(\mathrm{d}, J=8.2 \mathrm{~Hz}, 1 \mathrm{H}), 4.19-4.10(\mathrm{~m}, 2 \mathrm{H}), 3.70(\mathrm{dd}, J=9.7,7.3 \mathrm{~Hz}, 1 \mathrm{H}), 3.59(\mathrm{dd}, J=$ 9.7, $2.9 \mathrm{~Hz}, 1 \mathrm{H}), 2.90-2.80(\mathrm{~m}, 1 \mathrm{H}), 2.70-2.58(\mathrm{~m}, 1 \mathrm{H}), 2.17(\mathrm{~d}, J=16.7 \mathrm{~Hz}, 1 \mathrm{H})$, $1.74(\mathrm{~s}, 3 \mathrm{H}), 1.72-1.65(\mathrm{~m}, 2 \mathrm{H}), 1.63(\mathrm{~s}, 3 \mathrm{H}), 1.51(\mathrm{~s}, 3 \mathrm{H}), 1.48-1.37$ (m, 2H), 0.95 $(\mathrm{t}, J=7.3 \mathrm{~Hz}, 3 \mathrm{H}) ;{ }^{13} \mathrm{C} \mathrm{NMR}\left(101 \mathrm{MHz}, \mathrm{CDCl}_{3}\right) \delta 164.5,164.0,152.4,135.2,132.4$, 132.3, 131.3, 131.1, 129.4, 125.2, 124.0, 122.6, 116.8, 111.9, 98.4, 62.6, 48.4, 42.5, 40.1, 30.3, 22.3, 20.4, 14.4, 13.9, 9.9; HRMS (ESI) m/z: $[\mathrm{M}+\mathrm{H}]^{+}$Calcd for $\mathrm{C}_{25} \mathrm{H}_{29} \mathrm{~N}_{2} \mathrm{O}_{3} 405.2173$; Found 405.2166.

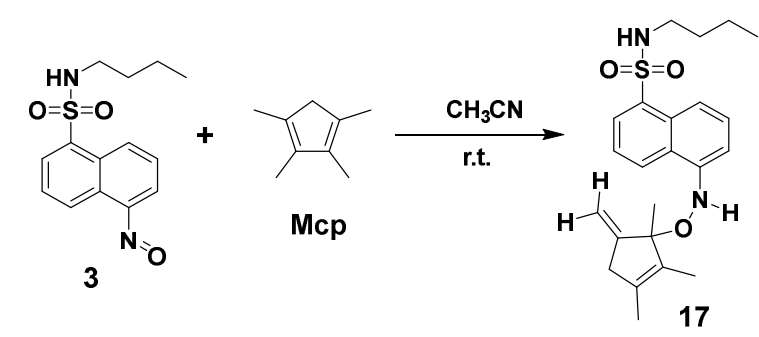

Synthesis of $N$-butyl-5-(hydroxy(1,3,4-trimethyl-2-methylenecyclopent-3-en-1-yl) amino)naphthalene-1-sulfonamide (17)

To a solution of $3(40 \mathrm{mg}, 0.137 \mathrm{mmol})$ in $\mathrm{CH}_{3} \mathrm{CN}(3 \mathrm{~mL})$ was added 1,2,3,4-tetramethylcyclopenta-1,3-diene (Mcp, $33 \mathrm{mg}, 0.27 \mathrm{mmol}$ ). The reaction mixture was stirred at r.t. for $5 \mathrm{~min}$, then concentrated. The residue was purified by silica gel flash column chromatography $(\mathrm{PE} / \mathrm{EtOAc}=6: 1)$ to afford the titled compound as yellow oil (52 mg, 91\% yield). ${ }^{1} \mathrm{H} \mathrm{NMR}\left(600 \mathrm{MHz}, \mathrm{CD}_{3} \mathrm{CN}\right) \delta 8.16(\mathrm{~d}$, $J=7.3 \mathrm{~Hz}, 1 \mathrm{H}), 8.13(\mathrm{~d}, J=8.7 \mathrm{~Hz}, 1 \mathrm{H}), 8.06(\mathrm{~d}, J=8.5 \mathrm{~Hz}, 1 \mathrm{H}), 7.96(\mathrm{~s}, 1 \mathrm{H}), 7.57$ $(\mathrm{t}, J=8.1 \mathrm{~Hz}, 1 \mathrm{H}), 7.51(\mathrm{t}, J=8.0 \mathrm{~Hz}, 1 \mathrm{H}), 7.33(\mathrm{~d}, J=7.6 \mathrm{~Hz}, 1 \mathrm{H}), 5.75(\mathrm{t}, J=5.9$ $\mathrm{Hz}, 1 \mathrm{H}), 5.01(\mathrm{~s}, 1 \mathrm{H}), 4.88(\mathrm{~s}, 1 \mathrm{H}), 2.82-2.73(\mathrm{~m}, 3 \mathrm{H}), 2.35(\mathrm{~d}, J=17.3 \mathrm{~Hz}, 1 \mathrm{H})$, $1.76(\mathrm{~s}, 3 \mathrm{H}), 1.71(\mathrm{~s}, 3 \mathrm{H}), 1.49(\mathrm{~s}, 3 \mathrm{H}), 1.29-1.23(\mathrm{~m}, 2 \mathrm{H}), 1.18-1.09(\mathrm{~m}, 2 \mathrm{H}), 0.70$ $(\mathrm{t}, J=7.4 \mathrm{~Hz}, 3 \mathrm{H}) ;{ }^{13} \mathrm{C} \mathrm{NMR}\left(151 \mathrm{MHz}, \mathrm{CD}_{3} \mathrm{CN}\right) \delta 158.8,145.9,139.9,135.5,131.4$, 129.01, 128.4, 128.3, 126.7, 123.2, 123.2, 116.9, 109.5, 100.3, 86.1, 47.4, 42.6, 31.2, 24.5, 19.3, 13.8, 12.7, 9.2; HRMS (ESI) m/z: $[\mathrm{M}+\mathrm{H}]^{+}$Calcd for $\mathrm{C}_{23} \mathrm{H}_{31} \mathrm{~N}_{2} \mathrm{O}_{3} \mathrm{~S}$ 415.2050; Found 415.2039.

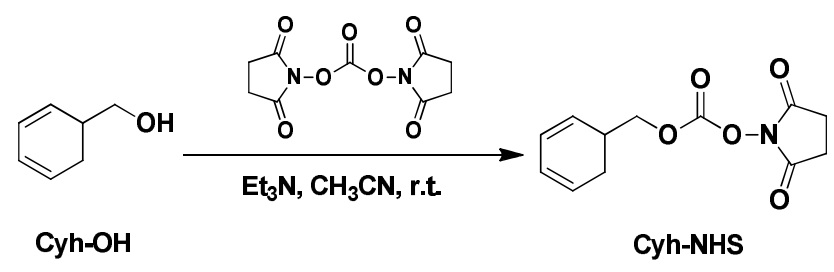

Synthesis of cyclohexa-2,4-dien-1-ylmethyl (2,5-dioxopyrrolidin-1-yl) carbonate (Cyh-NHS) 
To a solution of $\mathbf{C y h}-\mathbf{O H}^{7}$ (54 mg, $0.49 \mathrm{mmol}$ ) in $\mathrm{CH}_{3} \mathrm{CN}(5 \mathrm{~mL})$ was added $\mathrm{Et}_{3} \mathrm{~N}$ (198 mg, $1.96 \mathrm{mmol}$ ) and $N, N^{\prime}$-disuccinimidyl carbonate (251 mg, $0.98 \mathrm{mmol}$ ). The reaction mixture was stirred at r.t. for $12 \mathrm{~h}$, then concentrated. The residue was purified by silica gel flash column chromatography $(\mathrm{PE} / \mathrm{EtOAc}=4: 1)$ to afford the titled compound as colorless oil (96 mg, 78\% yield). ${ }^{1} \mathrm{H}$ NMR (400 $\left.\mathrm{MHz}, \mathrm{CDCl}_{3}\right) \delta$ $6.12-5.53(\mathrm{~m}, 4 \mathrm{H}), 4.27-4.19(\mathrm{~m}, 2 \mathrm{H}), 2.83(\mathrm{~s}, 4 \mathrm{H}), 2.40-2.05(\mathrm{~m}, 3 \mathrm{H}) ;{ }^{13} \mathrm{C} \mathrm{NMR}$ $\left(101 \mathrm{MHz}, \mathrm{CDCl}_{3}\right) \delta 168.8,151.7,127.1,126.6,125.1,124.3,72.0,32.1,27.6,25.5$; HRMS (ESI) m/z: $[\mathrm{M}+\mathrm{H}]^{+}$Calcd for $\mathrm{C}_{12} \mathrm{H}_{14} \mathrm{NO}_{5} 252.0851$; Found 252.0844.

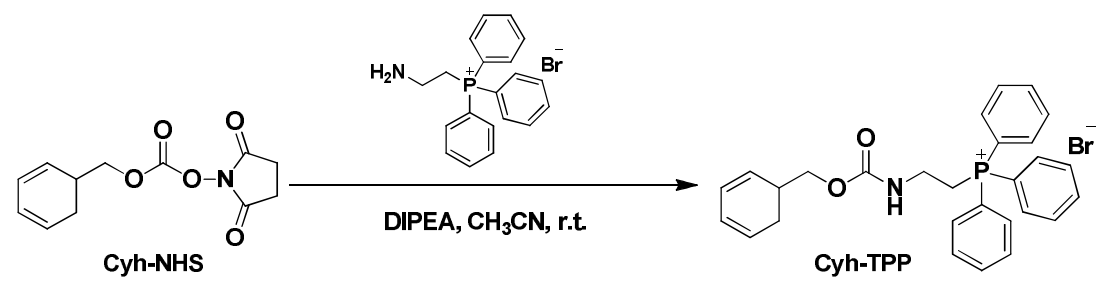

Synthesis of (2-(((cyclohexa-2,4-dien-1-ylmethoxy)carbonyl)amino)ethyl) triphenylphosphonium bromide (Cyh-TPP)

To a solution of Cyh-NHS (34 mg, $0.135 \mathrm{mmol})$ in $\mathrm{CH}_{3} \mathrm{CN}(3 \mathrm{~mL})$ was added DIPEA (44 mg, $0.34 \mathrm{mmol}$ ) and (2-aminoethyl)triphenylphosphonium bromide (58 $\mathrm{mg}, 0.15 \mathrm{mmol}$ ). The reaction mixture was stirred at r.t. for $4 \mathrm{~h}$, then concentrated. The residue was purified by silica gel flash column chromatography (DCM/MeOH = 10:1) to afford the titled compound as white solid (64 mg, 91\% yield). m.p.: 139-141 ${ }^{\circ} \mathrm{C} ;{ }^{1} \mathrm{H}$ NMR (400 MHz, CD $\left.3 \mathrm{OD}\right) \delta 7.91-7.73(\mathrm{~m}, 16 \mathrm{H}), 6.15-5.55(\mathrm{~m}, 4 \mathrm{H}), 4.03-$ $3.80(\mathrm{~m}, 2 \mathrm{H}), 3.75-3.63(\mathrm{~m}, 2 \mathrm{H}), 3.60-3.50(\mathrm{~m}, 2 \mathrm{H}), 2.24-1.80(\mathrm{~m}, 3 \mathrm{H}) ;{ }^{13} \mathrm{C}$ NMR (101 MHz, CD $\left.{ }_{3} \mathrm{OD}\right) \delta 157.4,135.0,133.5,133.4,130.2,130.1,126.5,125.1$, 118.6, 117.7, 69.0, 34.5, 33.2, 24.9, 24.0; HRMS (ESI) m/z: [M] Calcd for $\mathrm{C}_{28} \mathrm{H}_{29} \mathrm{NO}_{2} \mathrm{P}$ 442.1930; Found 442.1949.

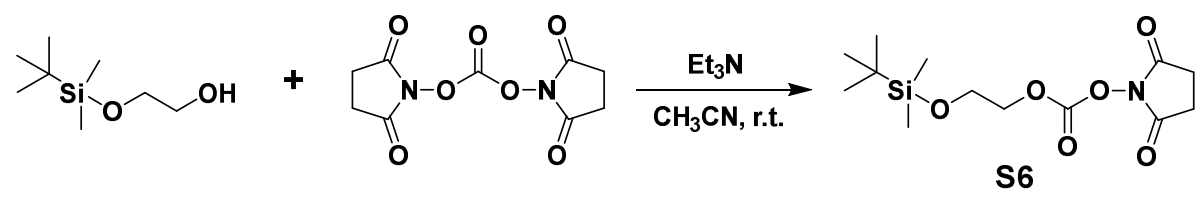

\section{Synthesis of 2-((tert-butyldimethylsilyl)oxy)ethyl (2,5-dioxopyrrolidin-1-yl)} carbonate (S6)

To a solution of 2-((tert-butyldimethylsilyl)oxy)ethanol (2 $\mathrm{g}, 11.3 \mathrm{mmol})$ in $\mathrm{CH}_{3} \mathrm{CN}(20 \mathrm{~mL})$ was added $\mathrm{Et}_{3} \mathrm{~N}(3.43 \mathrm{~g}, 33.9 \mathrm{mmol})$ and $N, N^{\prime}$-disuccinimidyl carbonate $(4.36 \mathrm{~g}, 17 \mathrm{mmol})$. The reaction mixture was stirred at r.t. for $2 \mathrm{~h}$, then concentrated. The residue was purified by silica gel flash column chromatography $(\mathrm{PE} / \mathrm{EtOAc}=3: 1)$ to afford the titled compound as white solid (3.2 $\mathrm{g}, 89 \%$ yield). m.p.: 98-99 ${ }^{\circ} \mathrm{C} ;{ }^{1} \mathrm{H}$ NMR $\left(400 \mathrm{MHz}, \mathrm{CDCl}_{3}\right) \delta 4.36-4.31(\mathrm{~m}, 2 \mathrm{H}), 3.88-3.83(\mathrm{~m}$, 2H), $2.79(\mathrm{~s}, 4 \mathrm{H}), 0.86(\mathrm{~s}, 9 \mathrm{H}), 0.06(\mathrm{~s}, 6 \mathrm{H}) ;{ }^{13} \mathrm{C} \mathrm{NMR}\left(101 \mathrm{MHz}, \mathrm{CDCl}_{3}\right) \delta 168.8$, 151.8, 72.2, 60.7, 25.9, 25.5, 18.3, 5.4; HRMS (ESI) m/z: $[\mathrm{M}+\mathrm{H}]^{+}$Calcd for $\mathrm{C}_{13} \mathrm{H}_{24} \mathrm{NO}_{6} \mathrm{Si} 318.1367$; Found 318.1379. 


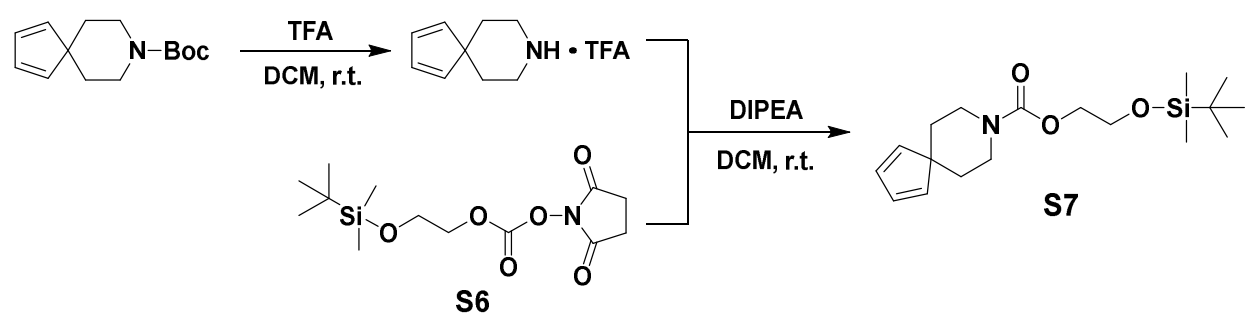

Synthesis of 2-((tert-butyldimethylsilyl)oxy)ethyl 8-azaspiro[4.5]deca-1,3-diene-8carboxylate (S7)

To a solution of tert-butyl 8-azaspiro[4.5]deca-1,3-diene-8-carboxylate ${ }^{6}$ (500 mg, $2.12 \mathrm{mmol})$ in DCM $(10 \mathrm{~mL})$ was added TFA $(2 \mathrm{~mL})$. The reaction mixture was stirred at r.t. for $1 \mathrm{~h}$, then concentrated. The residue was redissolved in DCM (20 mL), then DIPEA (822 mg, $6.4 \mathrm{mmol})$ and $\mathbf{S 6}(674 \mathrm{mg}, 2.12 \mathrm{mmol})$ were added. The reaction mixture was stirred at r.t. for $12 \mathrm{~h}$, then concentrated. The residue was purified by silica gel flash column chromatography $(\mathrm{PE} / \mathrm{EtOAc}=4: 1)$ to afford the titled compound as yellow oil (380 mg, 53\% yield). ${ }^{1} \mathrm{H}$ NMR (400 $\left.\mathrm{MHz}, \mathrm{CDCl}_{3}\right) \delta$ $6.50-6.46(\mathrm{~m}, 2 \mathrm{H}), 6.33-6.29(\mathrm{~m}, 2 \mathrm{H}), 4.20-4.15(\mathrm{~m}, 2 \mathrm{H}), 3.85-3.80(\mathrm{~m}, 2 \mathrm{H})$, $3.65-3.59(\mathrm{~m}, 4 \mathrm{H}), 1.59-1.50(\mathrm{~m}, 4 \mathrm{H}), 0.90(\mathrm{~s}, 9 \mathrm{H}), 0.07(\mathrm{~s}, 6 \mathrm{H}) ;{ }^{13} \mathrm{C}$ NMR $(101$ $\left.\mathrm{MHz}, \mathrm{CDCl}_{3}\right) \delta 155.6,142.5,129.7,66.7,61.8,55.4,43.4,30.9,25.9,18.4,5.2$; HRMS (ESI) m/z: [M + H] $]^{+}$Calcd for $\mathrm{C}_{18} \mathrm{H}_{32} \mathrm{NO}_{3} \mathrm{Si} 338.2146$; Found 338.2147.

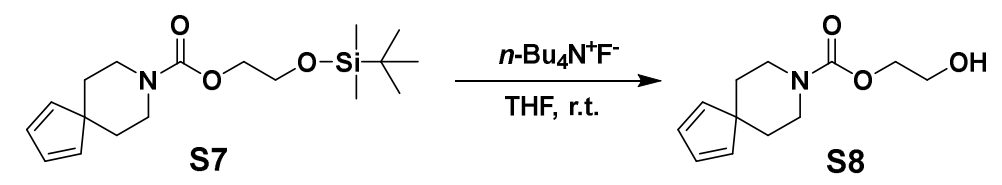

Synthesis of 2-hydroxyethyl 8-azaspiro[4.5]deca-1,3-diene-8-carboxylate (S8)

To a solution of $\mathbf{S} 7(340 \mathrm{mg}, 1 \mathrm{mmol})$ in THF $(6 \mathrm{~mL})$ was added $1 \mathrm{Mn}$-Bu $4 \mathrm{~N}^{+} \mathrm{F}^{-}$ $(2.5 \mathrm{~mL}, 2.5 \mathrm{mmol})$. The reaction mixture was stirred at r.t. for $1 \mathrm{~h}$, then concentrated. The residue was purified by silica gel flash column chromatography $(\mathrm{PE} / \mathrm{EtOAc}=1: 1)$ to afford the titled compound as yellow oil (212 mg, 95\% yield). ${ }^{1} \mathrm{H}$ NMR (400 MHz, $\left.\mathrm{CDCl}_{3}\right) \delta 6.45-6.39(\mathrm{~m}, 2 \mathrm{H}), 6.28-6.22(\mathrm{~m}, 2 \mathrm{H}), 4.21-4.16(\mathrm{~m}, 2 \mathrm{H}), 3.78-3.72$ $(\mathrm{m}, 2 \mathrm{H}), 3.60-3.53(\mathrm{~m}, 4 \mathrm{H}), 3.33$ (brs, $1 \mathrm{H}), 1.54-1.45(\mathrm{~m}, 4 \mathrm{H}) ;{ }^{13} \mathrm{C}$ NMR $(101$ $\left.\mathrm{MHz}, \mathrm{CDCl}_{3}\right) \delta 156.1,142.4,129.8,67.4,61.8,55.2,43.5,30.8 ;$ HRMS (ESI) m/z: $[\mathrm{M}+\mathrm{H}]^{+}$Calcd for $\mathrm{C}_{12} \mathrm{H}_{18} \mathrm{NO}_{3} 224.1281$; Found 224.1276.

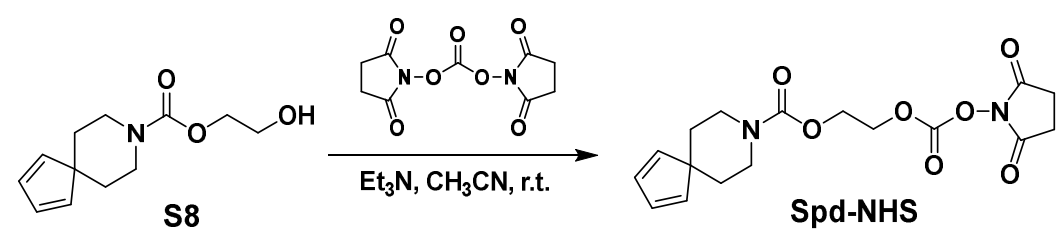

Synthesis of 2-(((2,5-dioxopyrrolidin-1-yl)oxy)carbonyl)oxy)ethyl 8-azaspiro[4.5] deca-1,3-diene-8-carboxylate (Spd-NHS) 
To a solution of $\mathbf{S 8}(180 \mathrm{mg}, 0.81 \mathrm{mmol})$ in $\mathrm{CH}_{3} \mathrm{CN}(5 \mathrm{~mL})$ was added $\mathrm{Et}_{3} \mathrm{~N}$ (246 $\mathrm{mg}, 2.43 \mathrm{mmol}$ ) and $N, N^{\prime}$-disuccinimidyl carbonate (228 $\mathrm{mg}, 0.89 \mathrm{mmol}$ ). The reaction mixture was stirred at r.t. for $12 \mathrm{~h}$, then concentrated. The residue was purified by silica gel flash column chromatography $(\mathrm{DCM} / \mathrm{EtOAc}=2: 1)$ to afford the titled compound as colorless solid (285 mg, 97\% yield). m.p.: 103-105 ${ }^{\circ} \mathrm{C} ;{ }^{1} \mathrm{H}$ NMR $\left(600 \mathrm{MHz}, \mathrm{CDCl}_{3}\right) \delta 6.49(\mathrm{~d}, J=5.9 \mathrm{~Hz}, 2 \mathrm{H}), 6.31(\mathrm{~d}, J=5.9 \mathrm{~Hz}, 2 \mathrm{H}), 4.55(\mathrm{t}, J=5.7$ $\mathrm{Hz}, 2 \mathrm{H}), 4.38$ (t, $J=5.7 \mathrm{~Hz}, 2 \mathrm{H}), 3.61(\mathrm{t}, J=5.4 \mathrm{~Hz}, 4 \mathrm{H}), 2.83(\mathrm{~s}, 4 \mathrm{H}), 1.55$ (t, $J=5.7$ $\mathrm{Hz}, 4 \mathrm{H}) ;{ }^{13} \mathrm{C} \mathrm{NMR}\left(151 \mathrm{MHz}, \mathrm{CDCl}_{3}\right) \delta 168.5,154.7,151.6,142.4,129.6,69.3,62.4$, 55.2, 43.4, 30.8, 25.4; HRMS (ESI) m/z: $[\mathrm{M}+\mathrm{H}]^{+}$Calcd for $\mathrm{C}_{17} \mathrm{H}_{21} \mathrm{~N}_{2} \mathrm{O}_{7} 365.1343$; Found 365.1347.

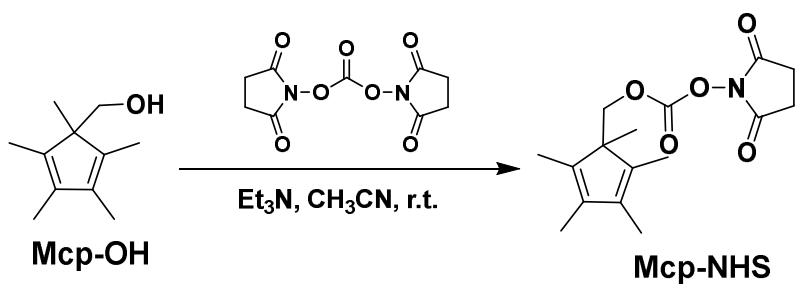

Synthesis of 2,5-dioxopyrrolidin-1-yl ((1,2,3,4,5-pentamethylcyclopenta-2,4-dien -1-yl)methyl) carbonate (Mcp-NHS)

To a solution of Mcp-OH ${ }^{8}$ (70 mg, $\left.0.42 \mathrm{mmol}\right)$ in $\mathrm{CH}_{3} \mathrm{CN}(5 \mathrm{~mL})$ was added $\mathrm{Et}_{3} \mathrm{~N}$ (340 mg, $3.36 \mathrm{mmol}$ ) and $N, N^{\prime}$-disuccinimidyl carbonate $(431 \mathrm{mg}, 1.68 \mathrm{mmol}$ ). The reaction mixture was stirred at r.t. for $12 \mathrm{~h}$, then concentrated. The residue was purified by silica gel flash column chromatography $(\mathrm{PE} / \mathrm{EtOAc}=5: 1)$ to afford the titled compound as white solid (123 mg, 95\% yield). m.p.: 137-139 ${ }^{\circ} \mathrm{C} ;{ }^{1} \mathrm{H}$ NMR (400 $\left.\mathrm{MHz}, \mathrm{CDCl}_{3}\right) \delta 4.15(\mathrm{~s}, 2 \mathrm{H}), 2.80(\mathrm{~s}, 4 \mathrm{H}), 1.76(\mathrm{~d}, J=3.6 \mathrm{~Hz}, 12 \mathrm{H}), 1.00(\mathrm{~s}, 3 \mathrm{H}) ;{ }^{13} \mathrm{C}$ NMR $\left(151 \mathrm{MHz}, \mathrm{CDCl}_{3}\right) \delta 168.5,151.4,137.5,136.0,74.8,55.4,25.4,16.5,11.1$, 10.1; HRMS (ESI) m/z: [M+ H $]^{+}$Calcd for $\mathrm{C}_{16} \mathrm{H}_{22} \mathrm{NO}_{5} 308.1493$; Found 308.1486.

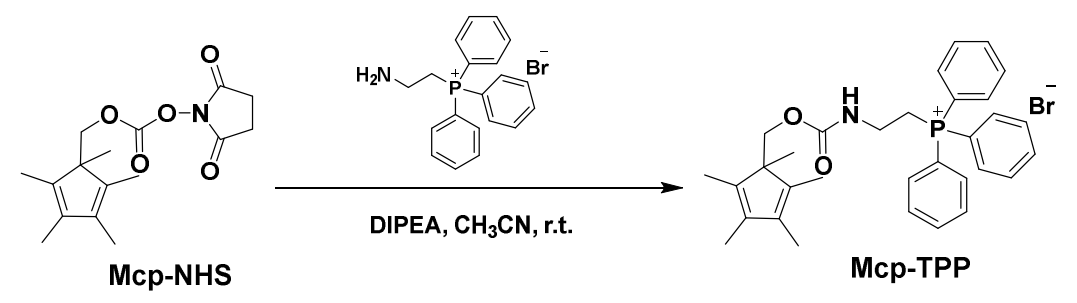

Synthesis of (2-((((1,2,3,4,5-pentamethylcyclopenta-2,4-dien-1-yl)methoxy) carbonyl)amino)ethyl)triphenylphosphonium bromide (Mcp-TPP)

To a solution of Mcp-NHS (34 mg, $0.11 \mathrm{mmol}$ ) in $\mathrm{CH}_{3} \mathrm{CN}(2 \mathrm{~mL})$ was added DIPEA (36 mg, $0.28 \mathrm{mmol}$ ) and (2-aminoethyl) triphenylphosphonium bromide (47 $\mathrm{mg}, 0.12 \mathrm{mmol}$ ). The reaction mixture was stirred at r.t. for $4 \mathrm{~h}$, then concentrated. The residue was purified by silica gel flash column chromatography (DCM/MeOH = 10:1) to afford the titled compound as yellow solid (55 mg, 87\% yield). m.p.: 132-135 ${ }^{\circ} \mathrm{C} ;{ }^{1} \mathrm{H}$ NMR (400 MHz, CD $\left.3 \mathrm{OD}\right) \delta 7.92-7.77(\mathrm{~m}, 16 \mathrm{H}), 3.85(\mathrm{~s}, 2 \mathrm{H}), 3.69-3.59$ (m, 
2H), $3.54-3.45(\mathrm{~m}, 2 \mathrm{H}), 1.75(\mathrm{~d}, J=3.7 \mathrm{~Hz}, 12 \mathrm{H}), 0.93(\mathrm{~s}, 3 \mathrm{H}) ;{ }^{13} \mathrm{C}$ NMR $(101 \mathrm{MHz}$, $\left.\mathrm{CD}_{3} \mathrm{OD}\right) \delta 157.3,138.3,135.0,134.5,133.5,133.4,130.2,130.1,118.5,117.7,68.0$, 55.3, 34.5, 29.3, 16.2, 9.7, 8.9; HRMS (ESI) m/z: $[\mathrm{M}]^{+}$Calcd for $\mathrm{C}_{32} \mathrm{H}_{37} \mathrm{NO}_{2} \mathrm{P}$ 498.2556; Found 498.2546.

\section{X-ray single crystal diffraction}

Suitable crystals of compound $\mathbf{8}, \mathbf{1 3}, \mathbf{1 4}$ were obtained by slowly evaporating a mixture of chloroform and methanol solution $(1: 1, \mathrm{v} / \mathrm{v})$ at ambient temperature. A colorless crystal of 8,13 or 14 was mounted on a glass fiber at a random orientation. The data were collected at $100 \mathrm{~K}$ by a diffractometer Rigaku Oxford Diffraction Supernova Dual Source, $\mathrm{Cu}$ at Zero equipped with an AtlasS2 $\mathrm{CCD}$ using $\mathrm{Cu} \mathrm{K \alpha}$ radiation (1.54178 $\AA$ ), and processed using CrysAlisPro ${ }^{9}$. The structures were solved by direct methods using Olex 2 software ${ }^{10}$, and the nonhydrogen atoms were located from the trial structure and then refined anisotropically with SHELXL-2018 ${ }^{11}$ using a full-matrix least squares procedure based on $\mathrm{F}^{2}$. The weighted $R$ factor, $w R$ and goodness-of-fit $S$ values were obtained based on $\mathrm{F}^{2}$. The hydrogen atom positions were fixed geometrically at the calculated distances and allowed to ride on the parent atoms. Crystallographic data for the structure reported in this paper have been deposited at the Cambridge Crystallographic Data Center and allocated with the deposition numbers: CCDC 2054925 for compound 8, 2054928 for compound 13, and 2054926 for compound 14.

\section{Absorption and fluorescence spectra of probes}

The stock solution of each 1,8-naphthalimide or naphthalene-1-sulfonamide probe was prepared at $10 \mathrm{mM}$ in DMSO. A fresh work solution of corresponding probe was prepared by diluting the stock solution to $\mathrm{CH}_{3} \mathrm{CN}$ to make a final concentration of 10 $\mu \mathrm{M}$. The absorption and fluorescence spectra were recorded using a Tecan Spark ${ }^{\mathrm{TM}}$ $10 \mathrm{M}$ Multimode Microplate Reader. The absorption spectra were recorded from 300 $\mathrm{nm}$ to $550 \mathrm{~nm}$, and the fluorescence spectra were recorded from $410 \mathrm{~nm}$ to $720 \mathrm{~nm}$ with an excitation wavelength of $365 \mathrm{~nm}$. The data were subtracted from the background absorbance or fluorescence signal of $\mathrm{CH}_{3} \mathrm{CN}$ for the same time period. The fluorescence images of naphthylene probes in cuvettes were captured as soon as the excited UV lamp turned on.

For UV-irradiated probes, $200 \mu \mathrm{L}$ of $10 \mu \mathrm{M}$ 1,8-naphthalimide or naphthalene-1-sulfonamide probe in $\mathrm{CH}_{3} \mathrm{CN}$ was put into 96-well plate (polystyrene) and exposed to 365-nm UV light from a handheld UV lamp (UVP, 3UV-38, $8 \mathrm{~W}$, placed on the top of the 96 -well plate at a distance of $1 \mathrm{~cm}$ ) on ice for certain time, then the absorption and fluorescence spectra were recorded using the same method mentioned above.

For the in situ fluorescence measurement in the presence of nucleophilic amino acids, $1 \mu \mathrm{L}$ of $10 \mathrm{mM} 2$ in DMSO was added to $979 \mu \mathrm{L}$ PBS ( $\mathrm{pH}=7.4)$, then $10 \mu \mathrm{L}$ of $10 \mathrm{mM}$ diene and $10 \mu \mathrm{L}$ of $10 \mathrm{mM}$ amino acids (Ser, Glu, Lys, His, Arg, Met and Cys) 
in PBS ( $\mathrm{pH}=7.4$ ) were added to the solution, followed by incubation at r.t. for $30 \mathrm{~min}$. For the in situ fluorescence measurement in cell lysates, $1 \mu \mathrm{L}$ of $10 \mathrm{mM} 2$ in DMSO was added to $989 \mu \mathrm{L}$ HepG2 cell lysates in PBS ( $\mathrm{pH}=7.4$ ), then $10 \mu \mathrm{L}$ of $10 \mathrm{mM}$ diene in PBS ( $\mathrm{pH}=7.4)$ were added to the solution, followed by incubation at r.t. for $30 \mathrm{~min}$. Then $200 \mu \mathrm{L}$ of the mixture was put into 96-well plate (polystyrene) and exposed to 365-nm UV light from a handheld UV lamp (UVP, 3UV-38, $8 \mathrm{~W}$, placed on the top of the 96-well plate at a distance of $1 \mathrm{~cm}$ ) on ice for $30 \mathrm{~min}$, then the fluorescence spectra were recorded using the same method mentioned above.

\section{Reaction kinetics measurement}

The nitroso-diene reaction kinetics measurements were performed at room temperature in TECAN fluorescence plate reader equipped with automatic injection unit. Two $\mu \mathrm{L}$ of $10 \mathrm{mM} 2$ in $\mathrm{DMSO} / \mathrm{H}_{2} \mathrm{O}(4: 1, \mathrm{v} / \mathrm{v})$ was pipetted into each well containing $178 \mu \mathrm{L}$ DMSO/ $\mathrm{H}_{2} \mathrm{O}(4: 1, \mathrm{v} / \mathrm{v})$. To this solution was added $20 \mu \mathrm{L}$ of 10 $\mathrm{mM}$ Cyh, Spd or Mcp in DMSO/ $\mathrm{H}_{2} \mathrm{O}(4: 1, \mathrm{v} / \mathrm{v})$. The final concentration of 2 was 100 $\mu \mathrm{M}$, and the final concentration of Cyh, Spd or Mcp was $1 \mathrm{mM}$. The well plate was inserted into plate reader and the measurement was immediately started. For the reaction with Cyh or Spd, the absorbance intensities were collected at $414 \mathrm{~nm}$ for Cyh and $408 \mathrm{~nm}$ for Spd. For the reaction with Mcp, excitation was set to $365 \mathrm{~nm}$ and the fluorescence intensities were collected at $535 \mathrm{~nm}$. All experiments were performed in triplicate and average values of absorbance or fluorescence intensities were plotted as the function of time. The observed rate constants $\left(k_{o b s}\right)$ were calculated from the plots by fitting the curves with single exponential growth function. The second order rate constants $\left(k_{2}\right)$ were calculated using the following equation: $k_{2}=k_{o b s} /[$ diene].

\section{BSA protein labeling}

For preparation of BSA-Cyh/Spd/Mcp conjugates (diene-modified BSA protein), a solution of BSA ( $400 \mu \mathrm{L}, 20 \mathrm{mg} / \mathrm{mL})$ in PBS ( $\mathrm{pH}$ 7.4) was incubated with a solution of $\mathrm{Cyh} / \mathrm{Spd} / \mathrm{Mcp}-\mathrm{NHS}$ ester $(100 \mu \mathrm{L}, 25 \mathrm{mM})$ in DMSO overnight at room temperature. The excess of $\mathrm{Cyh} / \mathrm{Spd} / \mathrm{Mcp}-\mathrm{NHS}$ ester was removed by spin-filtration $(\mathrm{MWCO}=3 \mathrm{kDa})$. The obtained BSA-Cyh/Spd/Mcp conjugates was then redissolved in PBS ( $\mathrm{pH} 7.4$ ) to a final concentration of $2 \mathrm{mg} / \mathrm{mL}$.

For in-gel fluorogenic labeling, $1 \mu \mathrm{L}$ of $10 \mathrm{mM} 2$ in DMSO was added to $9 \mu \mathrm{L}$ $\mathrm{CH}_{3} \mathrm{CN}$, then $10 \mu \mathrm{L}$ of BSA-Cyh/Spd/Mcp conjugates $(2 \mathrm{mg} / \mathrm{mL}, 30 \mu \mathrm{M})$ in PBS (pH 7.4) was added to the solution. The mixture was incubated at r.t. for $1 \mathrm{~h}$. The solvent was removed by freeze drying and the resulting protein was redissolved in $20 \mu \mathrm{L}$ PBS (pH 7.4) and analysed by SDS-PAGE. The SDS-PAGE gel was exposed to 365-nm UV light from a handheld UV lamp (UVP, 3UV-38, $8 \mathrm{~W}$, placed on the top of the gel at a distance of $1 \mathrm{~cm}$ ) for $0,5,10 \mathrm{~min}$, and the fluorescence images were recorded. Finally, the gel was subjected to coomassie blue staining.

For time-dependent photoactivated fluorogenic labeling, $1 \mu \mathrm{L}$ of $10 \mathrm{mM} 2$ in DMSO was added to $9 \mu \mathrm{L} \mathrm{CH}_{3} \mathrm{CN}$, then $10 \mu \mathrm{L}$ of BSA-Cyh conjugates $(2 \mathrm{mg} / \mathrm{mL}$, 
$30 \mu \mathrm{M}$ ) in PBS ( $\mathrm{pH}$ 7.4) was added to the solution. The mixture was incubated at r.t. for $1 \mathrm{~h}$, then was put into 96-well plate (polystyrene) and exposed to 365-nm UV light from a handheld UV lamp (UVP, 3UV-38, $8 \mathrm{~W}$, placed on the top of the 96-well plate at a distance of $1 \mathrm{~cm}$ ) on ice for $0,0.5,1,2,5,10,15 \mathrm{~min}$. The mixture was analysed by SDS-PAGE and the fluorescence images were recorded. Finally, the gel was subjected to coomassie blue staining.

\section{In gel digestion and LC-MS/MS analysis}

The bands of interest were excised from gels and cut into cubes $(1 \times 1 \mathrm{~mm})$. The gel pieces were destained and dehydrated in acetonitrile and incubated in buffer I (10 $\mathrm{mM}$ DTT, $\left.100 \mathrm{mM} \mathrm{NH} 4 \mathrm{HCO}_{3}\right)$ at $56{ }^{\circ} \mathrm{C}$ for $30 \mathrm{~min}$ followed by buffer II $(55 \mathrm{mM}$ iodoacetamide, $100 \mathrm{mM} \mathrm{NH}_{4} \mathrm{HCO}_{3}$ ) at ambient temperature for $20 \mathrm{~min}$ before being dehydrated. The samples were in-gel digested with trypsin $(5 \mathrm{ng} / \mu 1$ trypsin in $50 \mathrm{mM}$ ammonium bicarbonate, $\mathrm{pH} 8.0$ ) at $37{ }^{\circ} \mathrm{C}$ overnight. The resulting peptides were extracted twice with extraction buffer (5\% formic acid/acetonitrile, 1:2) and then vacuum-centrifuged to dryness. For LC-MS/MS analysis, the extracted peptides were reconstituted in $0.2 \%$ formic acid, and separated on a $1 \mathrm{~m}$ long, $75 \mu \mathrm{m}$ I.D., $3 \mu \mathrm{m}$ particle size column under a flow rate of $250 \mu \mathrm{l} / \mathrm{min}$ with a 2 -hour gradient. The eluted peptides were analyzed by Orbitrap-Lumos mass spectrometer (Thermo) in CID-IT mode.

The raw data files were searched with target-decoy combined database using Thermo Proteome Discoverer 2.5 software. Searches were performed with a precursor mass tolerance of $10 \mathrm{ppm}$ and fragment mass tolerance of $0.6 \mathrm{Da}$. In addition to regular methionine oxidation $(\mathrm{O}, 15.9949 \mathrm{Da})$, a variable modification $\left(\mathrm{C}_{24} \mathrm{H}_{23} \mathrm{~N}_{2} \mathrm{O}_{5}\right.$, $+419.16069 \mathrm{Da}$ ) on each lysine were defined in the search to account for all possible modifications due to conjugation of lysine with Cyh-NHS followed by naphthalimide nitroso-diene cycloaddition.

\section{Confocal fluorescence imaging experiments}

A fresh solution of Cyh/Mcp-TPP $(1 \mu \mathrm{M})$ or $2(5 \mu \mathrm{M})$ in DMEM was prepared from $10 \mathrm{mM}$ stock solution in DMSO. The HepG2 cells were incubated with $1 \mu \mathrm{M}$ $\mathrm{Cyh} / \mathrm{Mcp}$-TPP in DMEM at $37{ }^{\circ} \mathrm{C}$ and $5 \% \mathrm{CO}_{2}$ for $60 \mathrm{~min}$. Then the diene-containing medium was removed, and the cells were incubated with $5 \mu \mathrm{M} 2$ in DMEM at $37{ }^{\circ} \mathrm{C}$ and $5 \% \mathrm{CO}_{2}$ for $60 \mathrm{~min}$. The culture was exposed to $365-\mathrm{nm}$ UV light from a handheld UV lamp (UVP, 3UV-38, $8 \mathrm{~W}$, placed on the top of the culture dish at a distance of $1 \mathrm{~cm}$ ) for $0 \mathrm{~min}$ or $5 \mathrm{~min}$, then directly submitted for laser scanning confocal microscopy. The confocal fluorescence images were acquired using Leica TCS SP8 X equipped with $63 \times$ objectiveand appropriate filter as excitation $=405 \mathrm{~nm}$, emission $=450 \sim 580 \mathrm{~nm}$. The control cells were treated with $5 \mu \mathrm{M} 2$ in DMEM in the absence of Cyh/Mcp-TPP.

\section{DFT calculations}


The quantum chemical study was performed with the Gaussian 09 software package $^{12}$. Molecular structure in the ground state for 13-S was obtained by optimizing its geometry at the DFT-B3LYP level with $6-311+\mathrm{G}(\mathrm{d})$ basis set using IEFPCM model to include solvent $\left(\mathrm{CH}_{3} \mathrm{CN}\right)$ effect. Vertical excitation energy and the oscillator strength $(f)$ were computed using time-dependent DFT (TD-DFT) with the $6-311+\mathrm{G}(\mathrm{d})$ basis set. The graphical representation of highest occupied molecular orbitals (HOMO) and the lowest unoccupied molecular orbitals (LUMO) were generated from the corresponding Gaussian cube output files.

\section{References}

[1] C. Hwang, G. Ruan, L. Wang, H. Zheng, E. L. G. Samuel, C. Xiang, W. Lu, W. Kasper, K. Huang, Z. Peng, Z. Schaefer, A. T. Kan, A. A. Martí, M. S. Wong, M. B. Tomson and J. M. Tour, ACS Appl. Mater. Inter. 2014, 6, 7652-7658.

[2] W. Xuan, R. Pan, Y. Cao, K. Liu and W. Wang, Chem. Commun. 2012, 48, 10669-10671.

[3] X. Ao, S. A. Bright, N. C. Taylor and R. B. P. Elmes, Org. Biomol. Chem. 2017, 15, 6104-6108.

[4] A. F. Kluge, B. R. Lagu, P. Maiti, M. Jaleel, M. Webb, J. Malhotra, A. Mallat, P. A. Srinivas, J. E. Thompson, Bioorg. Med. Chem. Lett. 2018, 28, 2655-2659.

[5] B. Li, X. Zhou, P. Yang, L. Zhu, Y. Zhong, Z. Cai, B. Jiang, X. Cai, J. Liu* and X. Jiang, Adv. Sci. 2019, 6, 1802039.

[6] W. Lin, A. Gupta, K. H. Kim, D. Mendel and M. J. Miller, Org. Lett. 2009, 11, 449-452.

[7] V. Steven and D. Graham, Org. Biomol. Chem. 2008, 6, 3781-3787.

[8] G. I. Peterson, D. C. Church, N. A. Yakelis and A. J. Boydston, Polymer 2014, 55, 5980-5985.

[9] Oxford Diffraction, Xcalibur CCD System. CrysAlisPro. Oxford Diffraction Ltd: Abingdon, England, UK, 201024.

[10] O. V. Dolomanov, L. J. Bourhis, R. J. Gildea, J. A. K. Howard, H. Puschmann, J. Appl. Crystallogr. 2009, 42, 339-341.

[11] D. Kratzert, J. J. Holstein, I. Krossing, J. Appl. Crystallogr. 2015, 48, 933-938.

[12] Gaussian 09, Revision D.01, M. J. Frisch, G. W. Trucks, H. B. Schlegel, G. E. Scuseria, M.A. Robb, J. R. Cheeseman, G. Scalmani, V. Barone, B. Mennucci, G. A. Petersson, H.Nakatsuji, M. Caricato, X. Li, H. P. Hratchian, A. F. Izmaylov, J. Bloino, G. Zheng, J. L.Sonnenberg, M. Hada, M. Ehara, K. Toyota, R. Fukuda, J. Hasegawa, M. Ishida, T.Nakajima, Y. Honda, O. Kitao, H. Nakai, T. Vreven, J. A. Montgomery, Jr., J. E. Peralta, F.Ogliaro, M. Bearpark, J. J. Heyd, E. Brothers, K. N. Kudin, V. N. Staroverov, R.Kobayashi, J. Normand, K. Raghavachari, A. Rendell, J. C. Burant, S. S. Iyengar, J.Tomasi, M. Cossi, N. Rega, J. M. Millam, M. Klene, J. E. Knox, J. B. Cross, V. Bakken, C.Adamo, J. Jaramillo, R. Gomperts, R. E. Stratmann, O. Yazyev, A. J. Austin, R. Cammi, C.Pomelli, J. W. Ochterski, R. L. Martin, K. Morokuma, V. G. Zakrzewski, G. A. Voth, P.Salvador, J. J. Dannenberg, S. Dapprich, A. D. Daniels, Ö. Farkas, J. B. Foresman, J. V.Ortiz, J. Cioslowski, and D. J. Fox, Gaussian, Inc., Wallingford CT, 2009. 
${ }^{1} \mathrm{H}$ NMR of compound $\mathbf{S} 1\left(400 \mathrm{MHz}\right.$ in $\mathrm{CDCl}_{3}$ )

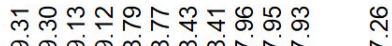
कं

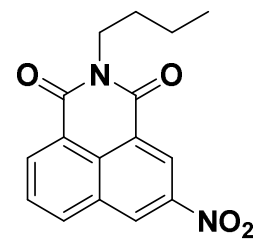

S1
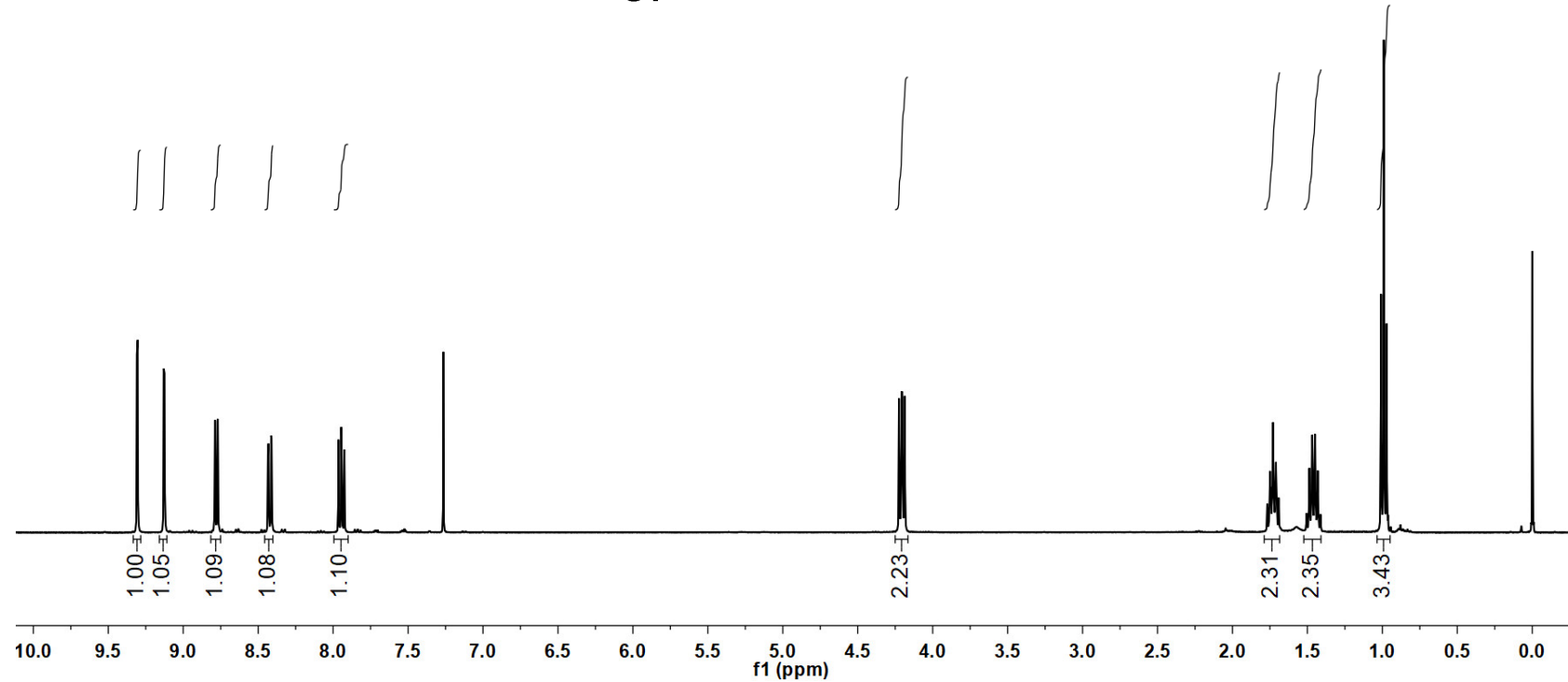

${ }^{13} \mathrm{C}$ NMR of compound $\mathbf{S 1}\left(101 \mathrm{MHz}\right.$ in $\left.\mathrm{CDCl}_{3}\right)$
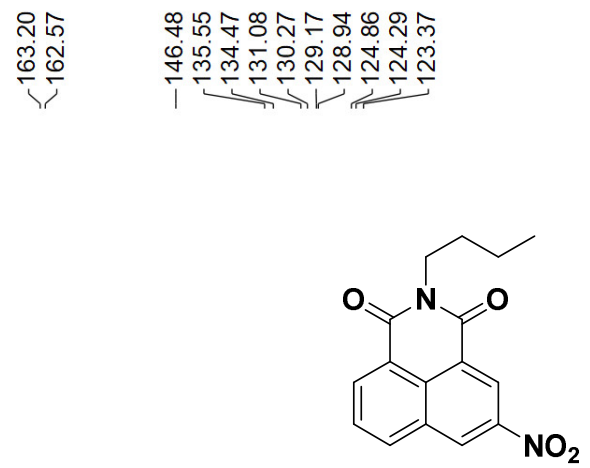

S1

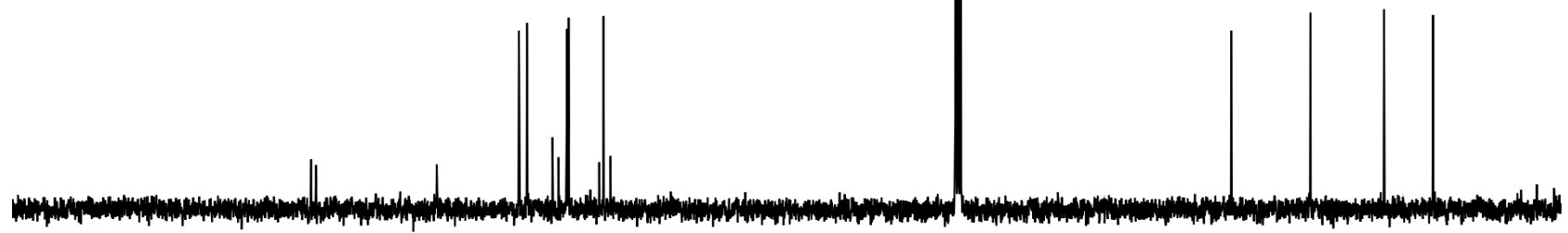


${ }^{1} \mathrm{H}$ NMR of compound $1\left(400 \mathrm{MHz}\right.$ in $\left.\mathrm{CDCl}_{3}\right)$

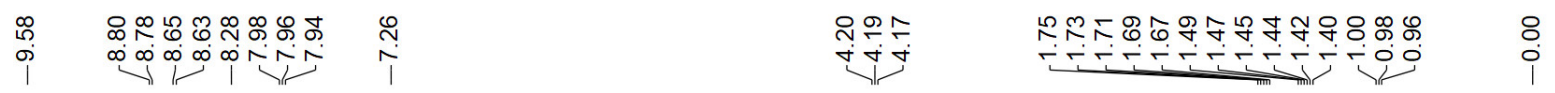<smiles>CCCCN1C(=O)c2cccc3cc([N+](=O)[O-])cc(c23)C1=O</smiles>

1

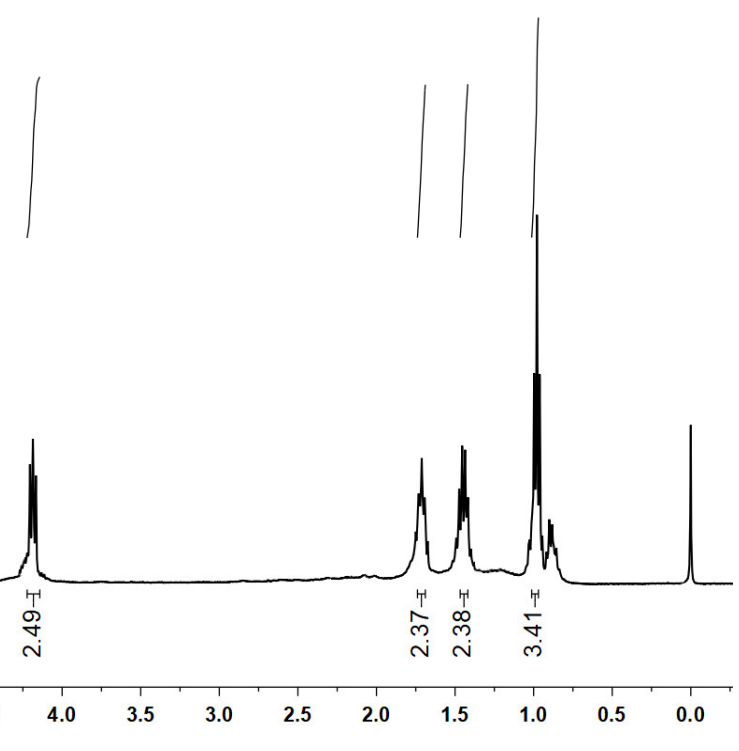

${ }^{13} \mathrm{C}$ NMR of compound $\mathbf{1}\left(101 \mathrm{MHz}\right.$ in $\left.\mathrm{CDCl}_{3}\right)$
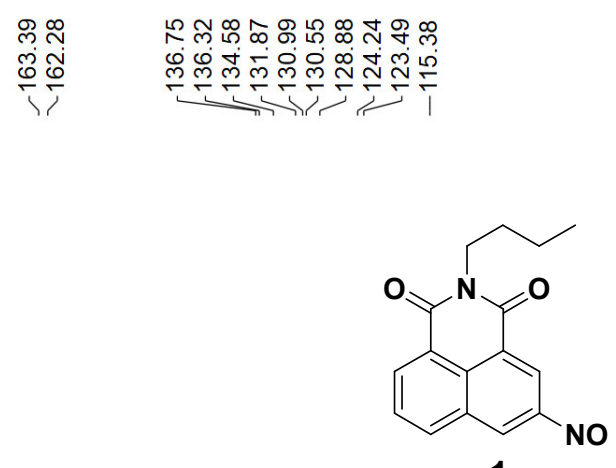

1

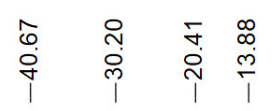


${ }^{1} \mathrm{H}$ NMR of compound $2\left(400 \mathrm{MHz}\right.$ in $\left.\mathrm{CDCl}_{3}\right)$

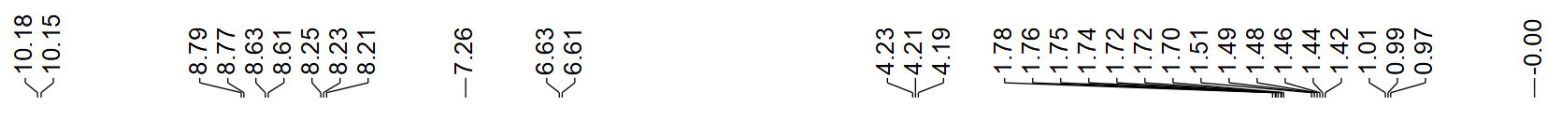<smiles>CCCCN1C(=O)c2cccc3c([N+](=O)[O-])ccc(c23)C1=O</smiles><smiles>C=CC(=C)C</smiles><smiles>C=CCC</smiles>

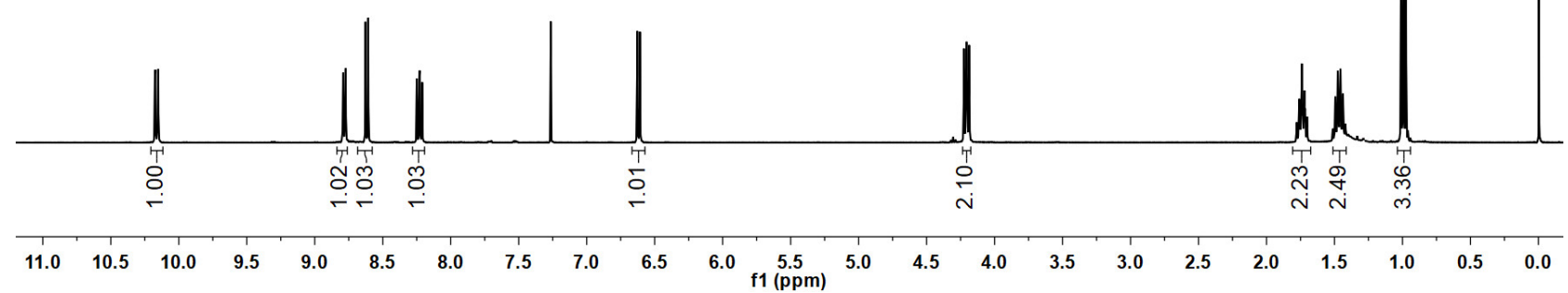

${ }^{13} \mathrm{C}$ NMR of compound $2\left(101 \mathrm{MHz}\right.$ in $\left.\mathrm{CDCl}_{3}\right)$

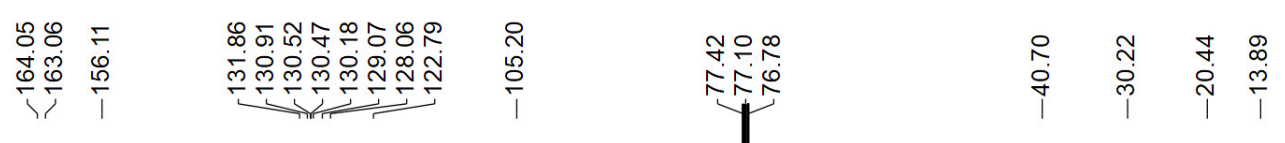

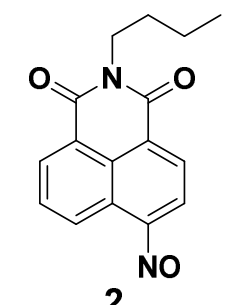

200 $\begin{array}{llllllllll}190 & 180 & 170 & 160 & 150 & 140 & 130 & 120 & 110 & \begin{array}{c}100 \\ \mathrm{f} 1(\mathrm{ppm})\end{array}\end{array}$ 
${ }^{1} \mathrm{H}$ NMR of compound $\mathbf{S 4}\left(400 \mathrm{MHz}\right.$ in $\left.\mathrm{CDCl}_{3}\right)$

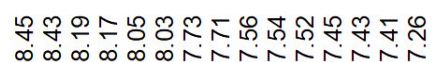

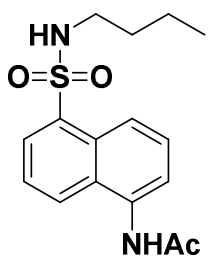

S4

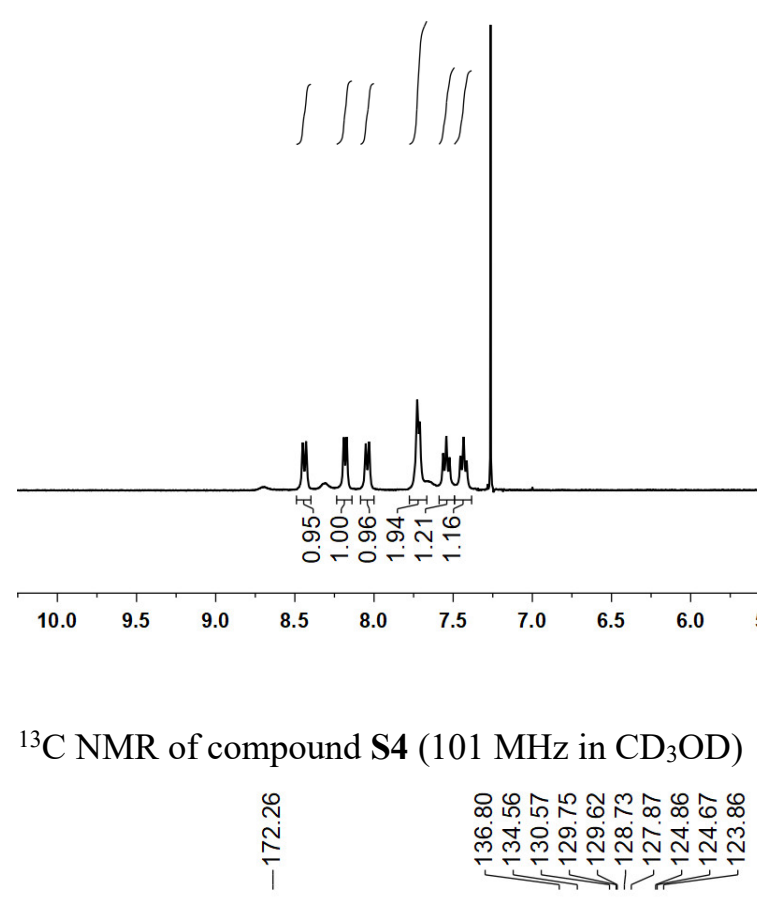

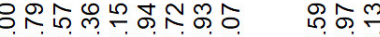

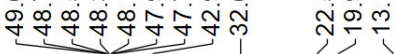

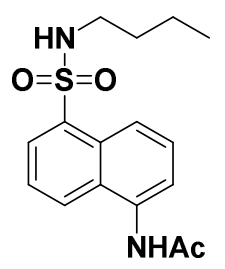

S4 
${ }^{1} \mathrm{H}$ NMR of compound $\mathbf{S 5}\left(400 \mathrm{MHz}\right.$ in $\mathrm{CDCl}_{3}$ )

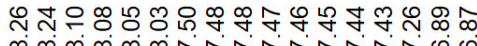

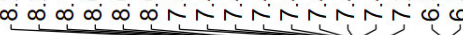<smiles>CCCCNS(=O)(=O)c1cccc2c(N)cccc12</smiles>

S5
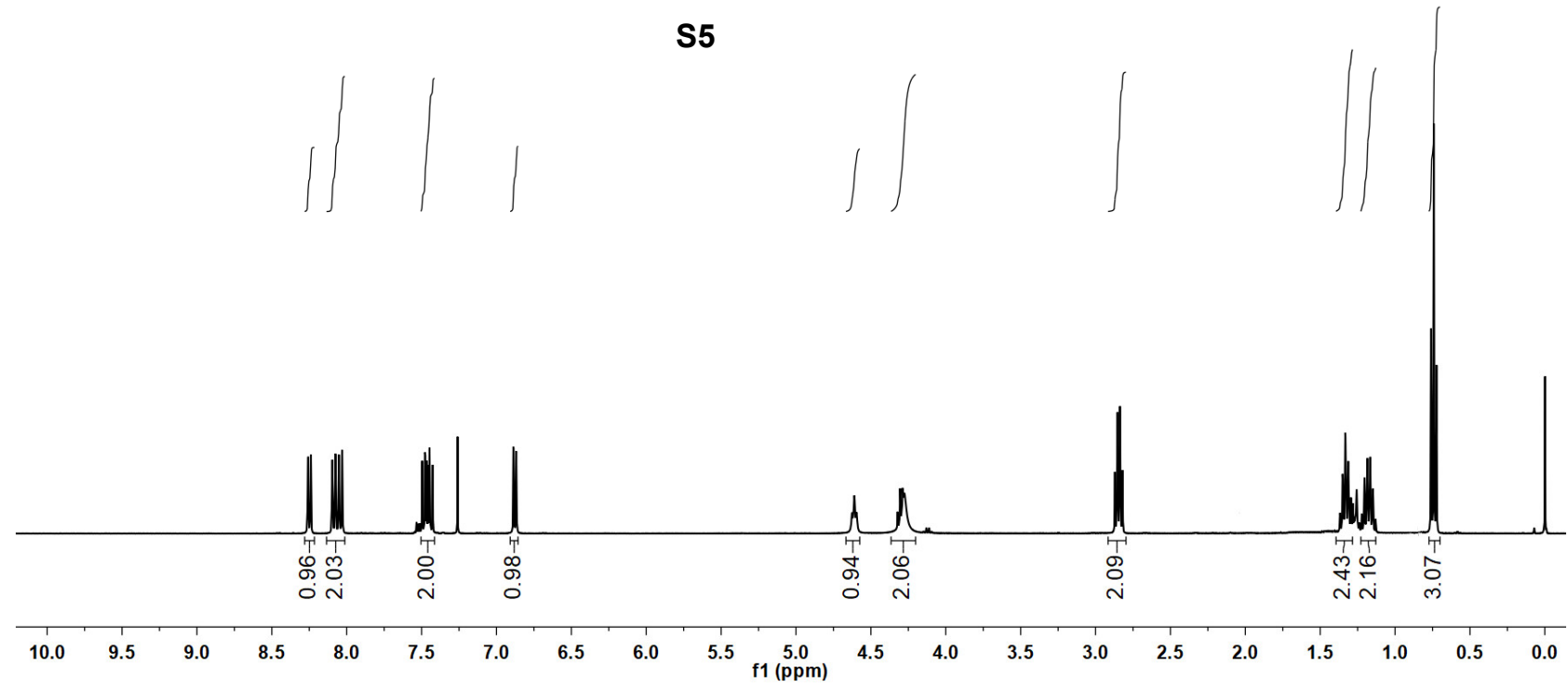

${ }^{13} \mathrm{C}$ NMR of compound $\mathbf{S 5}$ (101 $\mathrm{MHz}$ in $\mathrm{CDCl}_{3}$ )

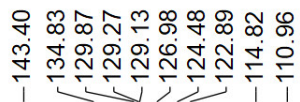

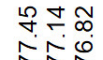

Nヘ̃

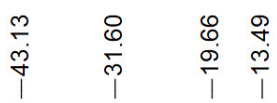

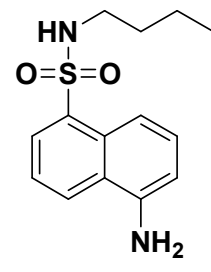

S5

200

$\begin{array}{lllllllll}190 & 180 & 170 & 160 & 150 & 140 & 130 & 120 & 110 \begin{array}{c}100 \\ \mathrm{f} 1(\mathrm{ppm})\end{array}\end{array}$


${ }^{1} \mathrm{H}$ NMR of compound $3\left(400 \mathrm{MHz}\right.$ in $\mathrm{CDCl}_{3}$ )

它<smiles>CCCCNS(=O)(=O)c1cccc2c([N+](=O)[O-])cccc12</smiles>

3

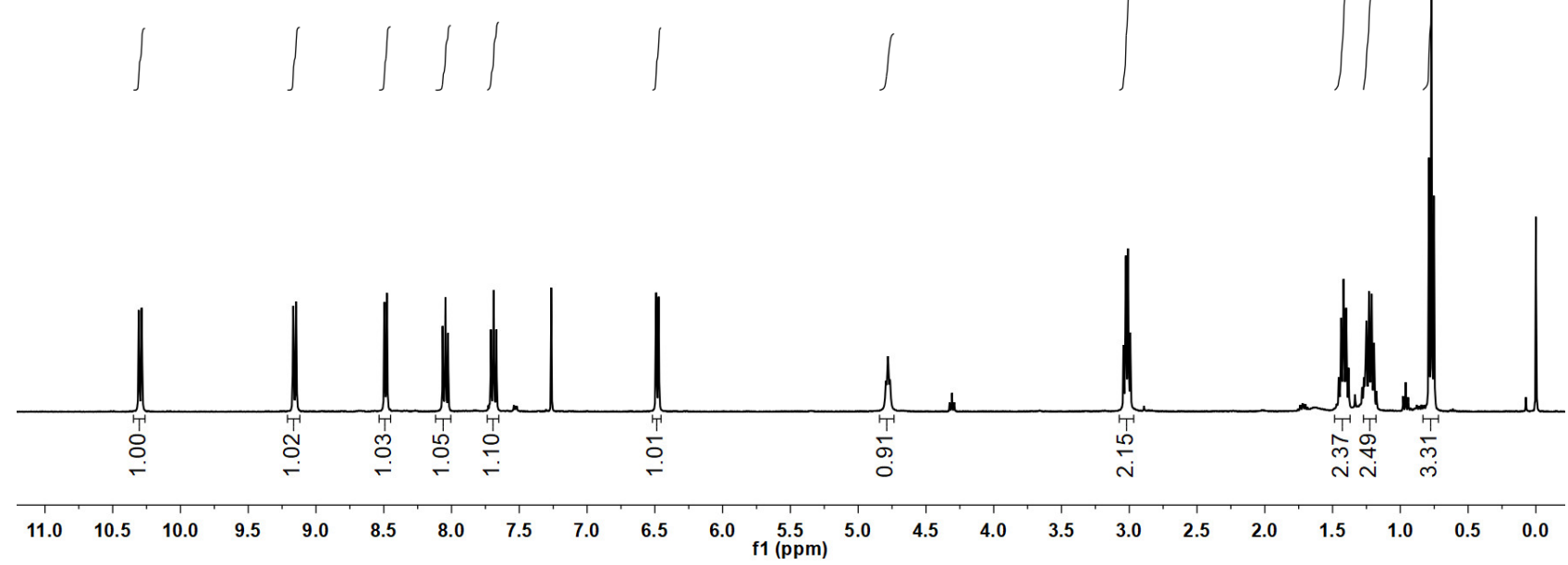

${ }^{13} \mathrm{C}$ NMR of compound $3\left(101 \mathrm{MHz}\right.$ in $\left.\mathrm{CDCl}_{3}\right)$

\begin{tabular}{|c|c|c|c|c|}
\hline 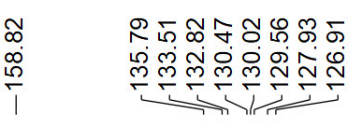 & $\begin{array}{l}\stackrel{0}{0} \\
\stackrel{0}{0} \\
i\end{array}$ & 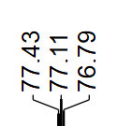 & $\stackrel{\infty}{\stackrel{\rho}{j}}$ & 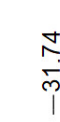 \\
\hline
\end{tabular}

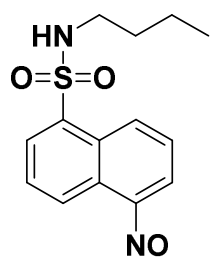

3

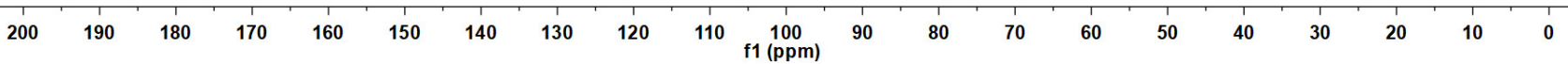


${ }^{1} \mathrm{H}$ NMR of compound $4\left(600 \mathrm{MHz}\right.$ in $\left.\mathrm{CDCl}_{3}\right)$

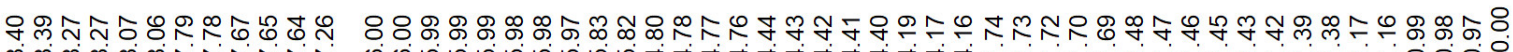
o

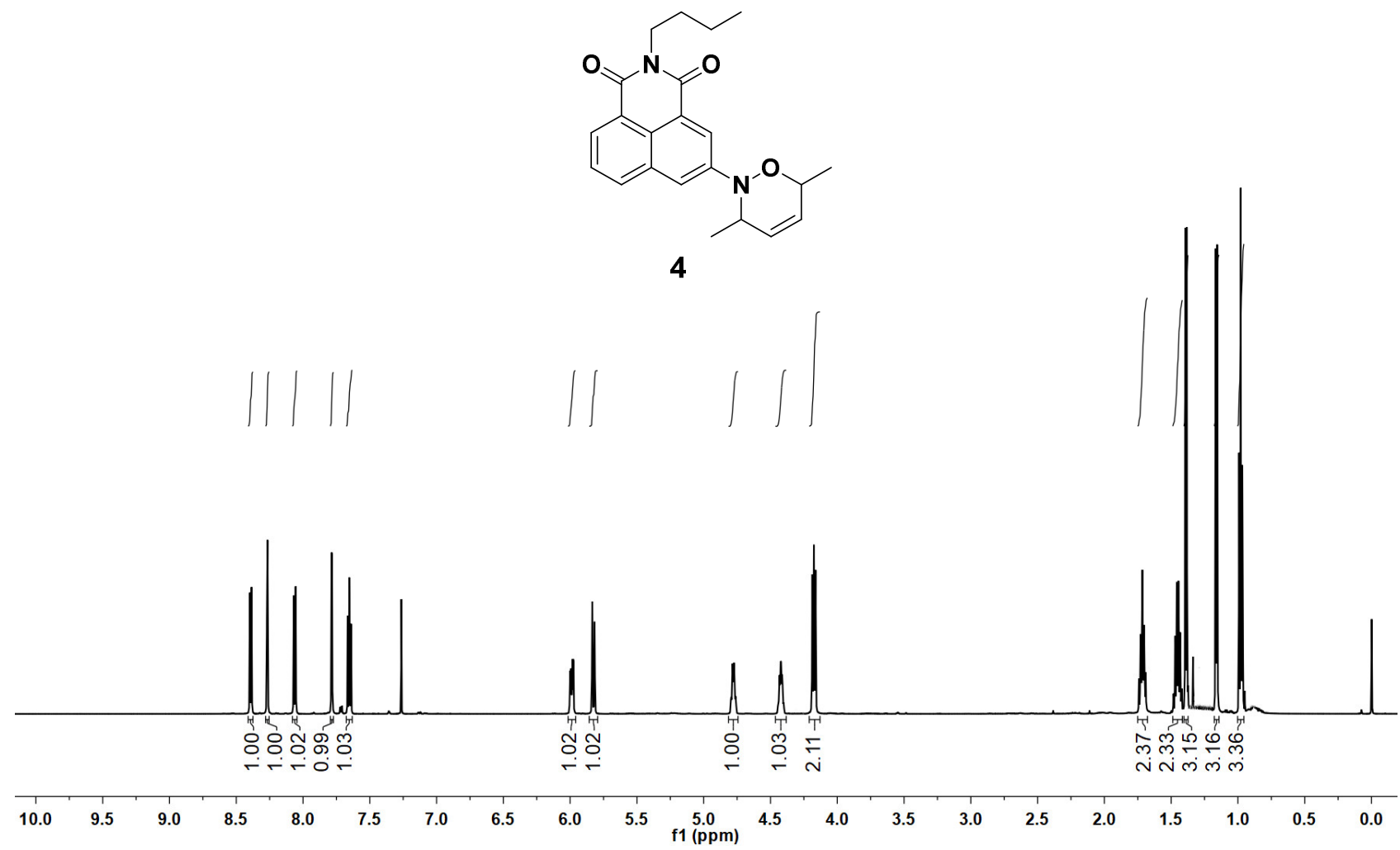

${ }^{13} \mathrm{C}$ NMR of compound $4\left(151 \mathrm{MHz}\right.$ in $\left.\mathrm{CDCl}_{3}\right)$

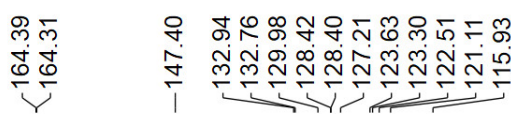

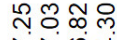

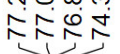

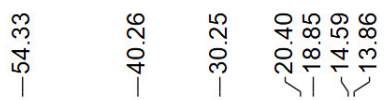

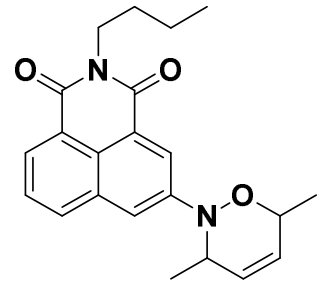

4

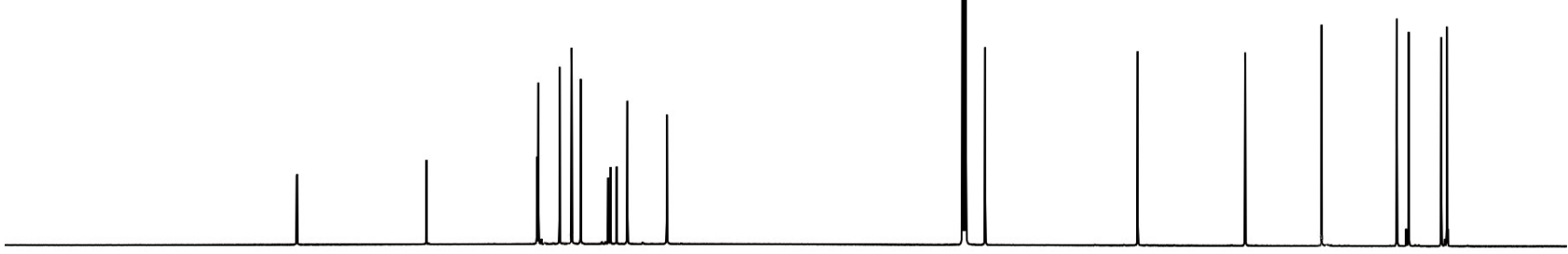

200

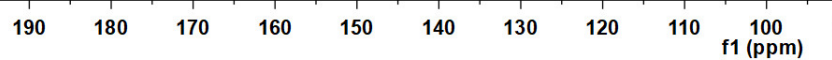


${ }^{1} \mathrm{H}$ NMR of compound $5\left(600 \mathrm{MHz}\right.$ in $\left.\mathrm{CDCl}_{3}\right)$

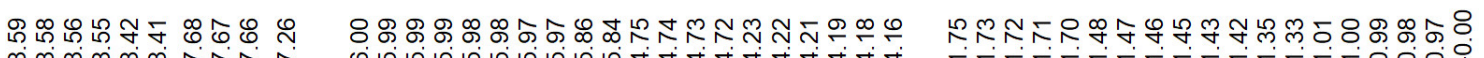

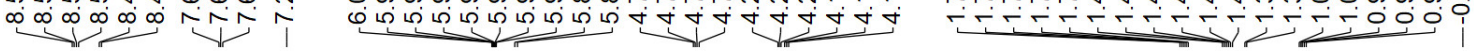<smiles>CCCCN1C(=O)c2cccc3c(N4OC(C)C=CC4C)ccc(c23)C1=O</smiles>

5

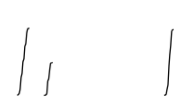

$\iint$ 

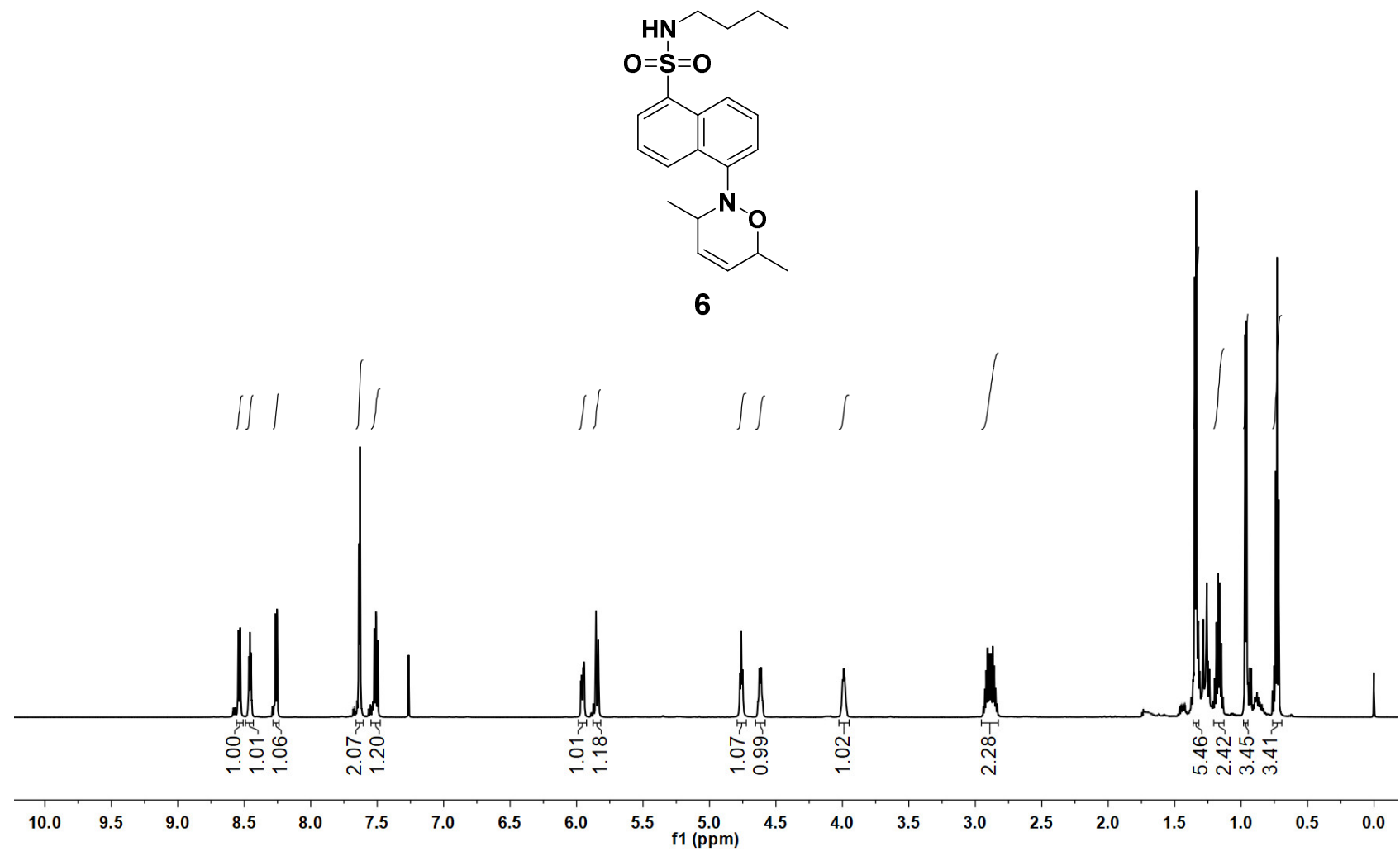

${ }^{13} \mathrm{C}$ NMR of compound $6\left(151 \mathrm{MHz}\right.$ in $\left.\mathrm{CDCl}_{3}\right)$

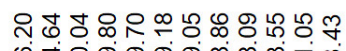

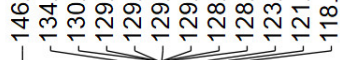

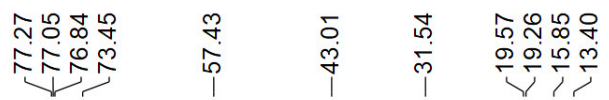

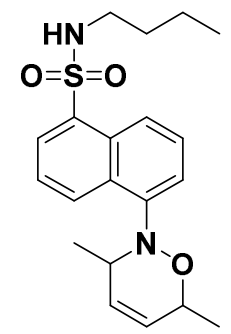

6

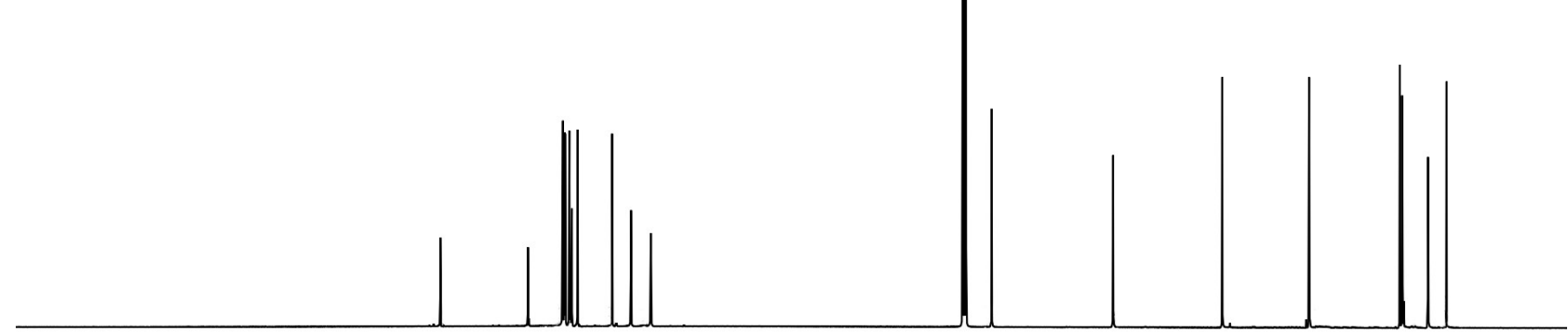


${ }^{1} \mathrm{H}$ NMR of compound $7\left(400 \mathrm{MHz}\right.$ in $\left.\mathrm{CDCl}_{3}\right)$

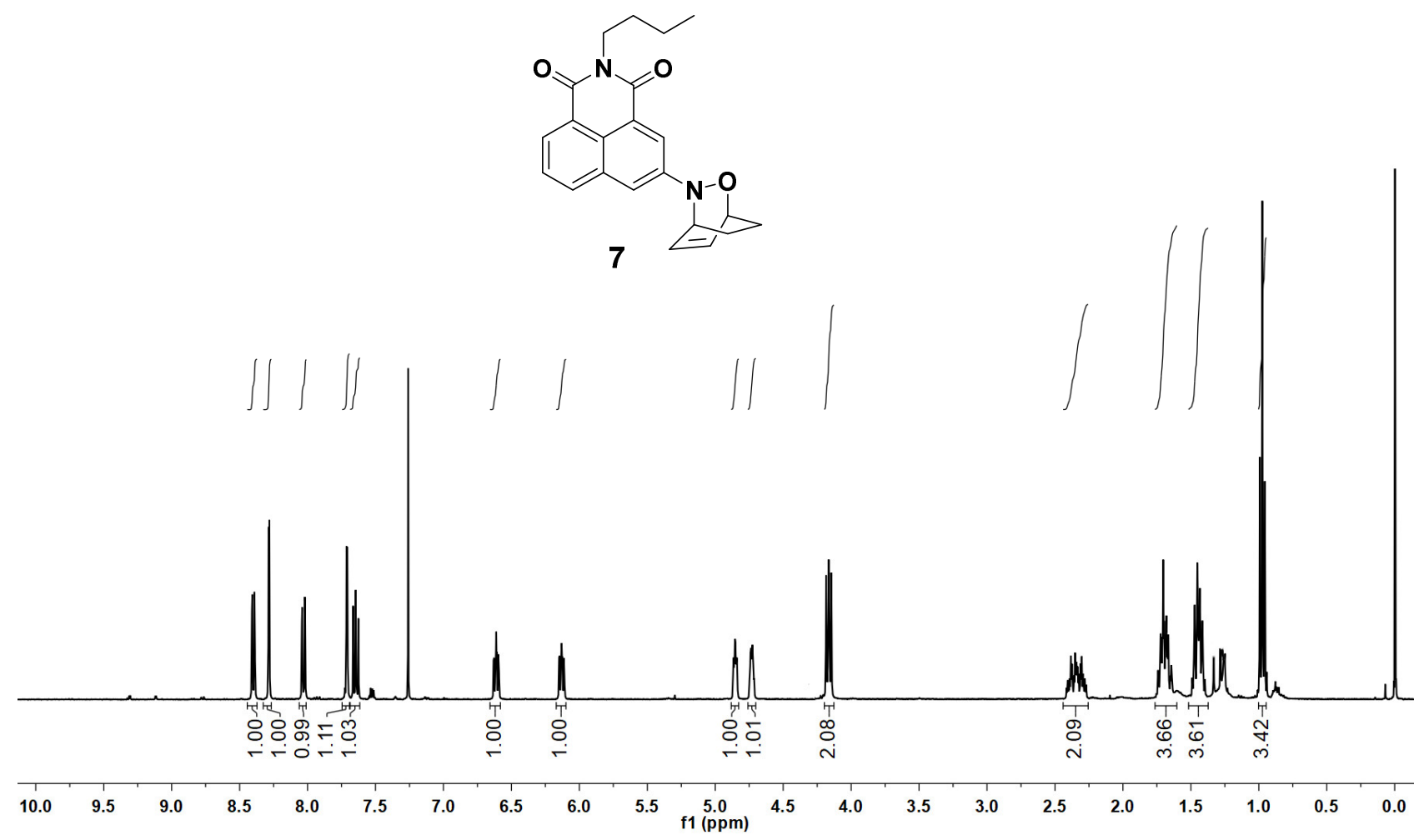

${ }^{13} \mathrm{C}$ NMR of compound $7\left(101 \mathrm{MHz}\right.$ in $\left.\mathrm{CDCl}_{3}\right)$
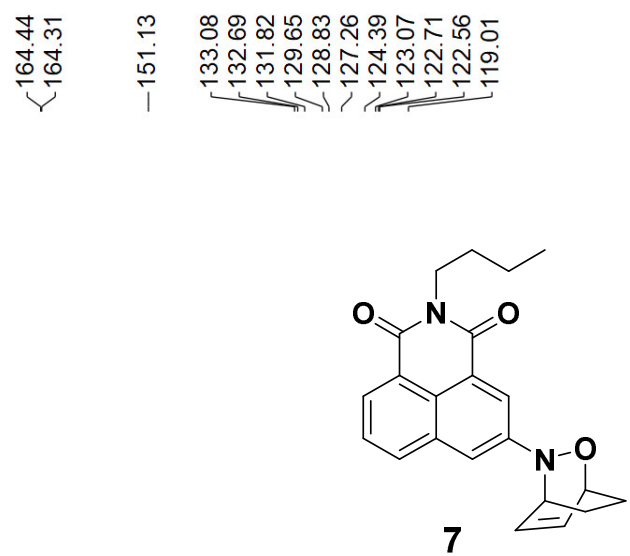
${ }^{1} \mathrm{H}$ NMR of compound $8\left(400 \mathrm{MHz}\right.$ in $\left.\mathrm{CDCl}_{3}\right)$

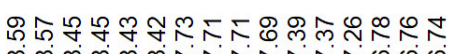

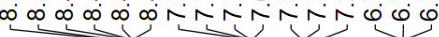

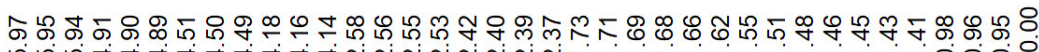
nin<smiles>CCCCN1C(=O)c2cccc3c(N4OC5CC4C5)ccc(c23)C1=O</smiles><smiles>c1ccccc1</smiles><smiles>[C]1CCC2CC1C2</smiles>

8
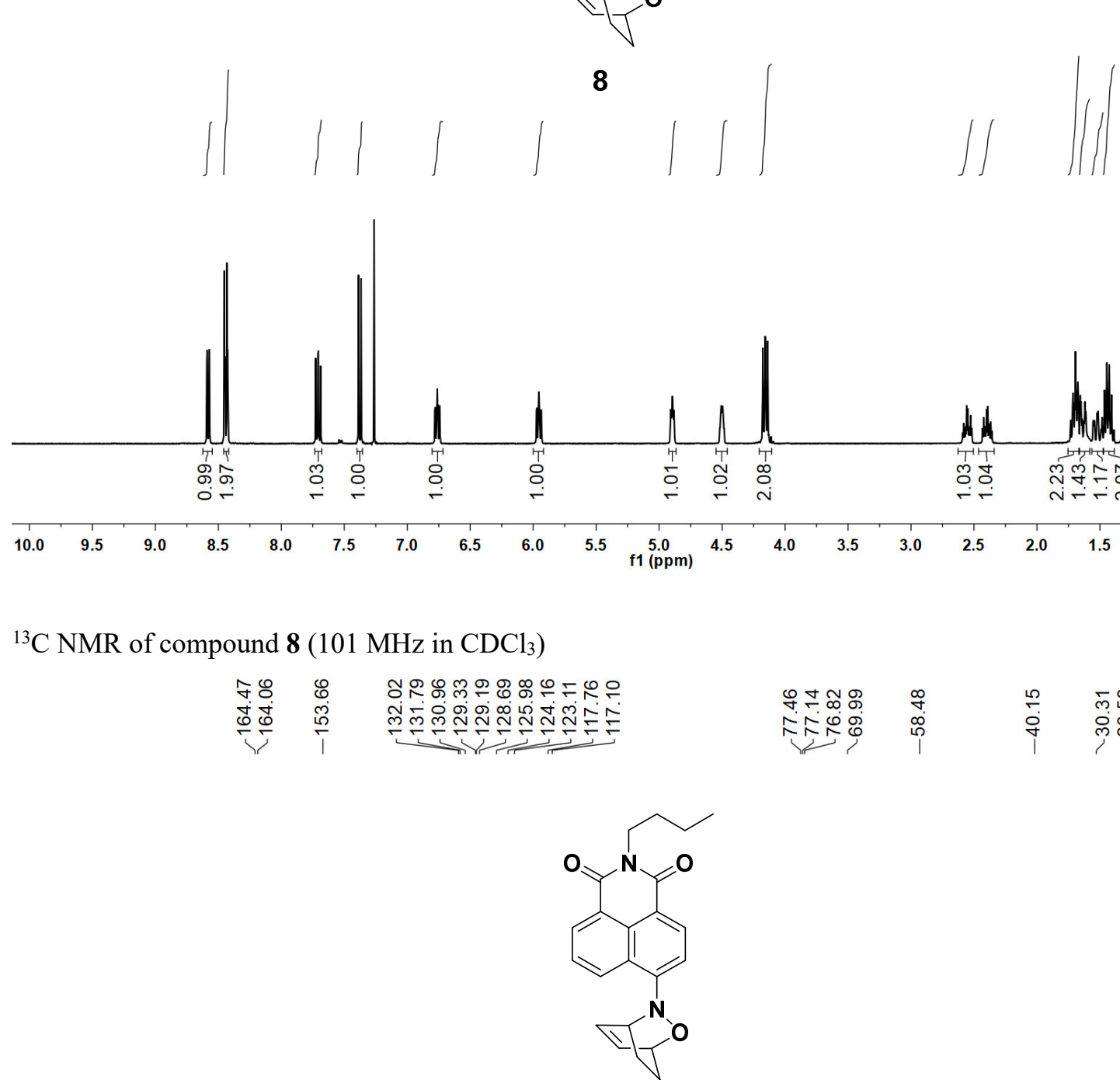

8

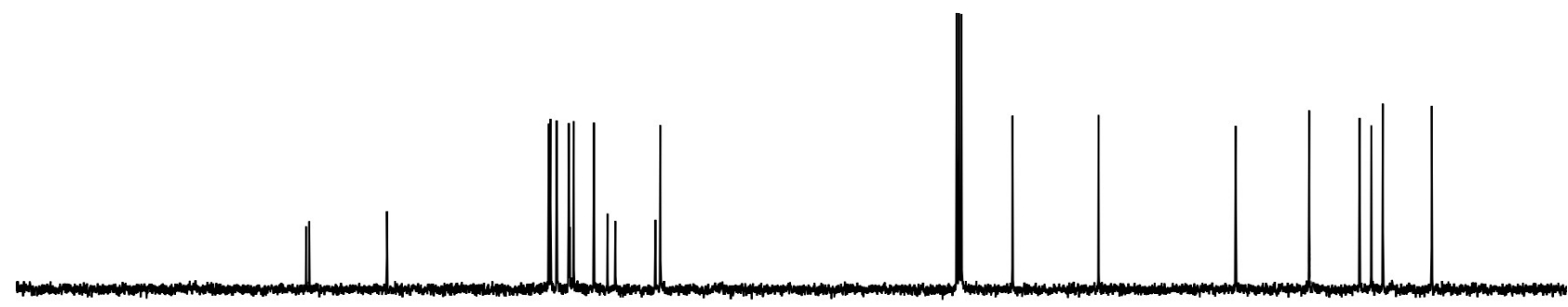


${ }^{1} \mathrm{H}$ NMR of compound $9\left(400 \mathrm{MHz}\right.$ in $\left.\mathrm{CDCl}_{3}\right)$

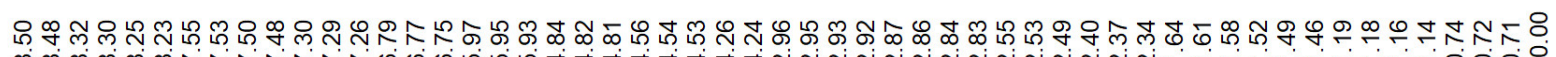

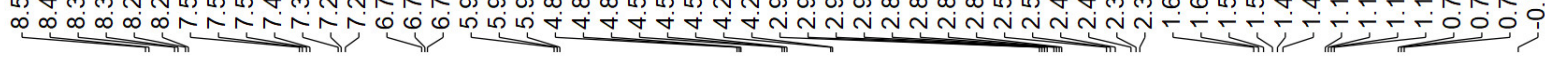

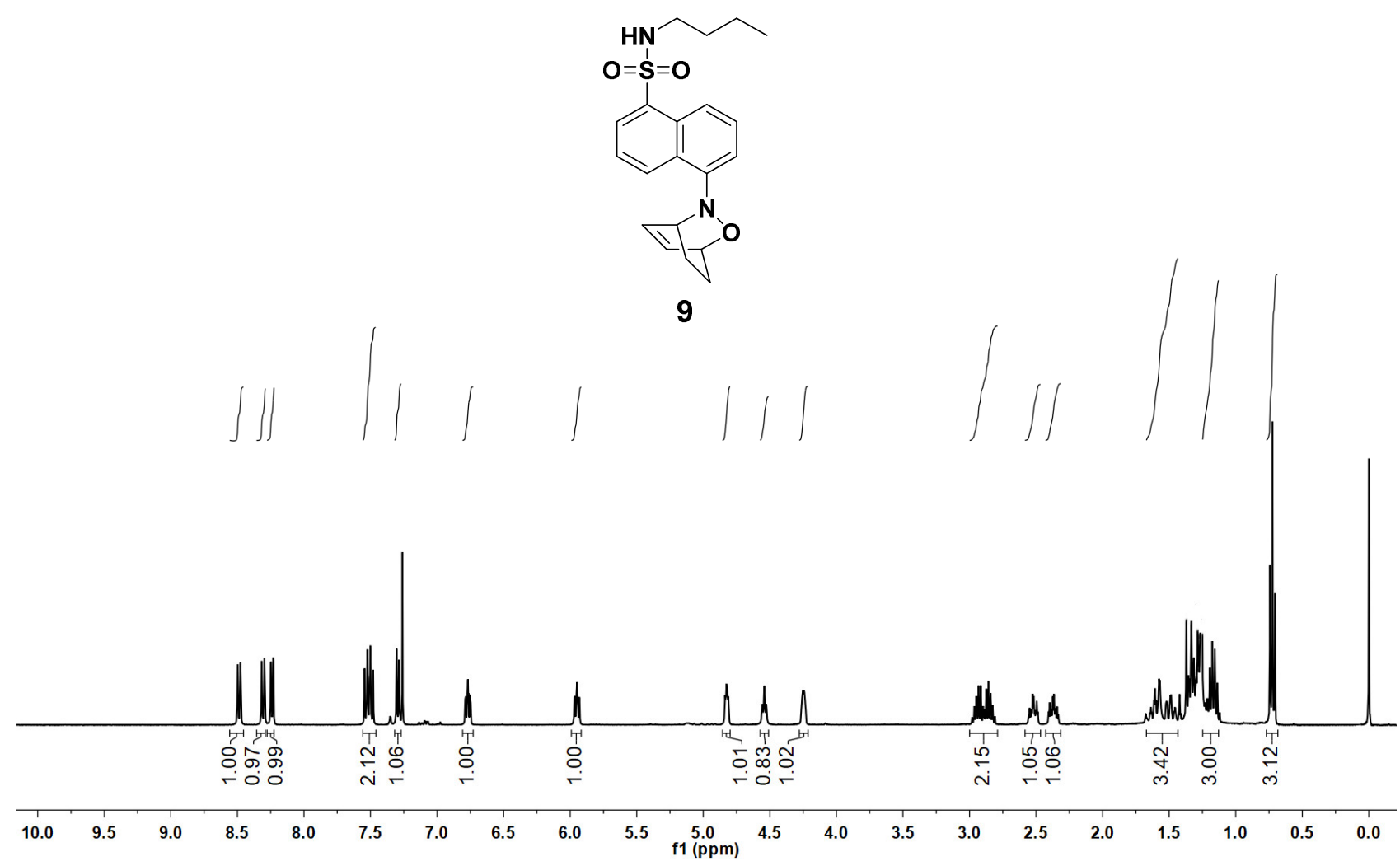

${ }^{13} \mathrm{C}$ NMR of compound $9\left(101 \mathrm{MHz}\right.$ in $\left.\mathrm{CDCl}_{3}\right)$

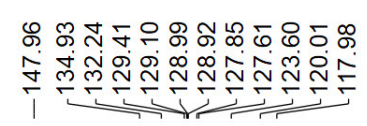

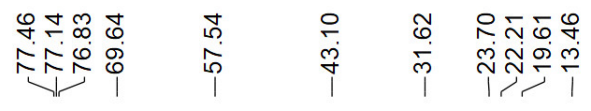

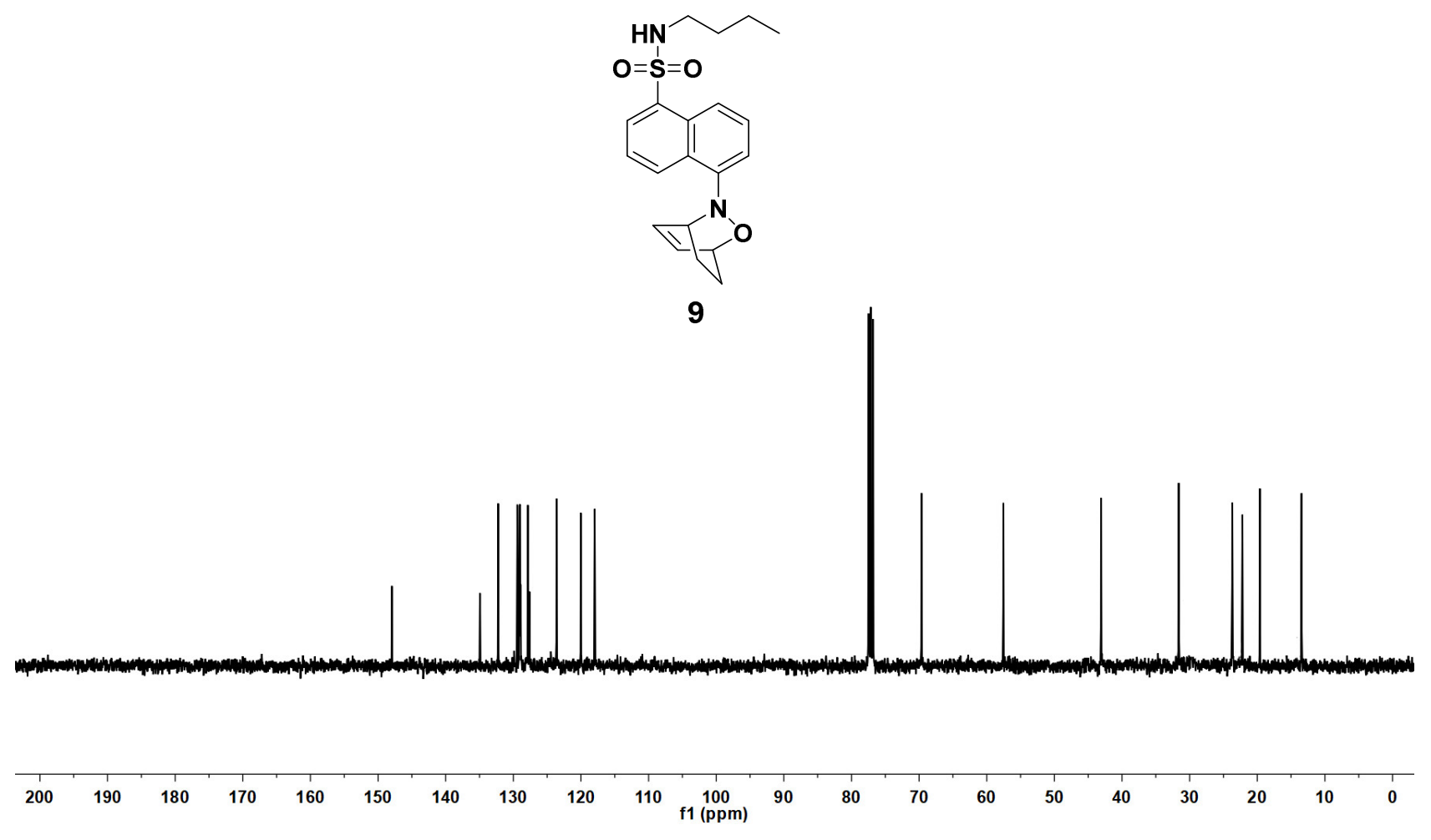




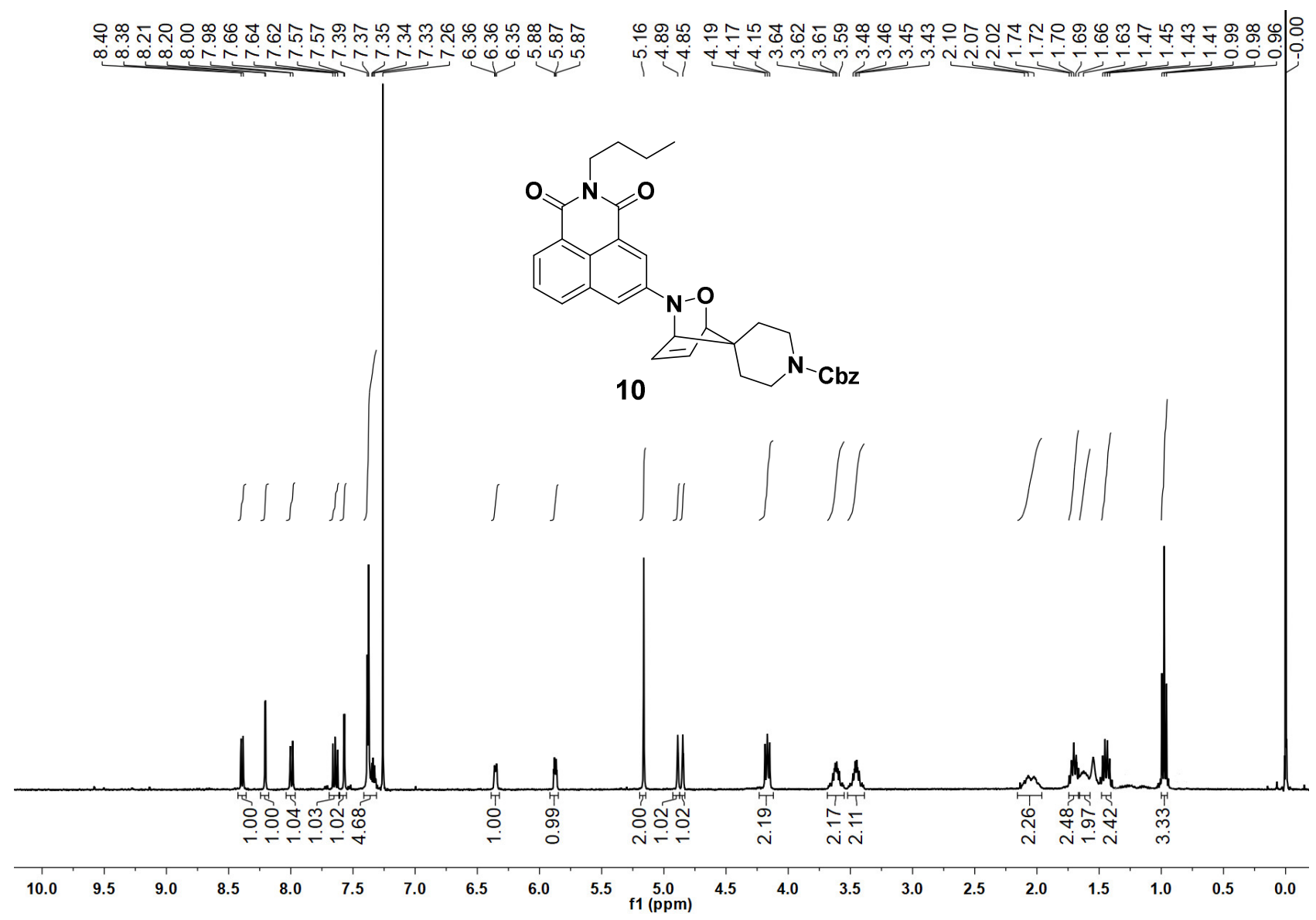

${ }^{13} \mathrm{C}$ NMR of compound $10\left(101 \mathrm{MHz}\right.$ in $\left.\mathrm{CDCl}_{3}\right)$
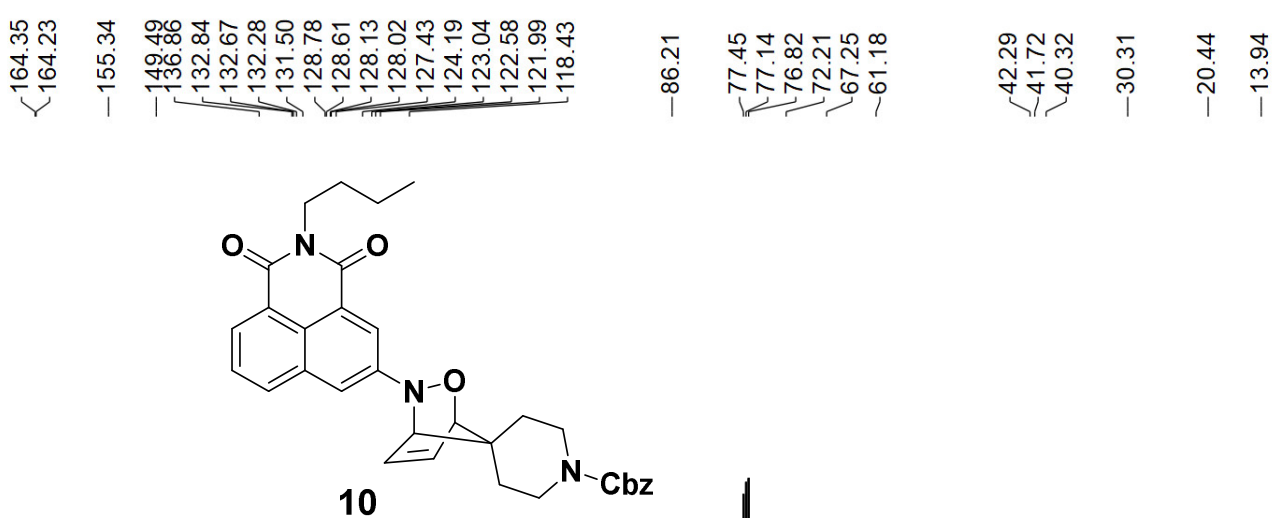

10

bz 
${ }^{1} \mathrm{H}$ NMR of compound $11\left(400 \mathrm{MHz}\right.$ in $\left.\mathrm{CDCl}_{3}\right)$

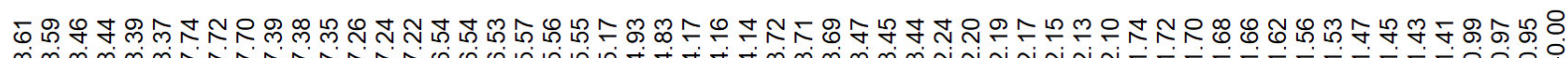

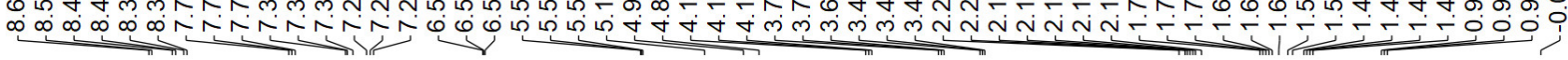

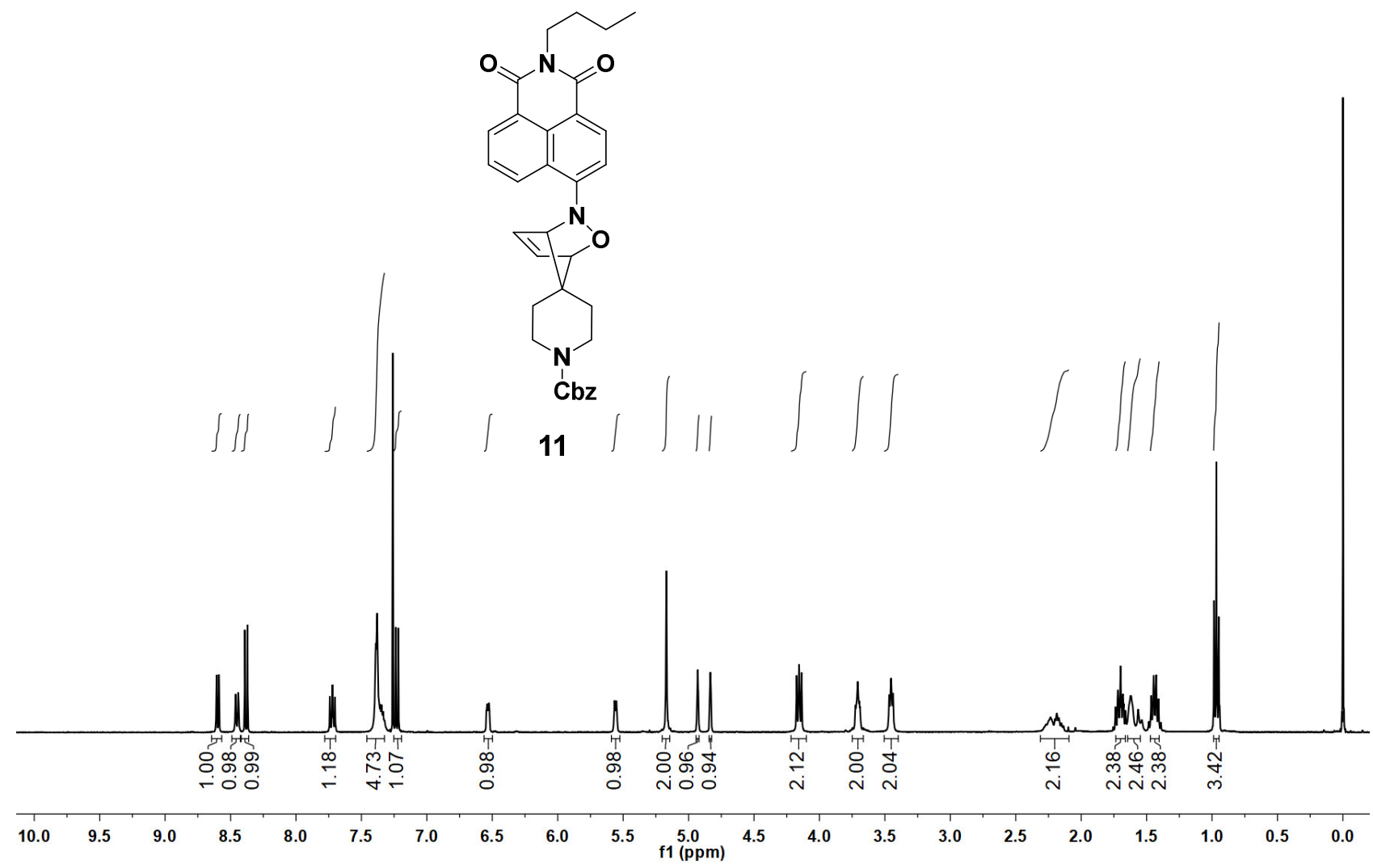

${ }^{13} \mathrm{C}$ NMR of compound $11\left(101 \mathrm{MHz}\right.$ in $\left.\mathrm{CDCl}_{3}\right)$

D象

它家

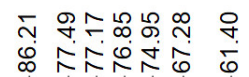

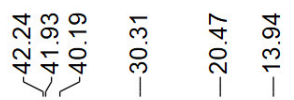

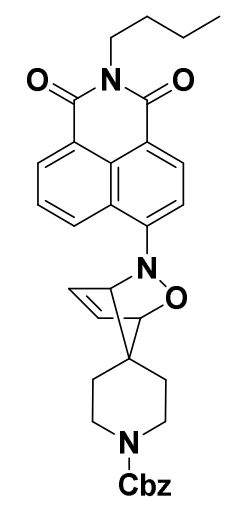

11

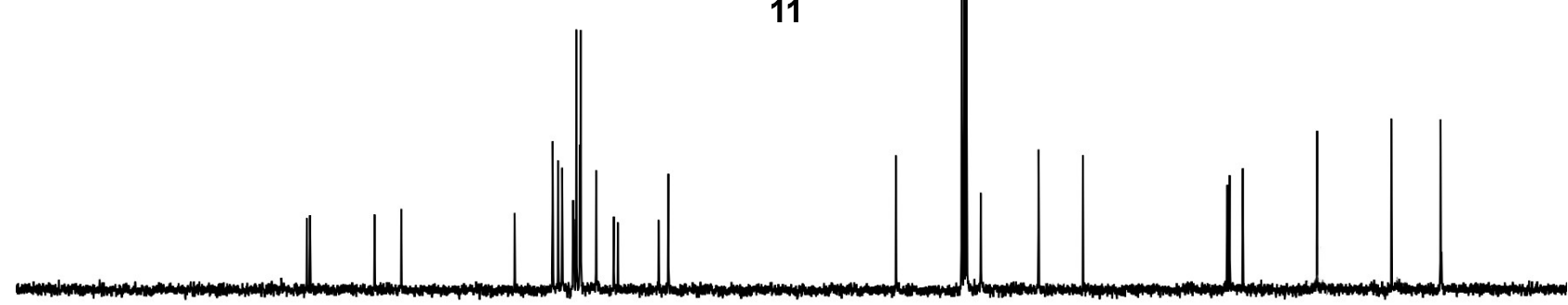

200

$\begin{array}{lllllllll}190 & 180 & 170 & 160 & 150 & 140 & 130 & 120 & 110 \begin{array}{c}100 \\ \mathrm{f} 1(\mathrm{ppm})\end{array}\end{array}$ 
${ }^{1} \mathrm{H}$ NMR of compound $12\left(400 \mathrm{MHz}\right.$ in $\left.\mathrm{CDCl}_{3}\right)$

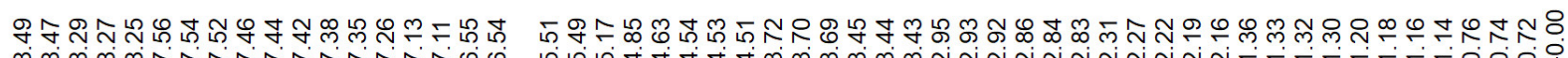

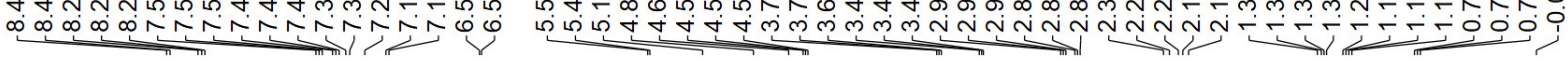

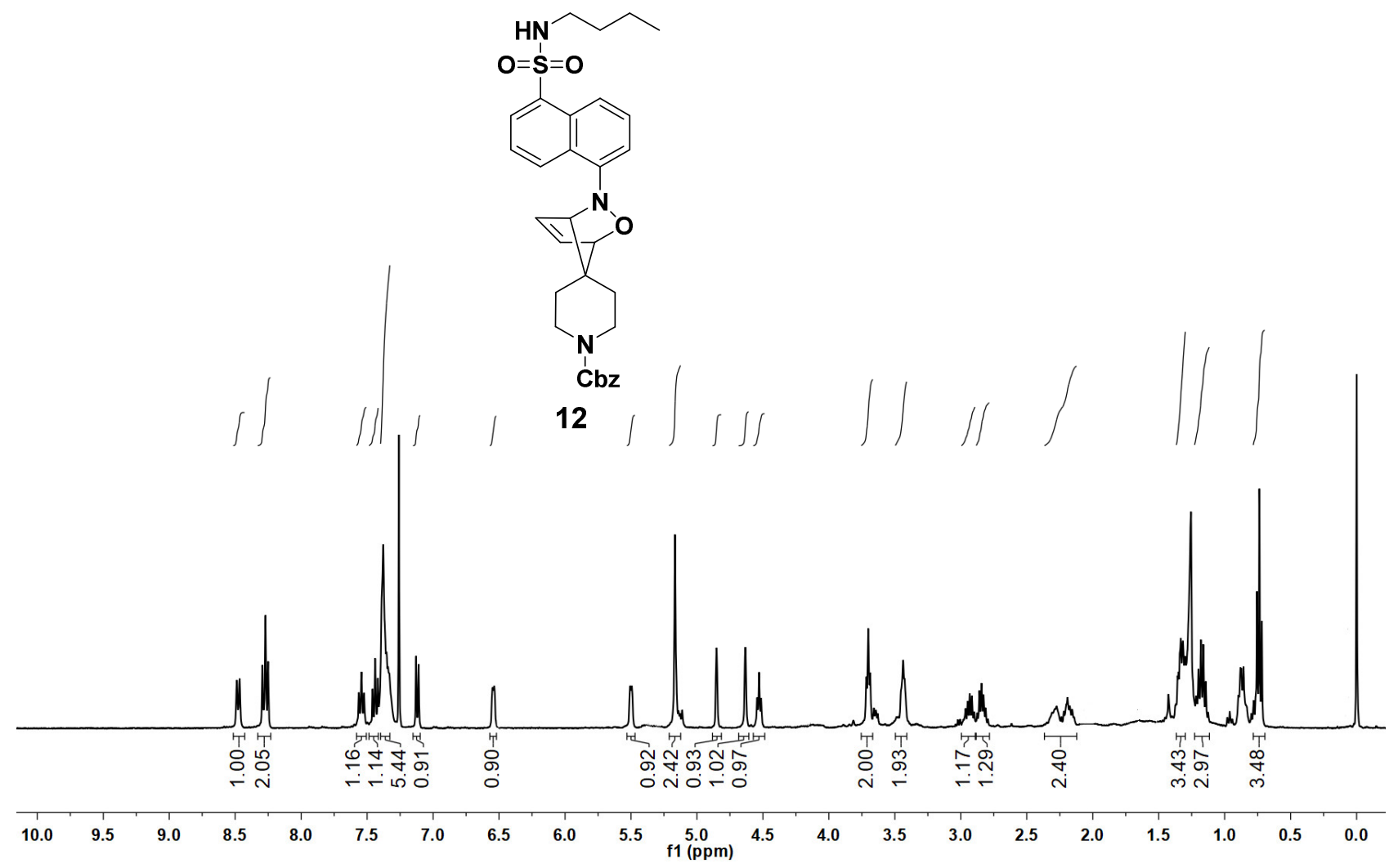

${ }^{13} \mathrm{C}$ NMR of compound $12\left(101 \mathrm{MHz}\right.$ in $\left.\mathrm{CDCl}_{3}\right)$

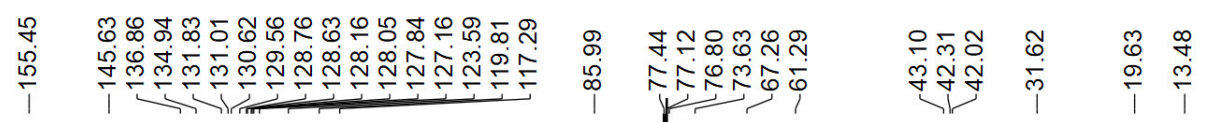

$\begin{array}{llllllllllllllllllll}1 & 1 & 1 & 1 & 1 & 1 & 1 & 100 & 90 & 80 & 70 & 60 & 50 & 40 & 30 & 20 & 10 & 0\end{array}$ 
${ }^{1} \mathrm{H}$ NMR of compound $13\left(600 \mathrm{MHz}\right.$ in $\left.\mathrm{CD}_{3} \mathrm{CN}\right)$

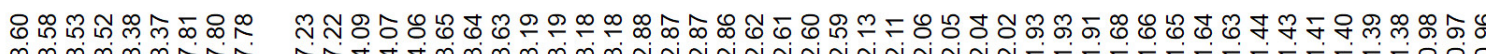

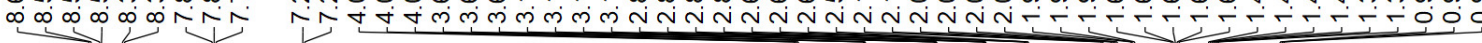

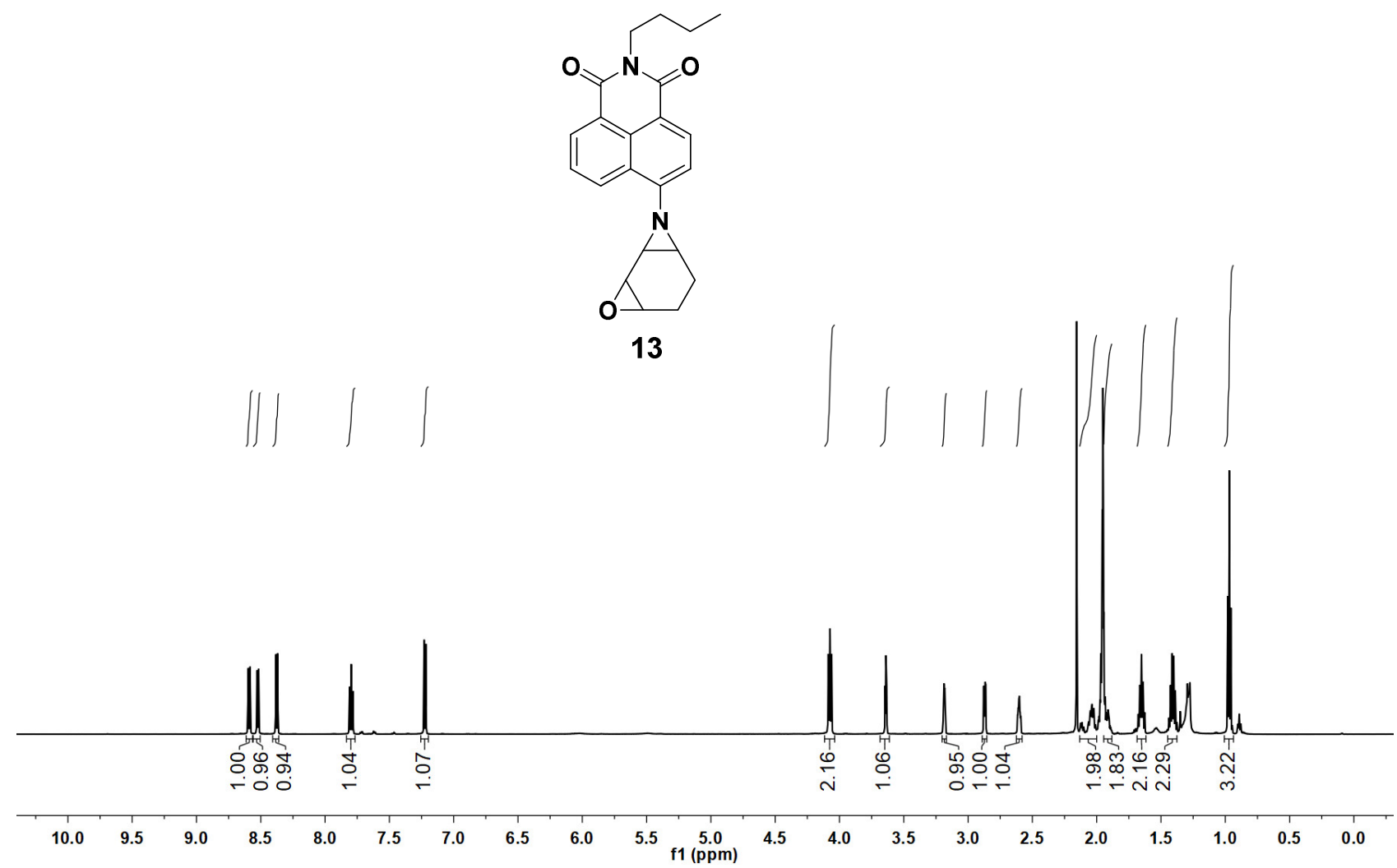

${ }^{13} \mathrm{C}$ NMR of compound $\mathbf{1 3}\left(151 \mathrm{MHz}\right.$ in $\left.\mathrm{CD}_{3} \mathrm{CN}\right)$

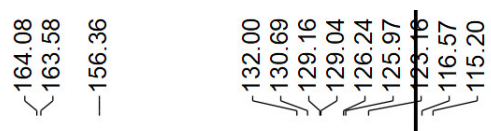

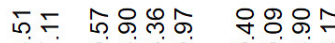

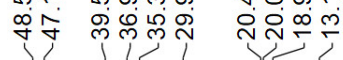

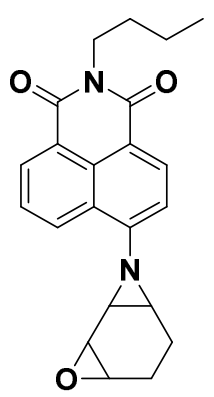

13

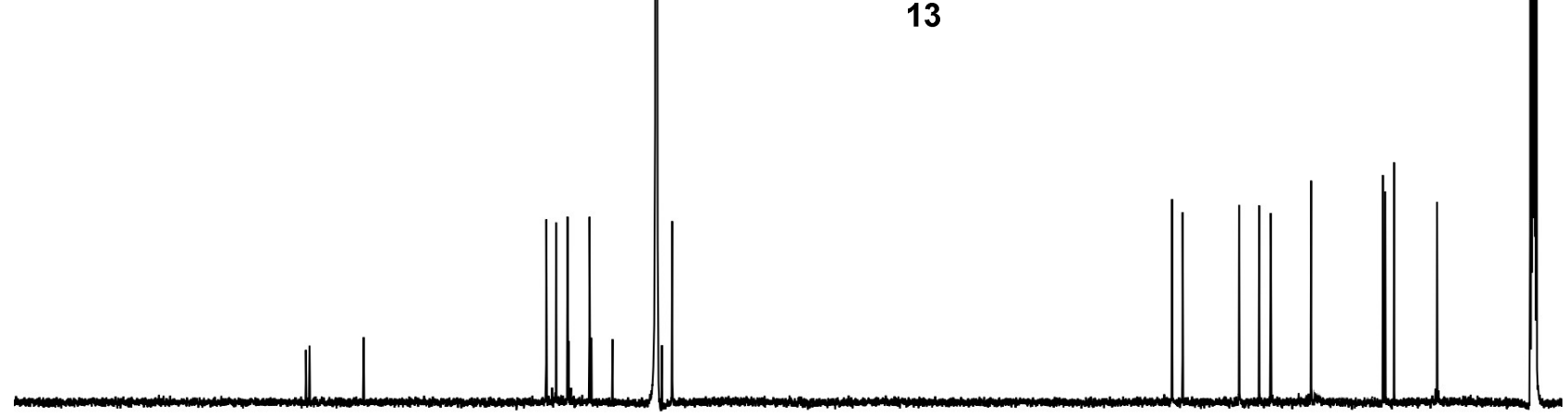

200

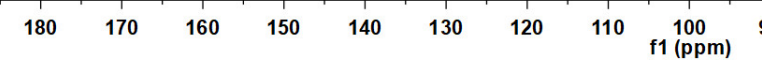


${ }^{1} \mathrm{H}$ NMR of compound $14\left(600 \mathrm{MHz}\right.$ in $\left.\mathrm{CDCl}_{3}\right)$

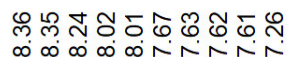

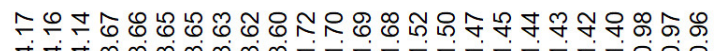

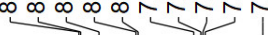
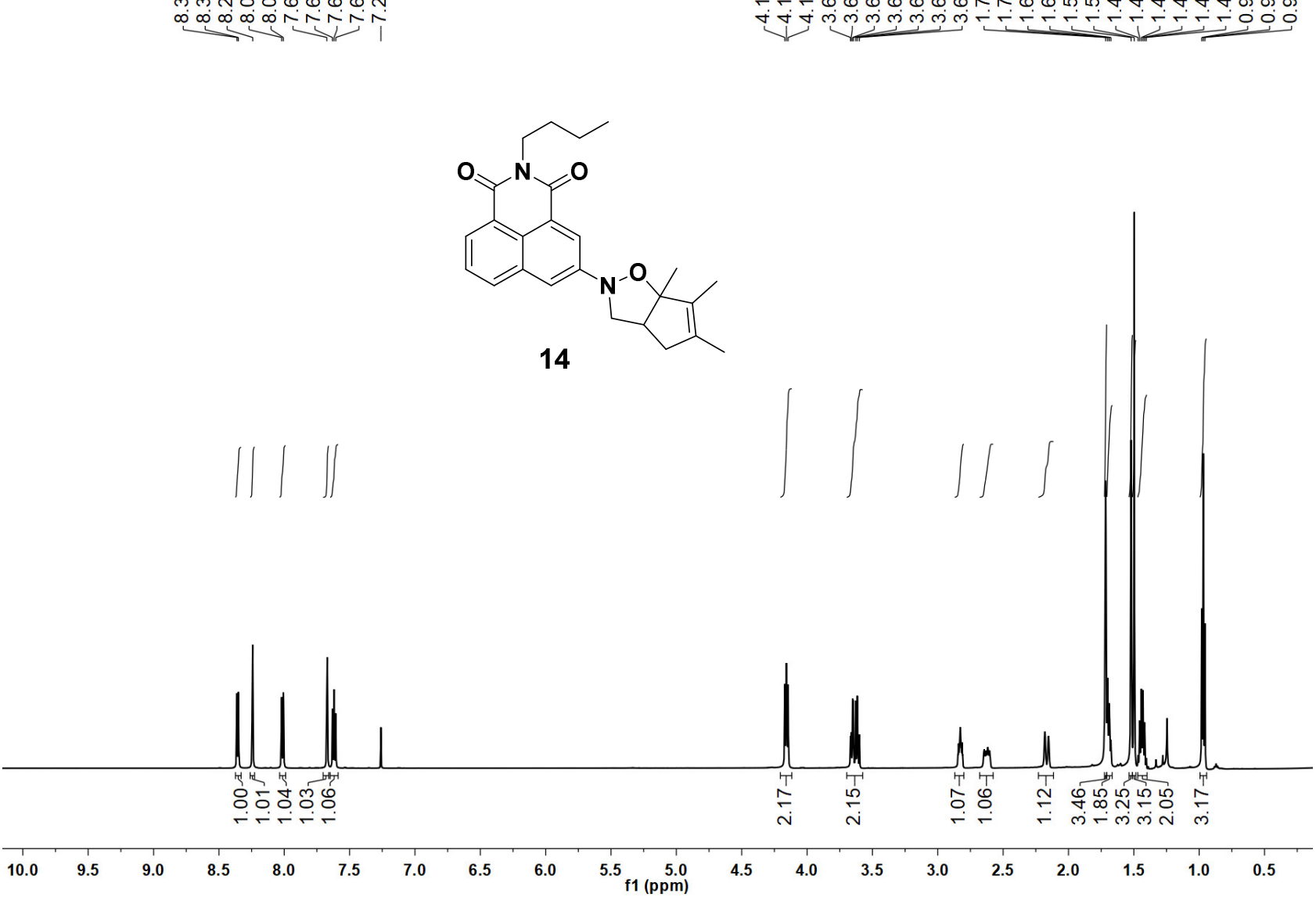

${ }^{13} \mathrm{C}$ NMR of compound $14\left(151 \mathrm{MHz}\right.$ in $\left.\mathrm{CDCl}_{3}\right)$

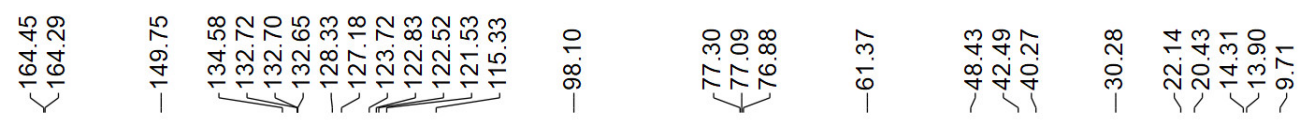
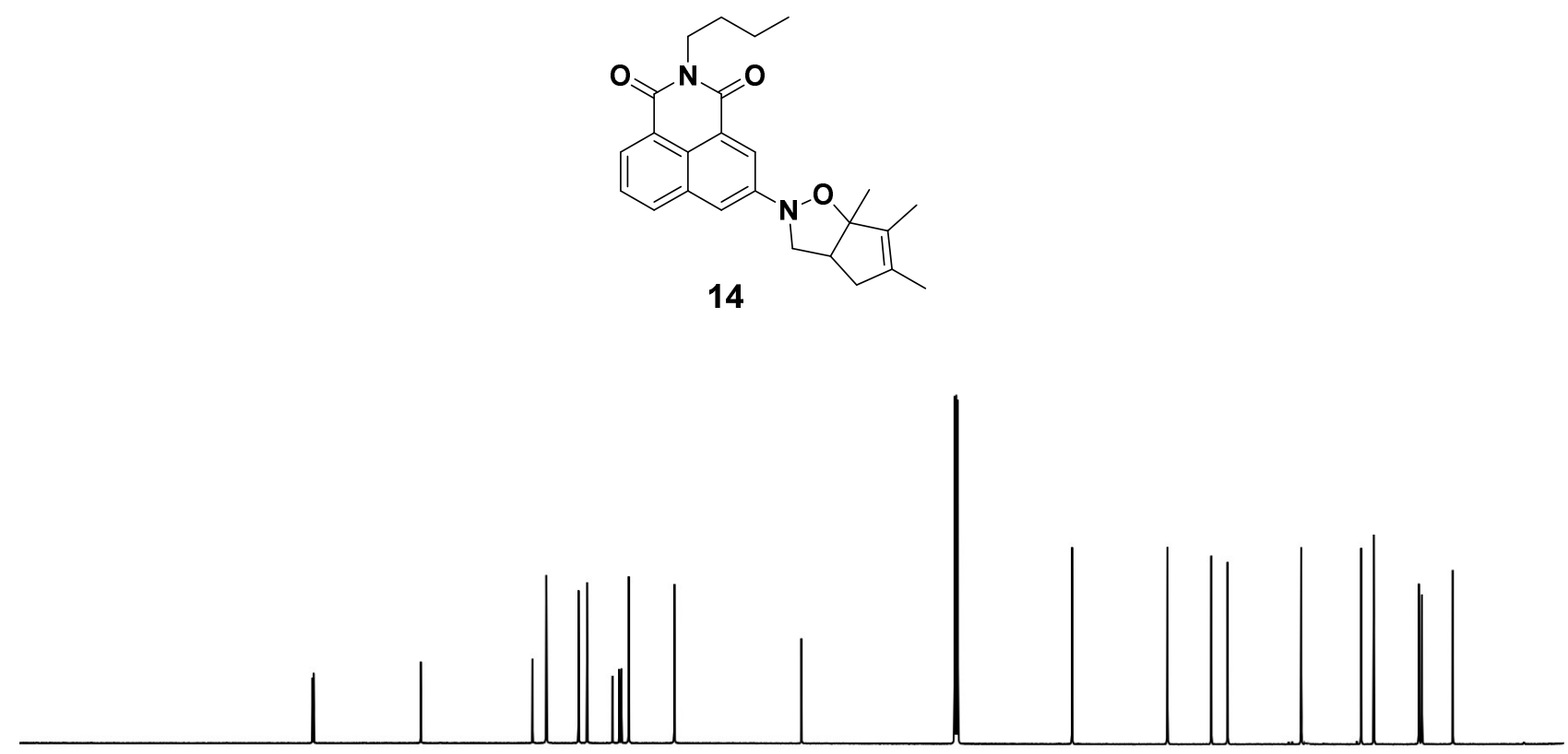

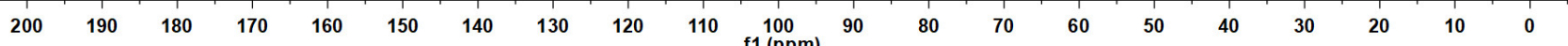


${ }^{1} \mathrm{H}$ NMR of compound $15\left(600 \mathrm{MHz}\right.$ in $\left.\mathrm{CD}_{3} \mathrm{CN}\right)$

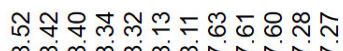

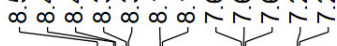<smiles>C=C(NC1=c2cccc3c2=C2C=CC=C1C3(OO)N(CCCC)C2=O)C1=C(C)CC(C(F)(F)F)=C1C</smiles>

15

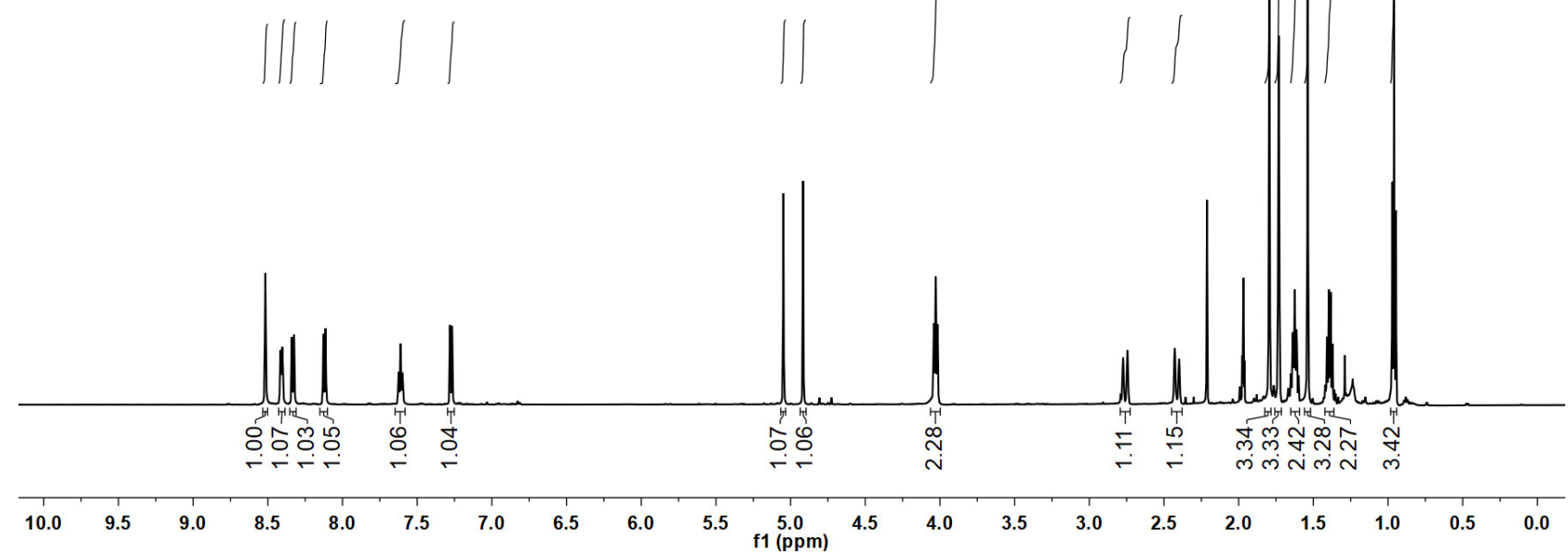

${ }^{13} \mathrm{C}$ NMR of compound 15 (151 MHz in $\left.\mathrm{CD}_{3} \mathrm{CN}\right)$

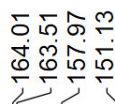

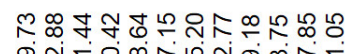

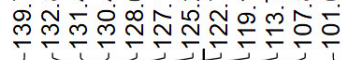

0
0
$\infty$
0

兽

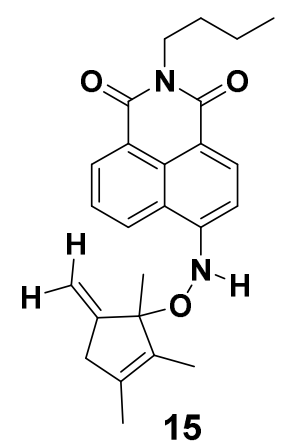


$\mathrm{H}-\mathrm{H}$ COSY of compound $15\left(600 \mathrm{MHz}\right.$ in $\left.\mathrm{CD}_{3} \mathrm{CN}\right)$

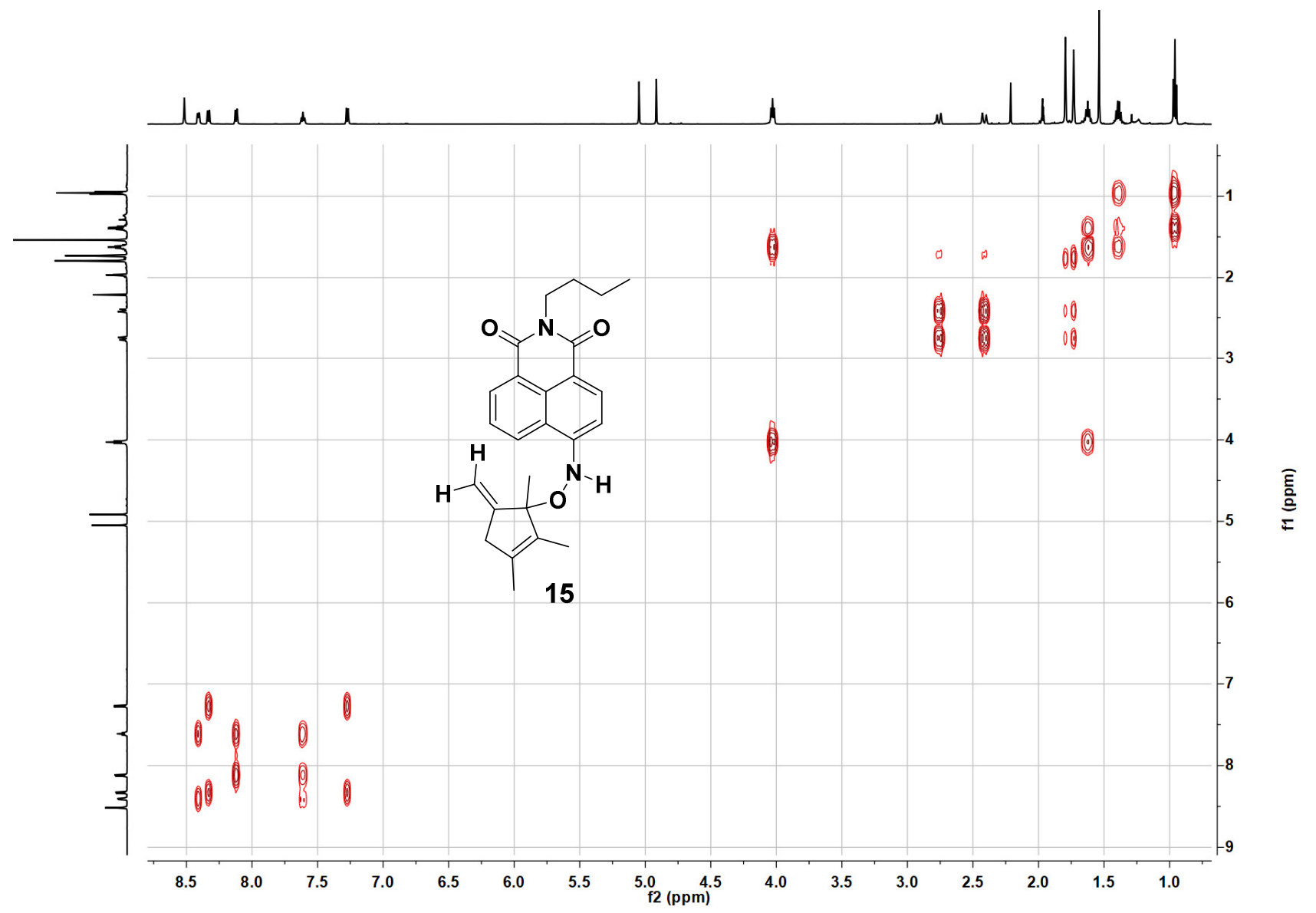

HSQC of compound $15\left(600 \mathrm{MHz}\right.$ in $\left.\mathrm{CD}_{3} \mathrm{CN}\right)$

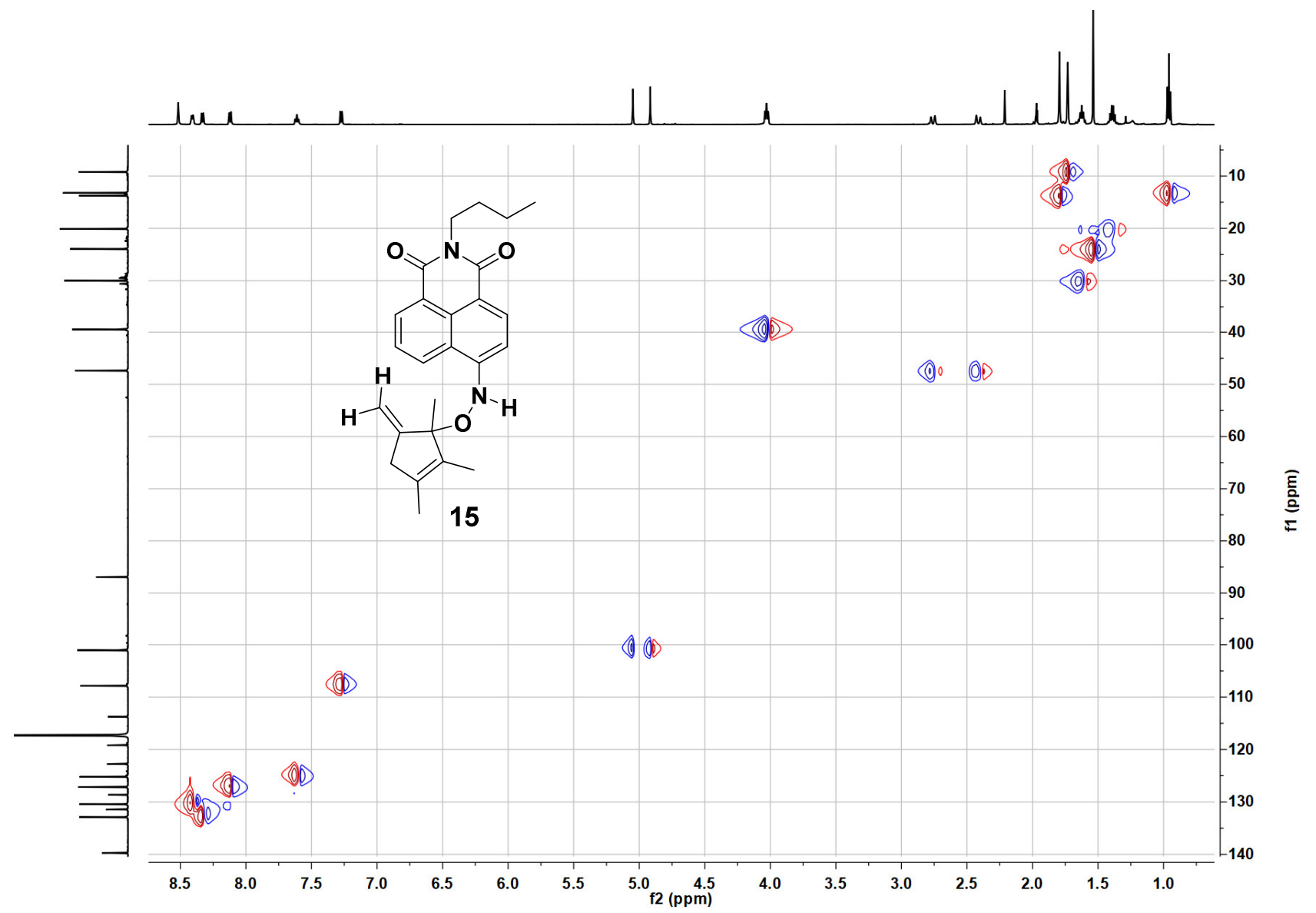


HMBC of compound $15\left(600 \mathrm{MHz}\right.$ in $\left.\mathrm{CD}_{3} \mathrm{CN}\right)$

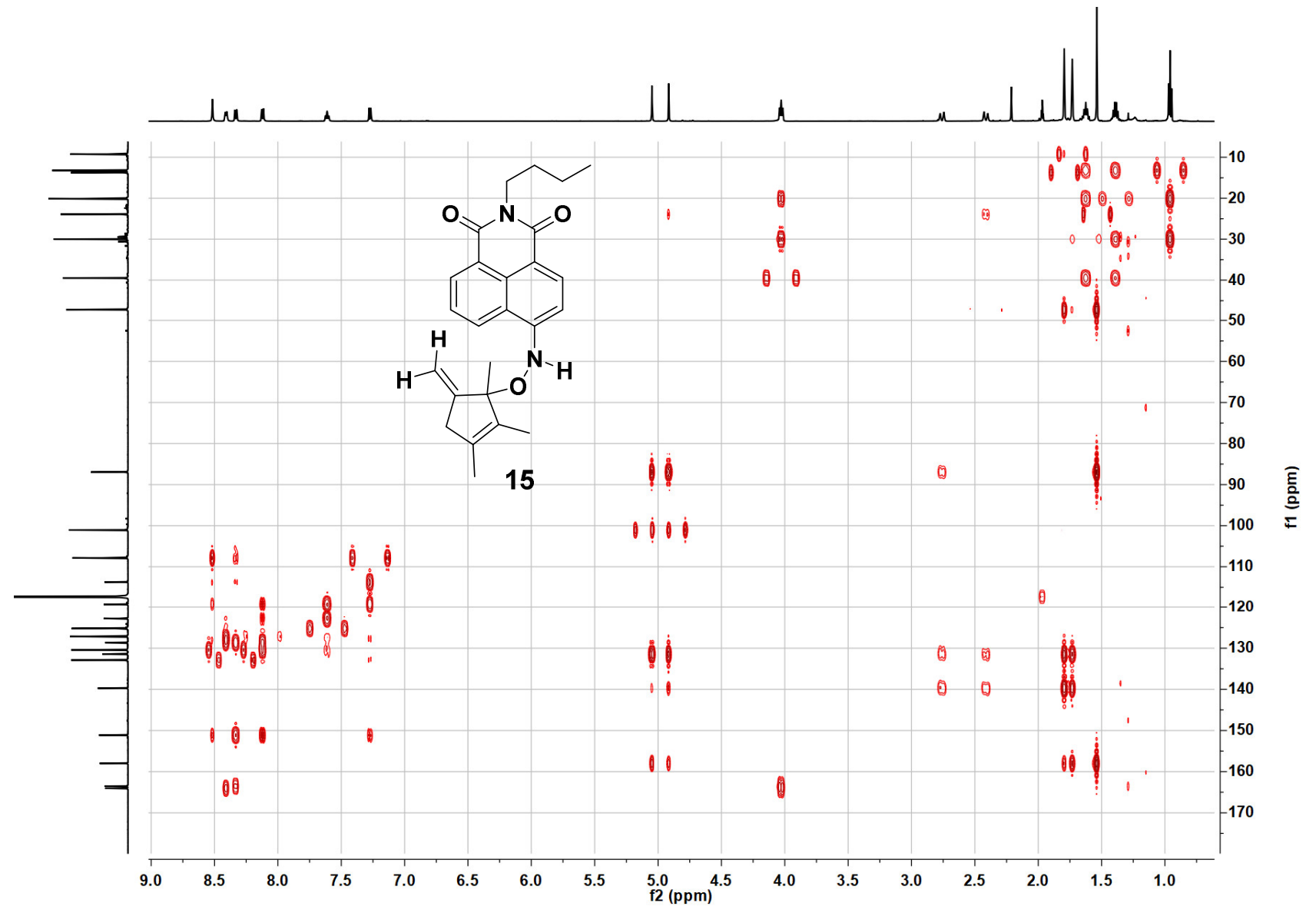


${ }^{1} \mathrm{H}$ NMR of compound $16\left(400 \mathrm{MHz}\right.$ in $\left.\mathrm{CDCl}_{3}\right)$

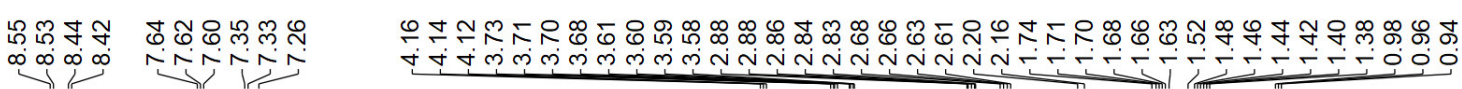

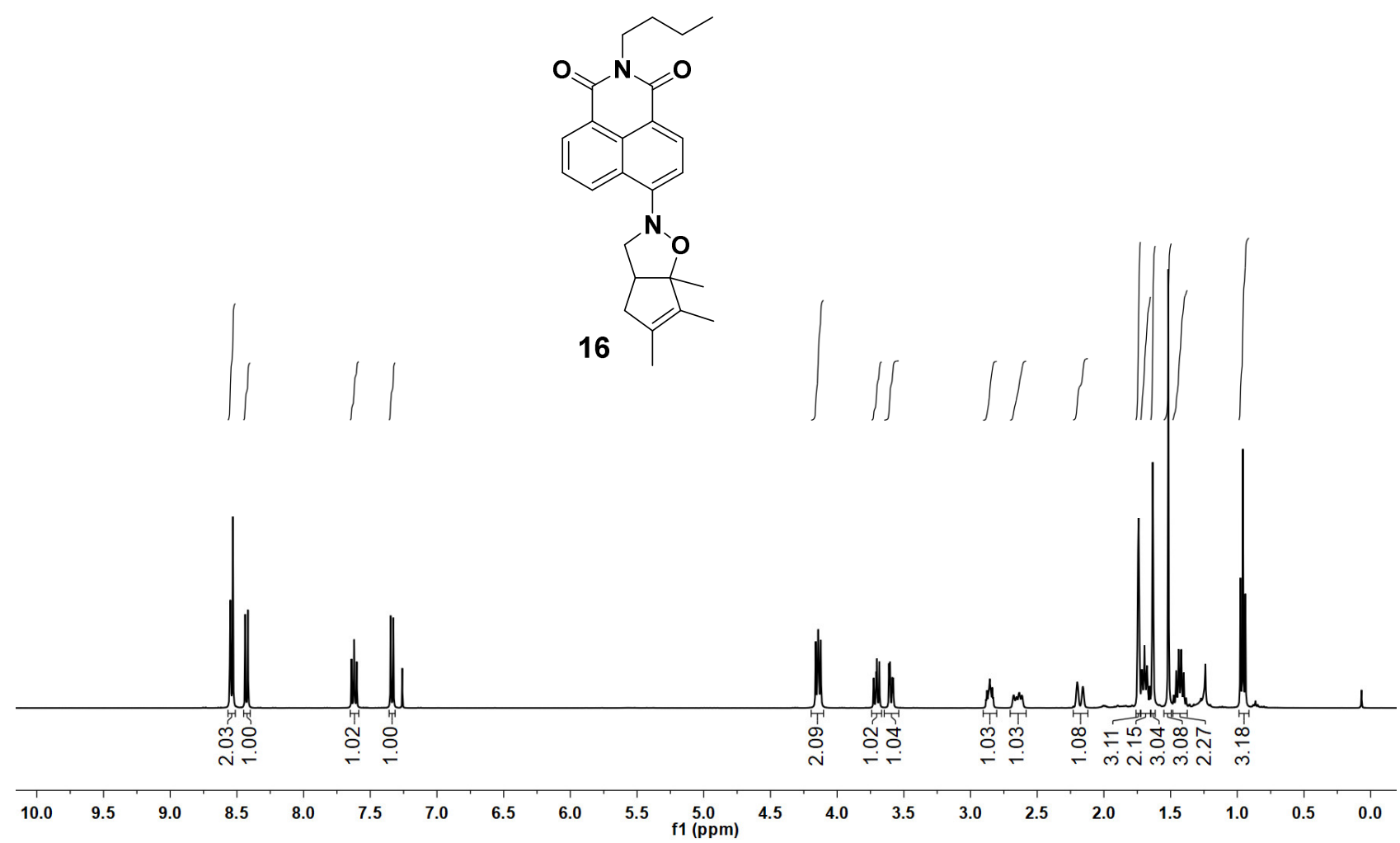

${ }^{13} \mathrm{C}$ NMR of compound $\mathbf{1 6}\left(101 \mathrm{MHz}\right.$ in $\left.\mathrm{CDCl}_{3}\right)$

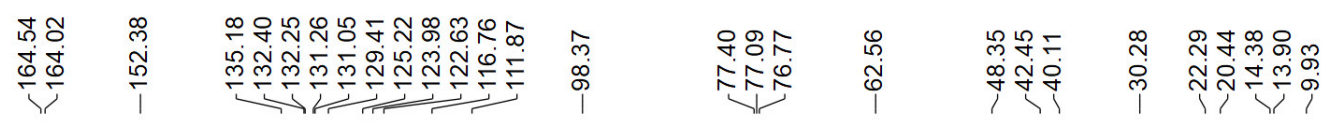

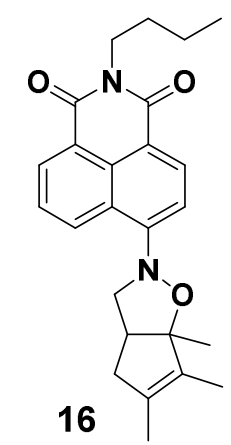

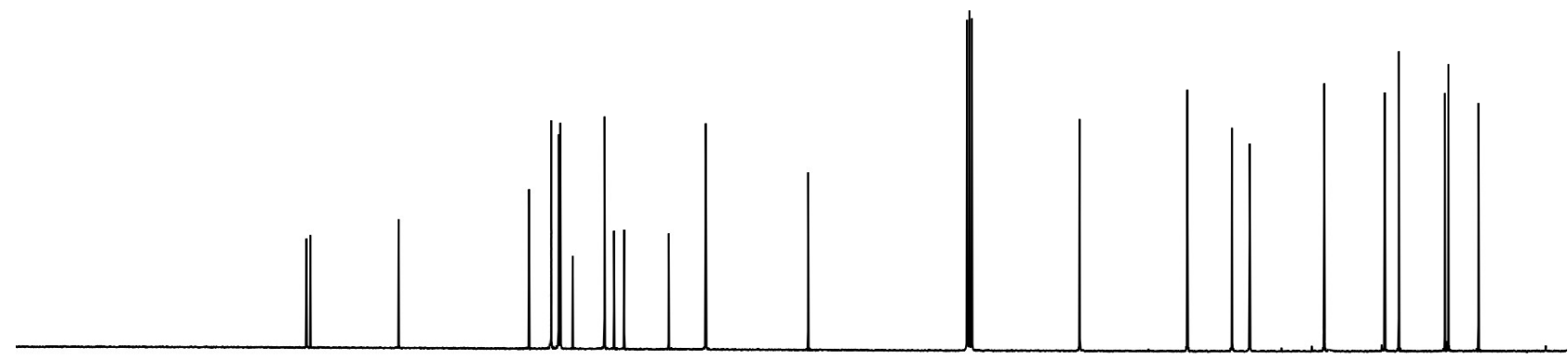


${ }^{1} \mathrm{H}$ NMR of compound $17\left(600 \mathrm{MHz}\right.$ in $\left.\mathrm{CD}_{3} \mathrm{CN}\right)$

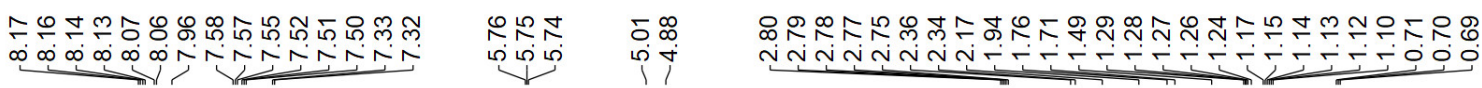

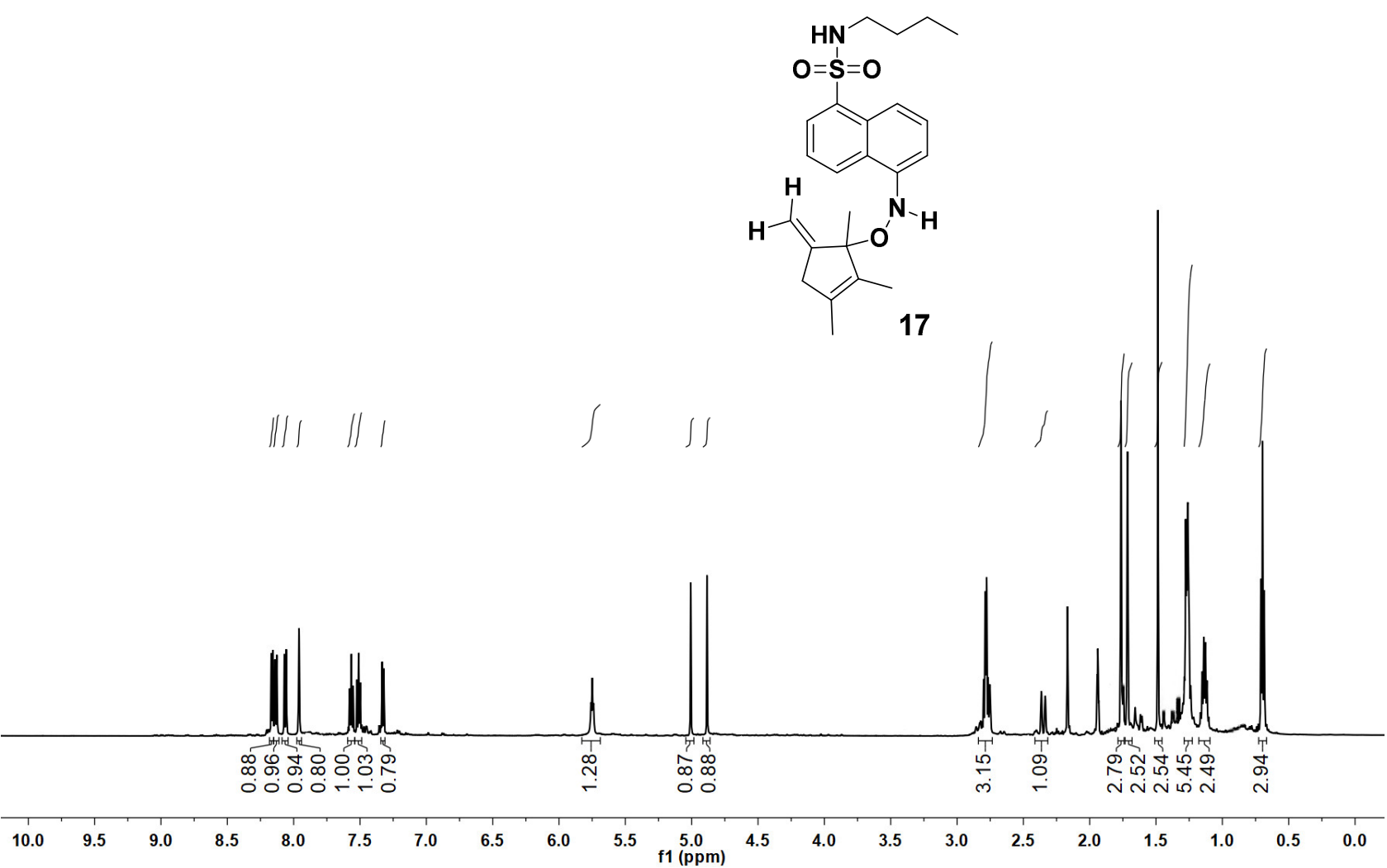

${ }^{13} \mathrm{C}$ NMR of compound $17\left(151 \mathrm{MHz}\right.$ in $\left.\mathrm{CD}_{3} \mathrm{CN}\right)$
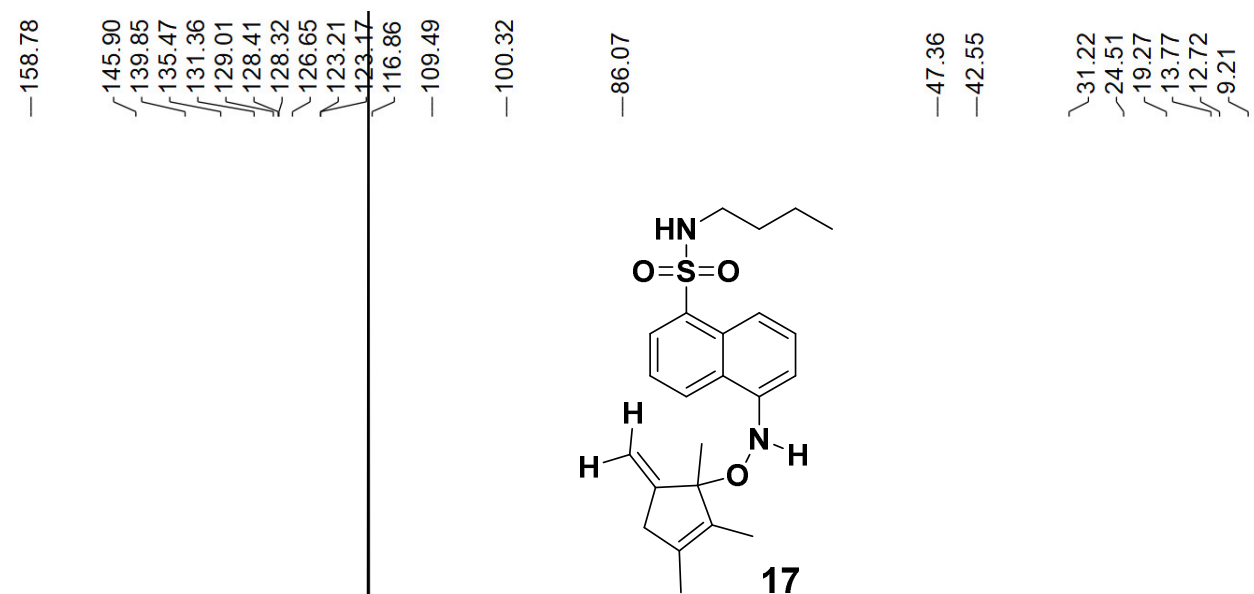

17

$200 \quad 190$

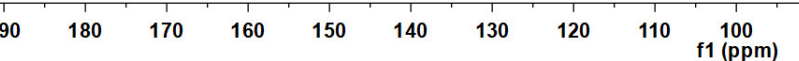


${ }^{1} \mathrm{H}$ NMR of compound Cyh-NHS (400 $\mathrm{MHz}$ in $\mathrm{CDCl}_{3}$ )

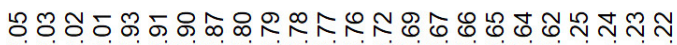

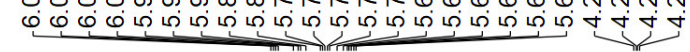

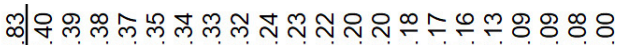<smiles>O=C(OCC1C=CC=CC1)ON1C(=O)CCC1=O</smiles>

Cyh-NHS

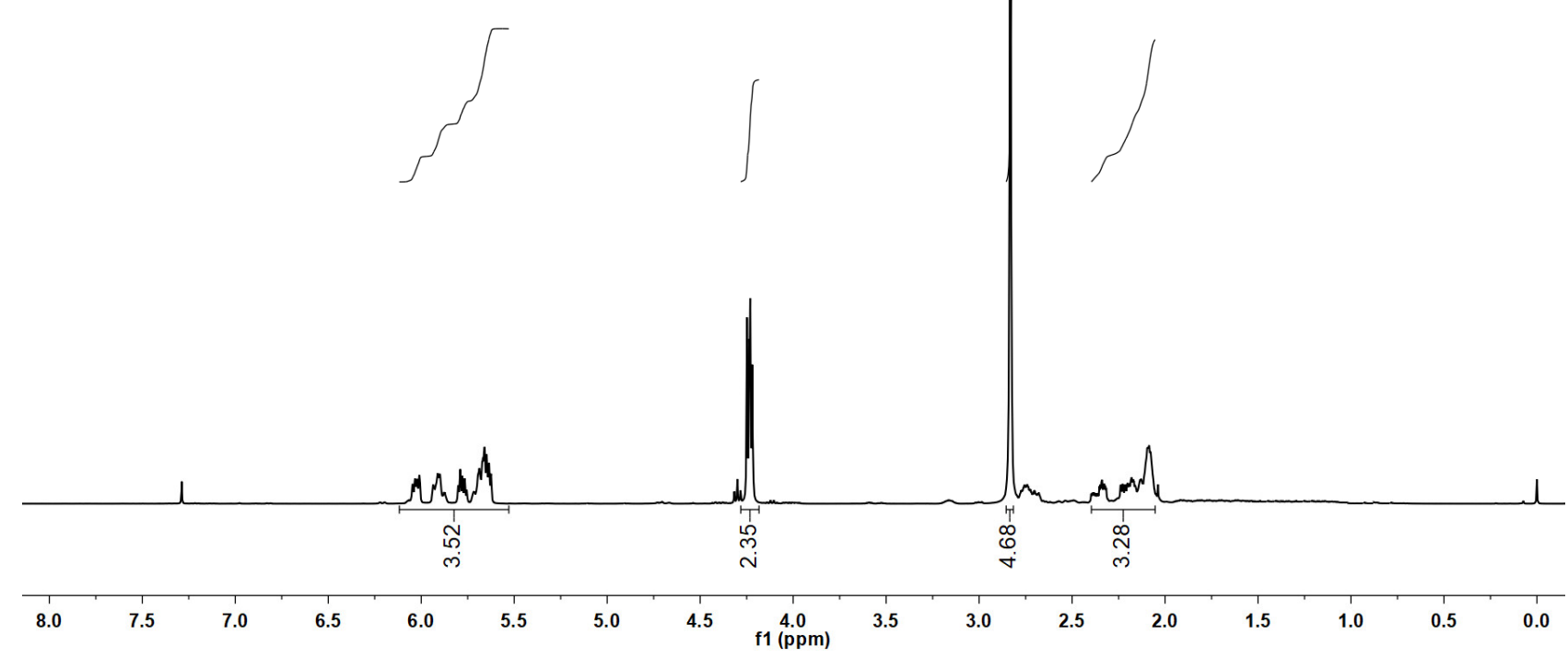

${ }^{13} \mathrm{C}$ NMR of compound Cyh-NHS (101 MHz in $\left.\mathrm{CDCl}_{3}\right)$

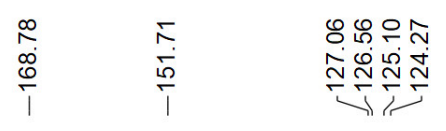

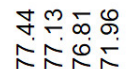

全少采

요요요 -

กึ่ำ<smiles>O=C(OCC1C=CC=CC1)ON1C(=O)CCC1=O</smiles>

Cyh-NHS

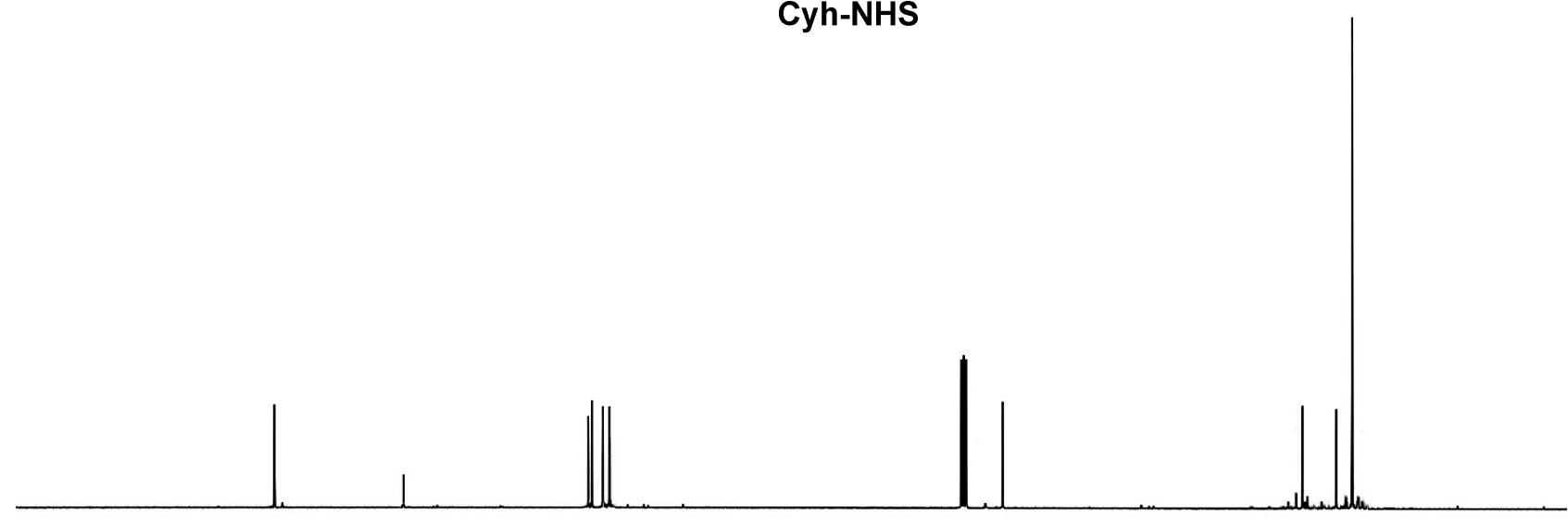

200

$190 \quad 180$

$180 \quad 170 \quad 160$

$\begin{array}{llll}150 & 140 & 130 & 120\end{array}$

110100

90 
${ }^{1} \mathrm{H}$ NMR of compound Cyh-TPP $\left(400 \mathrm{MHz}\right.$ in $\left.\mathrm{CD}_{3} \mathrm{OD}\right)$

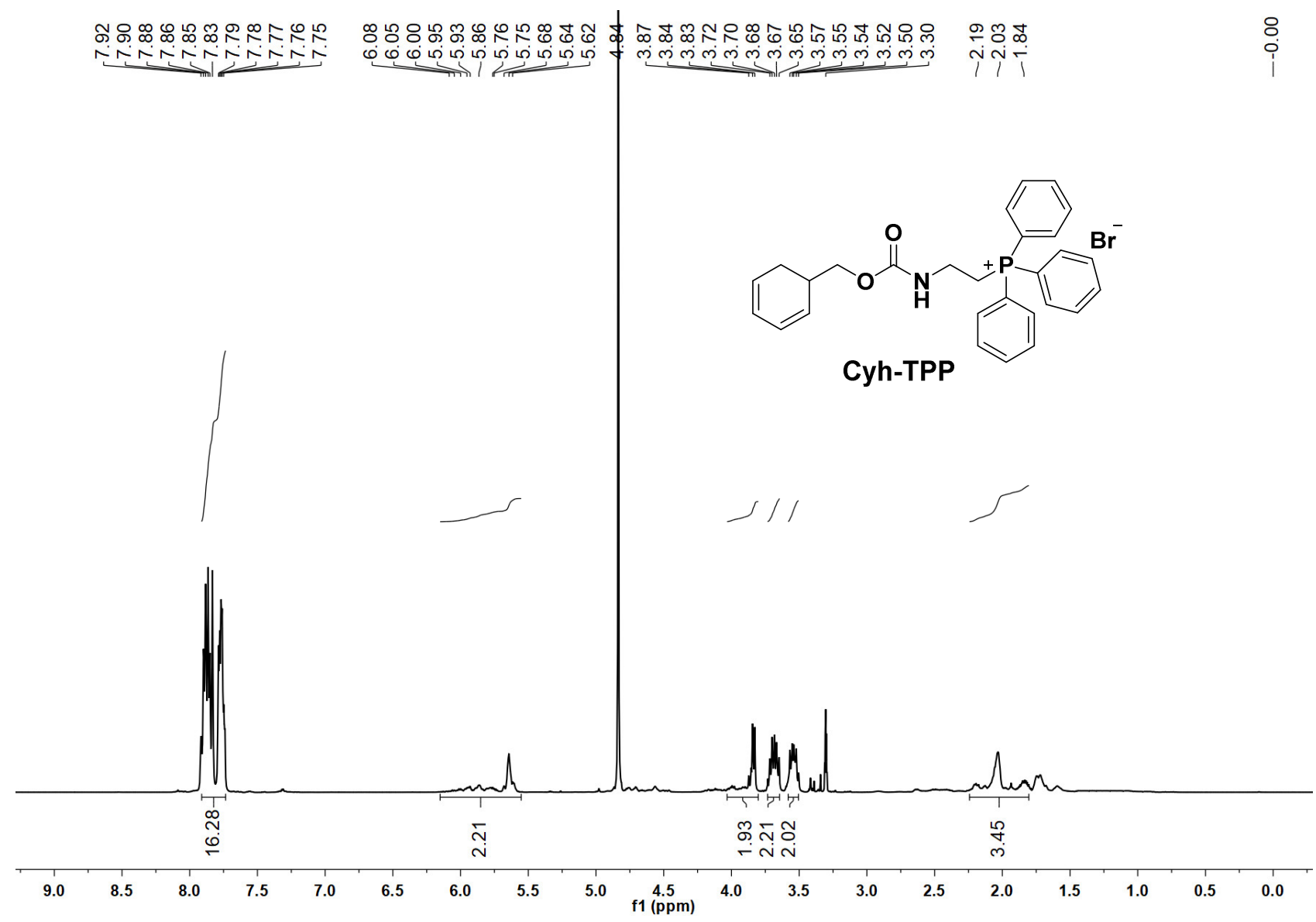

${ }^{13} \mathrm{C}$ NMR of compound Cyh-TPP $\left(101 \mathrm{MHz}\right.$ in $\left.\mathrm{CD}_{3} \mathrm{OD}\right)$

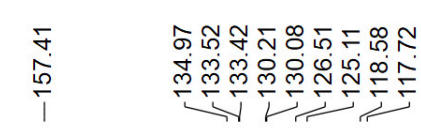<smiles>O=C(NCCP(c1ccccc1)(c1ccccc1)(c1ccccc1)c1ccccc1)OCC1C=CC=CC1</smiles>

Cyh-TPP

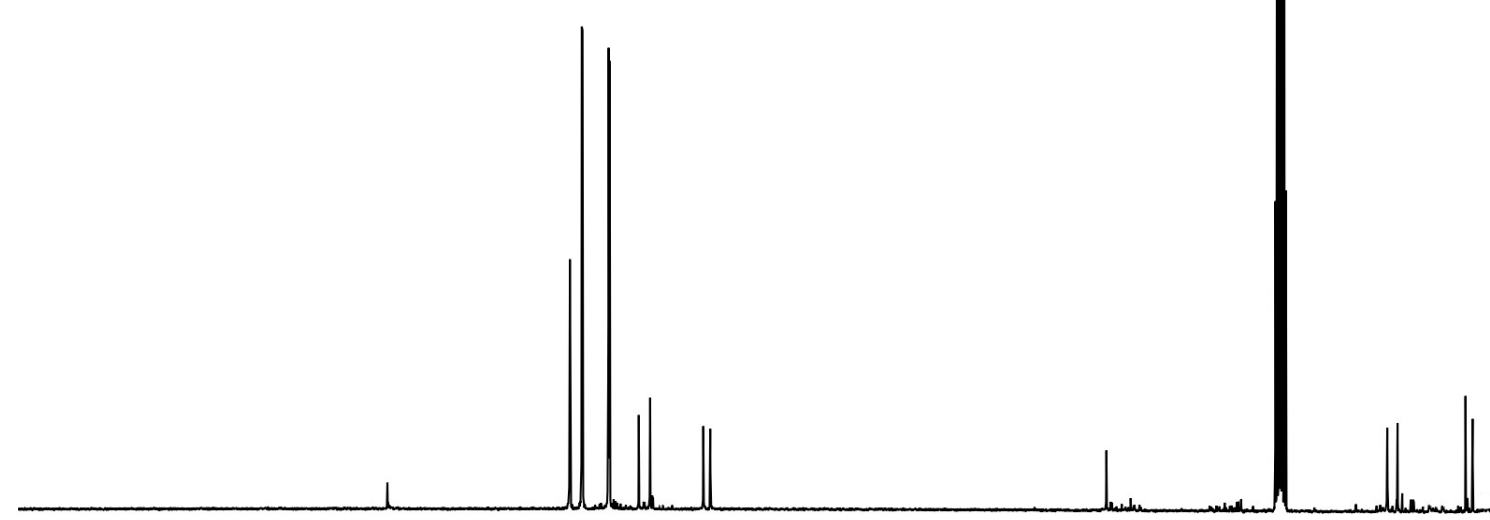


${ }^{1} \mathrm{H}$ NMR of compound $\mathbf{S 6}$ (400 MHz in $\mathrm{CDCl}_{3}$ )

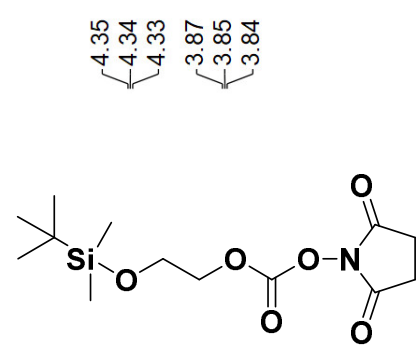

S6

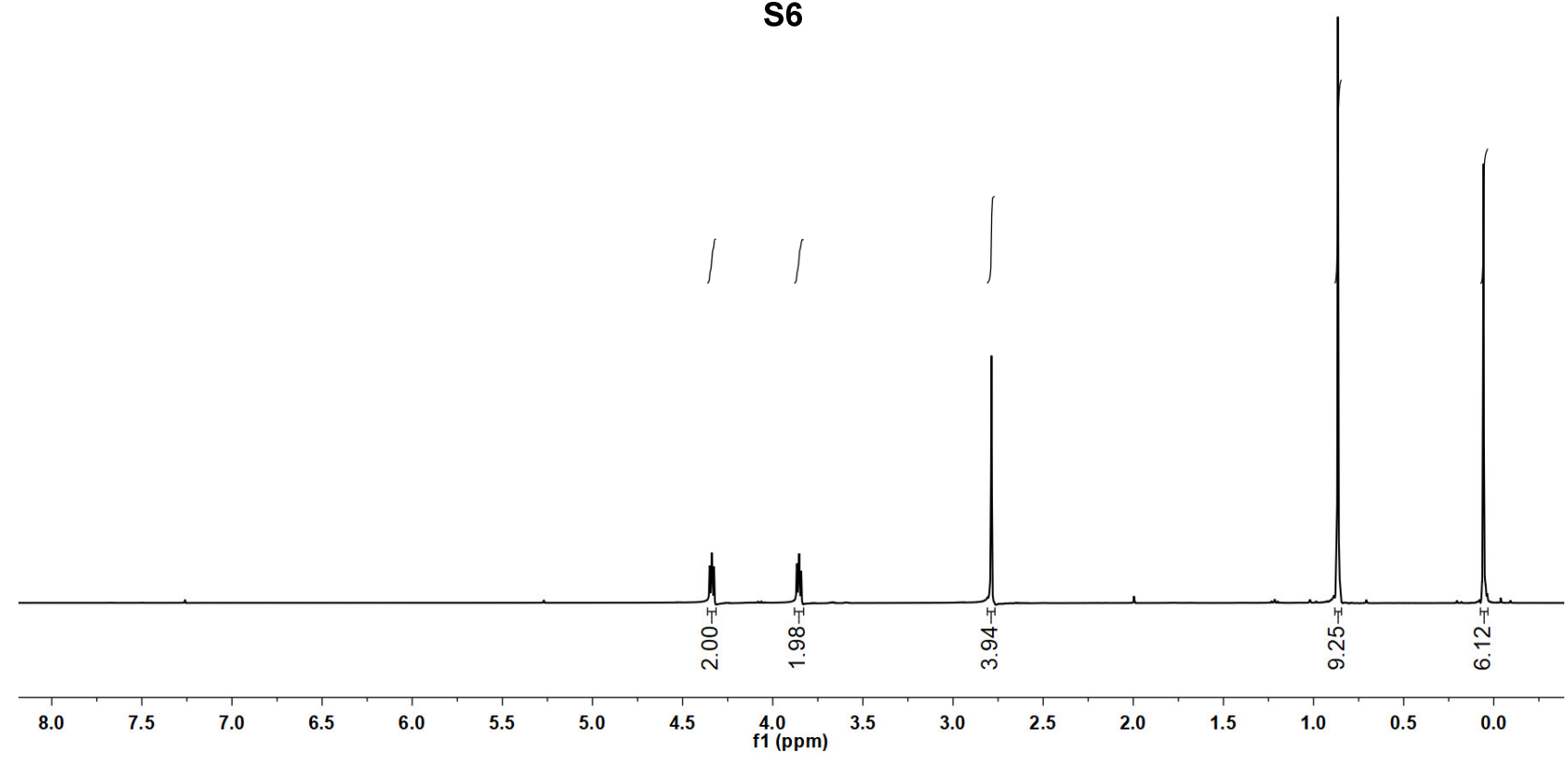

${ }^{13} \mathrm{C}$ NMR of compound $\mathbf{S 6}\left(101 \mathrm{MHz}\right.$ in $\left.\mathrm{CDCl}_{3}\right)$

$$
\begin{array}{ll}
\frac{1}{2} & 0 \\
0 & \frac{1}{5}
\end{array}
$$

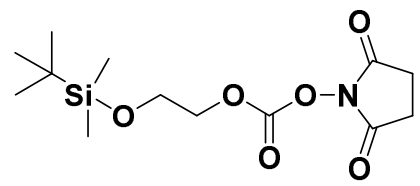

S6

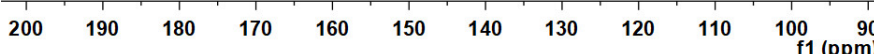


${ }^{1} \mathrm{H}$ NMR of compound $\mathbf{S} 7\left(400 \mathrm{MHz}\right.$ in $\left.\mathrm{CDCl}_{3}\right)$

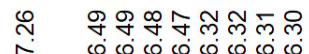

$$
\begin{aligned}
& \text { i }
\end{aligned}
$$

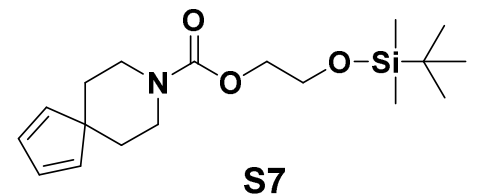

S7

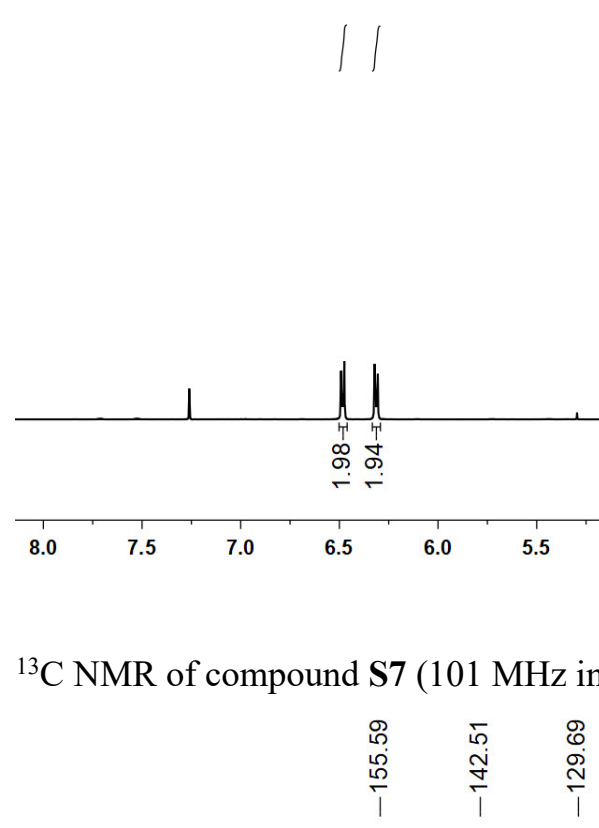

前先

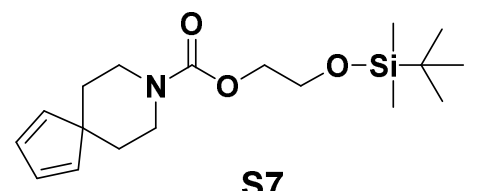

S7

$\begin{array}{lllllllllll}200 & 190 & 180 & 170 & 160 & 150 & 140 & 130 & 120 & 110 & 100 \\ \mathrm{f} 1(\mathrm{ppm}) & 90\end{array}$ 
${ }^{1} \mathrm{H}$ NMR of compound $\mathbf{S 8}\left(400 \mathrm{MHz}\right.$ in $\left.\mathrm{CDCl}_{3}\right)$<smiles>O=C(OCCO)N1CCC2(C=CC=C2)CC1</smiles>

\section{S8}
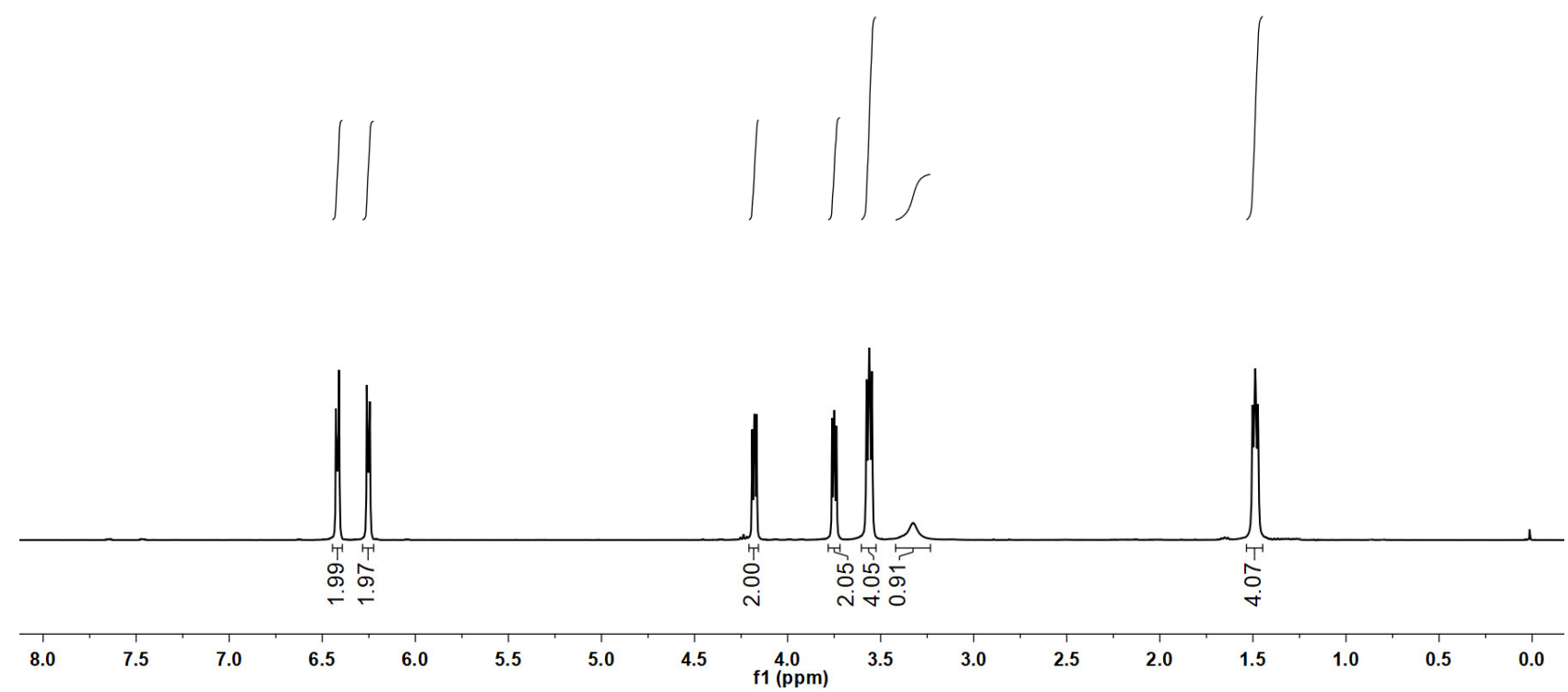

${ }^{13} \mathrm{C}$ NMR of compound $\mathbf{S 8}\left(101 \mathrm{MHz}\right.$ in $\left.\mathrm{CDCl}_{3}\right)$

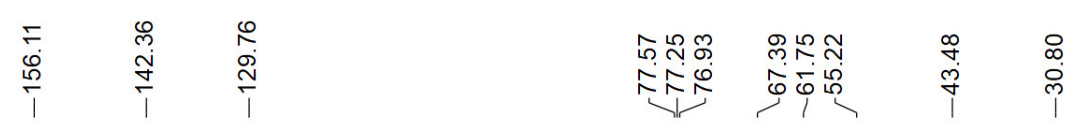

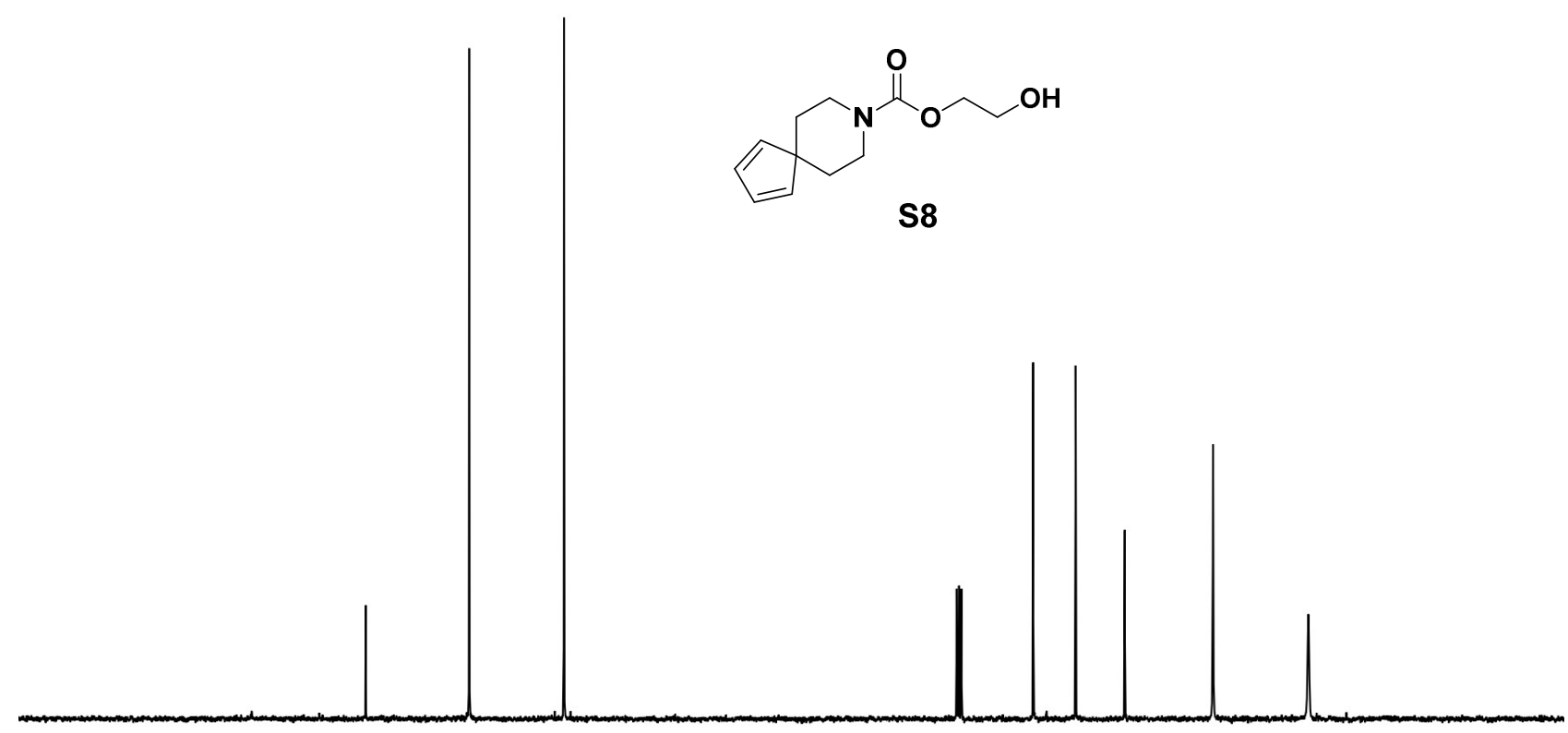


${ }^{1} \mathrm{H}$ NMR of compound Spd-NHS $\left(600 \mathrm{MHz}\right.$ in $\left.\mathrm{CDCl}_{3}\right)$

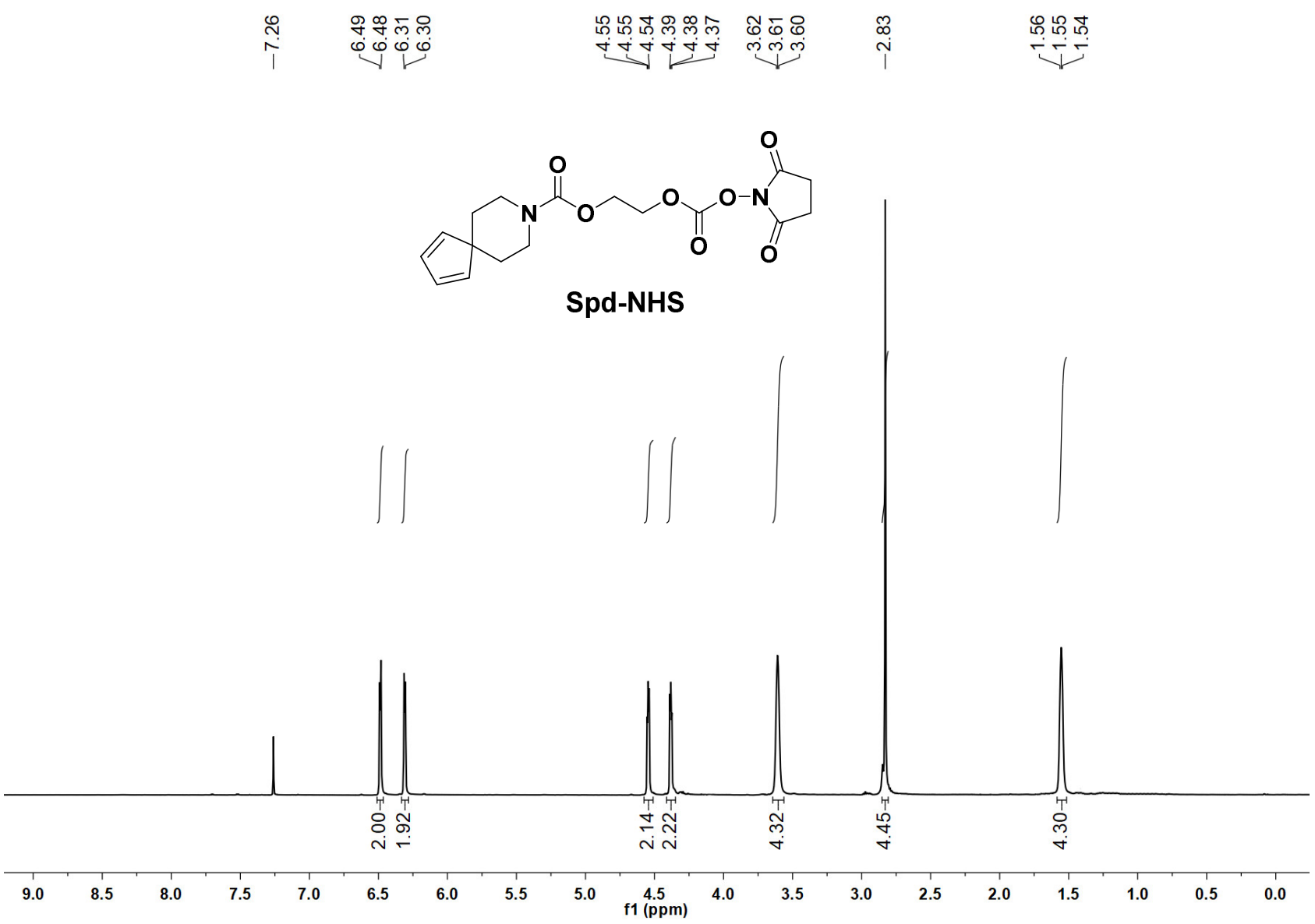

${ }^{13} \mathrm{C}$ NMR of compound Spd-NHS (151 MHz in $\left.\mathrm{CDCl}_{3}\right)$

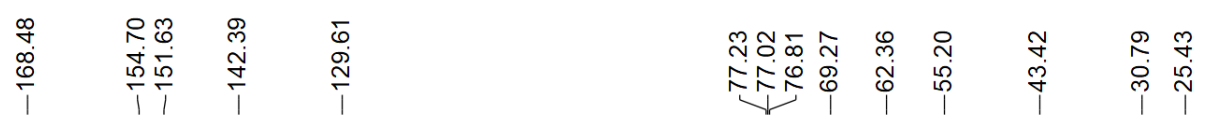<smiles>O=C(OCCOC(=O)N1CCC2(C=CC=C2)CC1)ON1C(=O)CCC1=O</smiles>

Spd-NHS

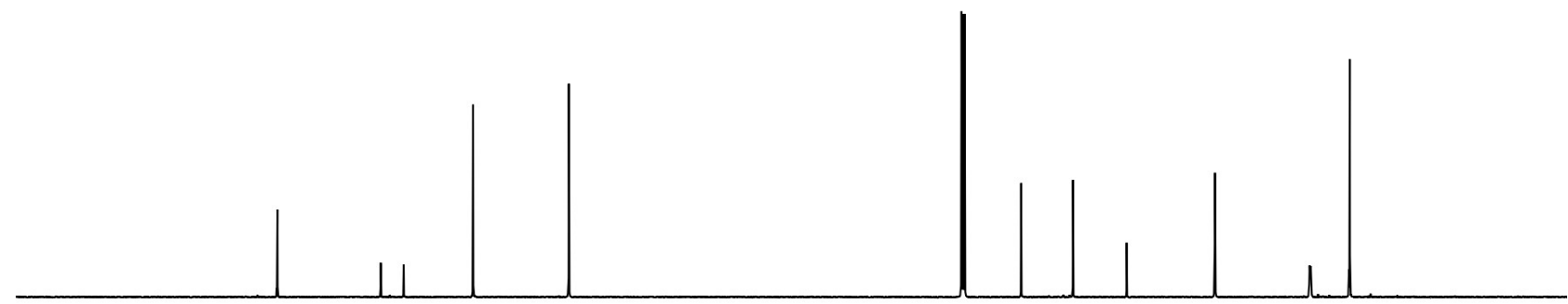

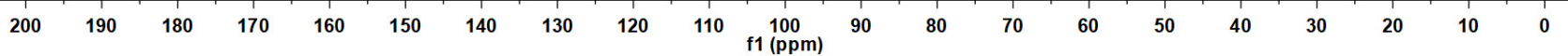


${ }^{1} \mathrm{H}$ NMR of compound Mcp-NHS (400 MHz in $\mathrm{CDCl}_{3}$ )

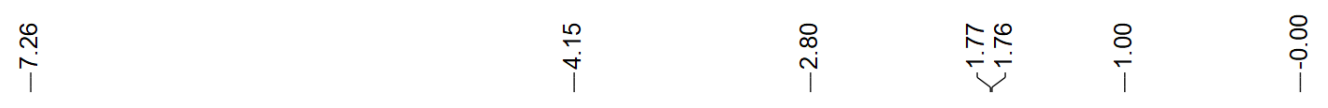

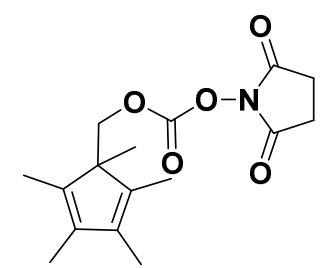

Mcp-NHS

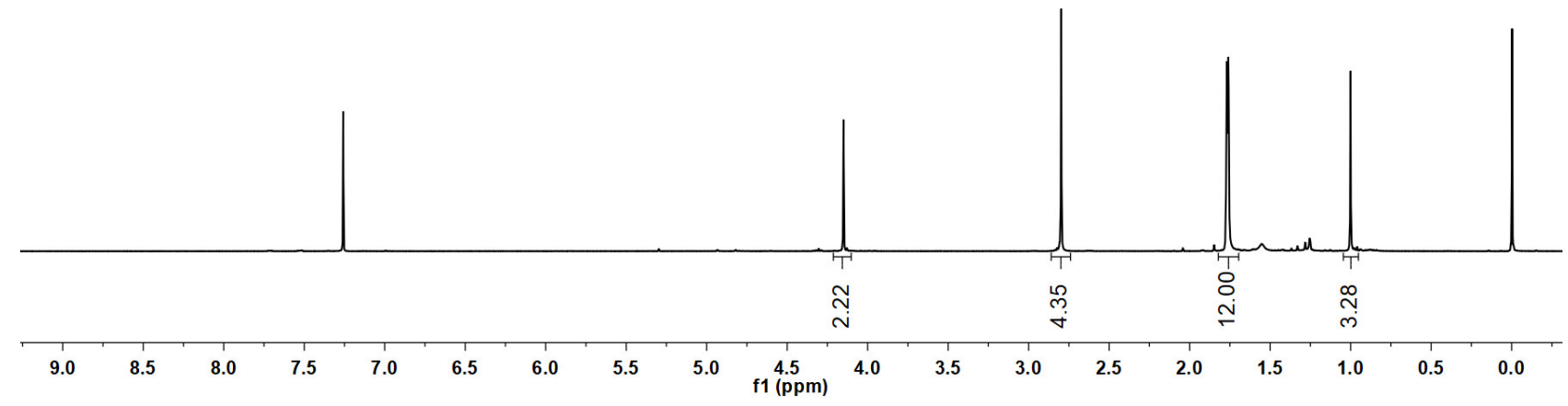

${ }^{13} \mathrm{C}$ NMR of compound Mcp-NHS $\left(151 \mathrm{MHz}\right.$ in $\left.\mathrm{CDCl}_{3}\right)$

点

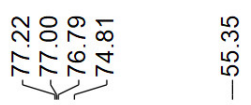

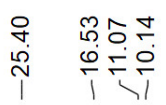

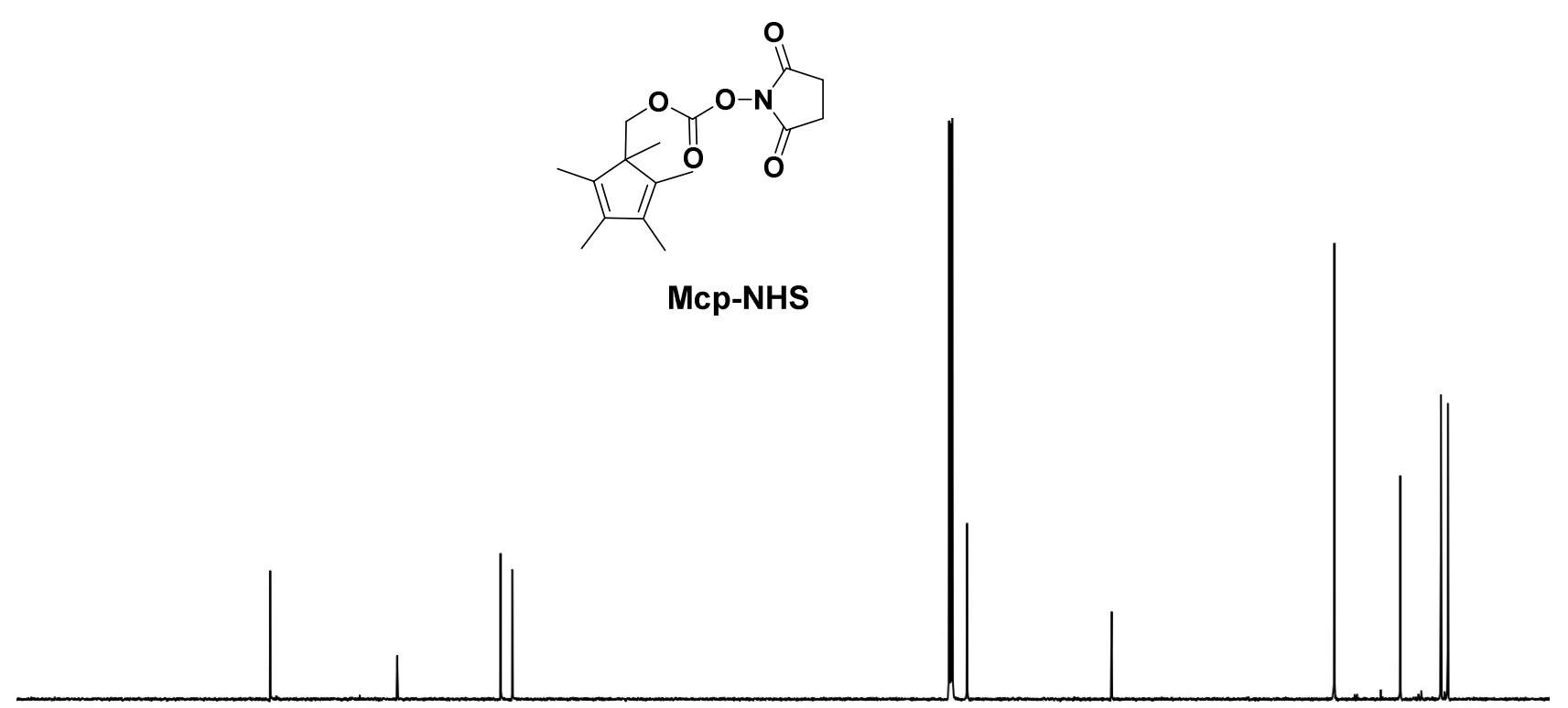

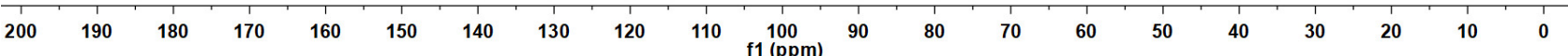


${ }^{1} \mathrm{H}$ NMR of compound Mcp-TPP (400 MHz in $\mathrm{CD}_{3} \mathrm{OD}$ )

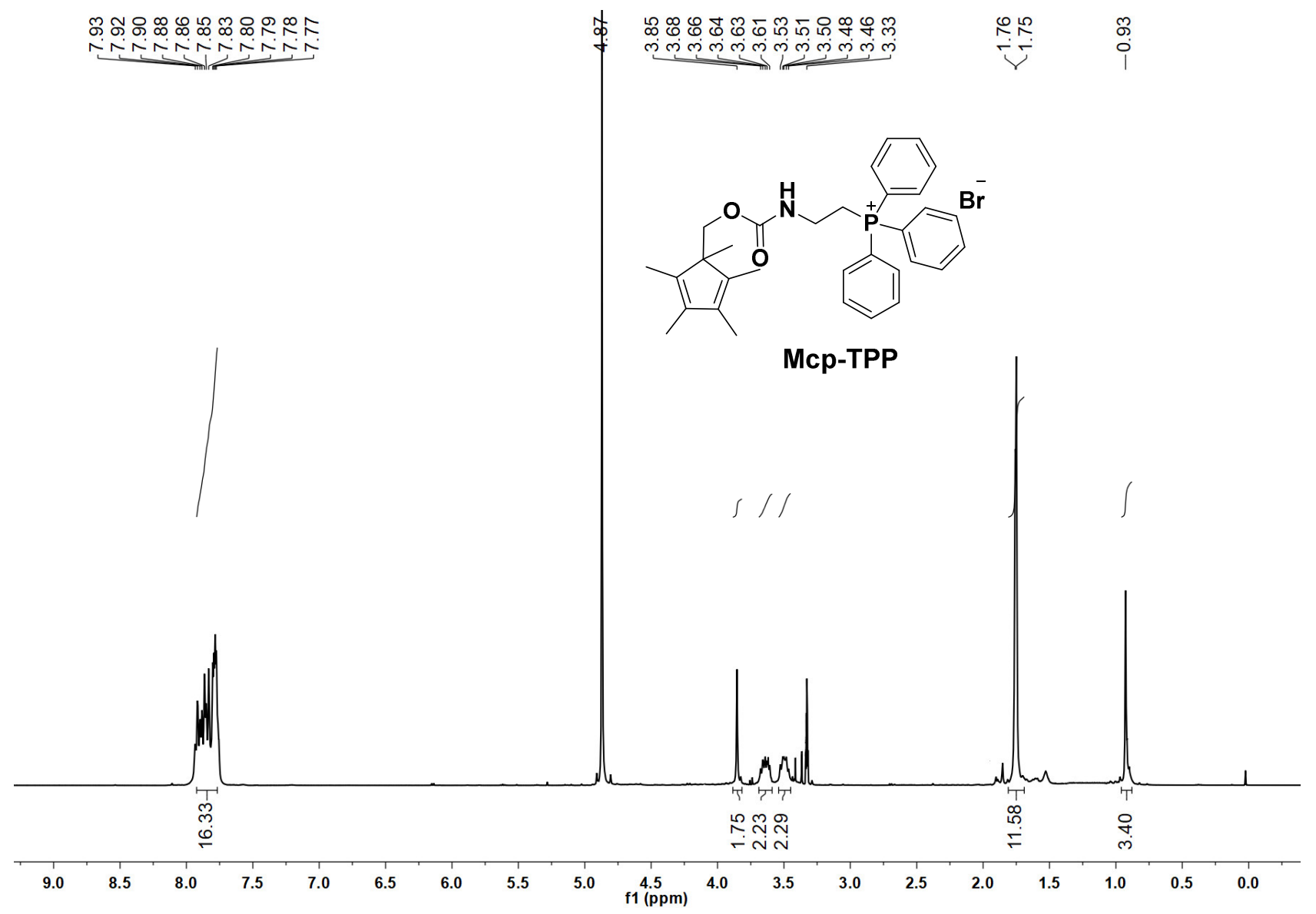

${ }^{13} \mathrm{C}$ NMR of compound Mcp-TPP $\left(101 \mathrm{MHz}\right.$ in $\left.\mathrm{CD}_{3} \mathrm{OD}\right)$
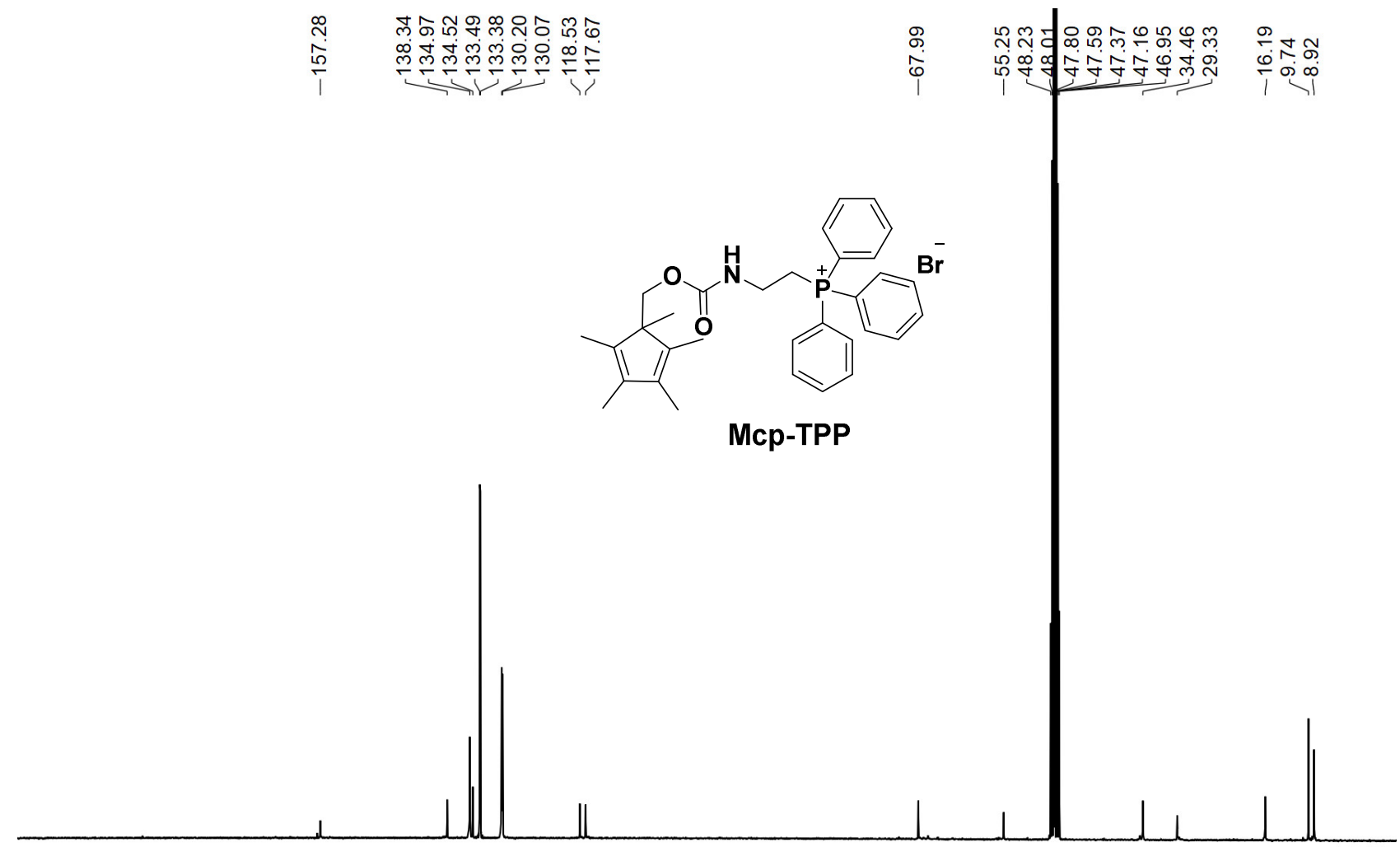

20

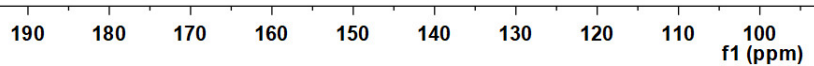

